\title{
Local Equilibrium States in Quantum Field Theory in Curved Spacetime
}

\author{
Dissertation \\ zur Erlangung des mathematisch-naturwissenschaftlichen Doktorgrades \\ „Doctor rerum naturalium” \\ der Georg-August-Universität zu Göttingen
}

vorgelegt von

Christoph Solveen

aus Bernburg (Saale)

Göttingen, 2012 
Referent: Prof. Dr. Karl-Henning Rehren Koreferent: Prof. Dr. Detlev Buchholz

Tag der mündlichen Prüfung: 11. April 2012 


\section{Zusammenfassung}

In der vorliegenden Arbeit wird der konzeptionellen Frage nachgegangen, unter welchen Bedingungen Zuständen in der Quantenfeldtheorie auf gekrümmten Raumzeiten thermische Parameter zugewiesen werden können. Der Untersuchung dieses Problems wird das Konzept des lokalen Gleichgewichts im Sinne von Buchholz, Ojima und Roos zugrundegelegt. In diesem Zugang werden punktweise lokalisierte Quantenfelder - sogenannte thermische Observable - verwendet, um makroskopischen Systemen, die lokal nicht zu weit vom thermischen Gleichgewicht abweichen, thermische Parameter zuzuordnen. Im Gegensatz zu den meisten existierenden Ansätzen erlaubt dies eine konzeptionell klare Beschreibung von Nichtgleichgewichtsphänomenen, wie sie z.B. in der Kosmologie diskutiert werden. Zur Illustration der Resultate wird das Beispiel des masselosen, konform gekoppelten, freien Skalarfeldes herangezogen.

Es wird zunächst die Struktur der Menge der thermischen Observablen untersucht. Es zeigt sich, dass diese in gekrümmter Raumzeit keinen Vektorraum bildet und als linear unabhängig gewählt werden muss. Zwischen den entsprechenden lokalen thermischen Parametern können allerdings Relationen auftreten, die durch lineare Zustandsgleichungen hervorgerufen werden. Es wird gezeigt, dass diese Beziehungen in gekrümmter Raumzeit zu Evolutionsgleichungen für die lokalen thermischen Parameter führen.

$\mathrm{Zu}$ thermischen Observablen, für die keine solchen Relationen bestehen, existieren in der Minkowskiraumzeit Zustände, denen in beschränkten Raumzeitgebieten die entsprechenden lokalen thermischen Parameter zugeordnet werden können. Ferner existieren unter einer natürlichen Annahme an das Spektrum der thermischen Observablen Zustände, die sich an jedem gegebenen Punkt einer gekrümmten Raumzeit im Gleichgewicht befinden.

Weiterhin wird gezeigt, dass KMS Zuständen in stationären gekrümmten Raumzeiten nicht diesselbe Bedeutung zukommt wie den globalen Gleichgewichtszuständen in der Minkowskiraumzeit. Der KMS Parameter $\beta$ kann nicht notwendigerweise als eine inverse Temperatur interpretiert werden, sondern setzt sich aus Beiträgen der Krümmung, der Beschleunigung des Beobachters und der lokalen Temperatur zusammen. Die bei dieser Untersuchung verwendeten thermischen Observablen weisen Renormierungsfreiheiten auf, die durch die Messungen in den KMS Zuständen durch physikalisch interpretierbare Parameter fixiert werden.

\section{Abstract}

The present work concerns itself with the conceptual problem of assigning thermal parameters to states in quantum field theory in curved spacetime, and is based on the concept of local thermal equilibrium in the sense of Buchholz, Ojima and Roos. In this approach, point-like localized quantum fields, so called thermal observables, are used to attach thermal parameters to macroscopic systems, with the proviso that these systems do not deviate too far from thermal equilibrium. In contrast to many of the existing approaches, this allows for a conceptually clear description of non-equilibrium phenomena, for example in cosmology. The results are illustrated using the massless, conformally coupled, free scalar field.

Initially, the structure of the set of thermal observables is discussed and it is found that in curved spacetime these objects do not form a vector space and must be chosen as linearly independent. However, there can be relations between the corresponding local thermal parameters which are induced by linear equations of state. It is shown that these relations lead to evolution equations for the local thermal parameters in curved spacetime.

For thermal observables where no such linear relations hold, there exist states to which the corresponding local thermal parameters can be assigned in bounded regions in Minkowski spacetime. Using a natural assumption regarding the spectrum of the thermal observables, it is shown that states exist which are in equilibrium at any given point in a curved spacetime.

Following on, it is argued that KMS states in stationary curved spacetimes may not be viewed 
in analogy to global thermal equilibrium states in Minkowski spacetime. The KMS parameter $\beta$ cannot necessarily be interpreted as an inverse temperature, but consists of contributions from the curvature, the acceleration of the observer, and the local temperature. The thermal observables used in this investigation exhibit some renormalization freedom, which can be fixed in terms of physically meaningful parameters. 


\section{Contents}

\begin{tabular}{lll}
\hline 0 & Introduction & 1
\end{tabular}

\begin{tabular}{|lll}
1 & Quantum Field Theory in Curved Spacetime & 7
\end{tabular}

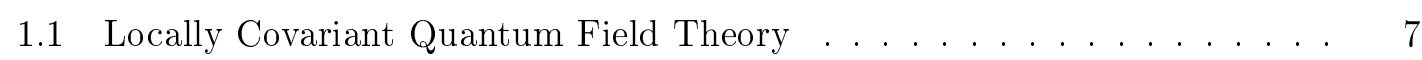

1.1 .1 Spacetime Geometry . . . . . . . . . . . . . . . 8

1.1 .2 Observables and General Covariance . . . . . . . . . . . . . . 11

1.1 .3 States . . . . . . . . . . . . . . . . . . . . . 14

1.2 The Free Scalar Field and Wick Polynomials. . . . . . . . . . . . . . . 16

$1.2 .1 \quad$ The Klein Gordon Equation and Quantization. . . . . . . . . . . . 16

1.2.2 Hadamard States and the Extended Algebra of Observables . . . . 20

1.2 .3 Locally Covariant Wick Polynomials . . . . . . . . . . . . . 26

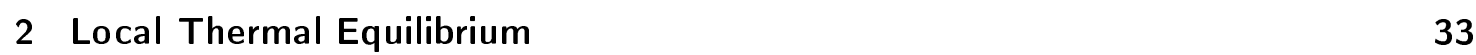

2.1 Local Thermal Equilibrium States in Minkowski Spacetime . . . . . . . . 33

$2.1 .1 \quad$ Basic Definitions . . . . . . . . . . . . . . . . 33

$2.1 .2 \quad$ Existence of Local Thermal Equilibrium States . . . . . . . . . . . 37

2.2 Local Thermal Equilibrium States in Curved Spacetime . . . . . . . . . . 40

2.2 .1 Definition of Local Equilibrium in Curved Backgrounds . . . . . . 40

$2.2 .2 \quad$ Existence of Local Equilibrium in Curved Backgrounds . . . . . . . 46

$2.2 .3 \quad$ Unboundedness and Scaling Limits . . . . . . . . . . . . . . . . 49

3 Local Thermal Equilibrium States for the Free Scalar Field $\quad 55$

3.1 Basic Thermal Observables and Thermal Functions . . . . . . . . . . . . 55

3.2 Local Thermal Equilibrium and KMS States in Stationary Spacetimes . . 67

4 Conclusion \& Perspectives 83

\begin{tabular}{lr}
\hline Bibliography & 92
\end{tabular}

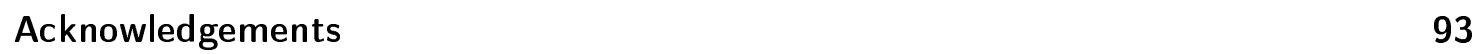




\section{Introduction}

In the description of macroscopic physical systems, one often finds the term "local temperature", meaning that there is a spacetime dependent parameter that somehow takes the rôle of temperature in systems which are not everywhere and at any time (i.e. globally) in equilibrium. However, it is only for systems in global equilibrium where thermostatic quantities like temperature are well-defined. While global equilibrium is of course an idealization and local temperature is an important tool in physics [ZMR96], the conceptual basis for the notion of local equilibrium is often quite unclear.

In the context of quantum field theory (QFT) in Minkowski spacetime, Buchholz, Ojima and Roos have presented a way to define states which can be considered to be in local thermal equilibrium (LTE) [BOR02]. One of several promising features of this method is that thermal parameters like temperature are attached to these states, depending on their microscopic properties, in a conceptually clear and mathematically precise manner. Since the definition is purely local, Buchholz and Schlemmer were subsequently able to give a generalization of LTE states to QFT in curved spacetime [BS07].

This is quite important; while the notion of LTE is relevant already in flat spacetime, it should be an indispensable tool in the description of macroscopic systems in the presence of spacetime curvature, which, in general, prevents any system from being in global equilibrium. Cosmological models are a good illustration of this point: being non-stationary spacetimes they do not allow strict global equilibrium and one has to resort to local concepts. As a consequence, one often finds intensive macroscopic observables like temperature, entropy densities and pressure which are supposed to vary in cosmological time (and in space).

For example, the cosmic microwave background (CMB) is nearly thermal and hence carries a local temperature that fluctuates on very small scales. This feature is captured in inflationary cosmology, where quantum fluctuations of some primordial field are believed to be the origin of the observed inhomogeneities [Wei08]. However, notions like temperature and "thermality" ultimately draw their justification from physical concepts which are based in Minkowski spacetime. Other examples for this include the homogeneous and isotropic models with matter content given by a perfect fluid or as dust, which - due to the equations of state involved - can be seen as thermodynamic in origin.1

\footnotetext{
${ }^{1}$ Another example for LTE in curved backgrounds is found in the use of Boltzmann's equation in big
} 
While there is the paradigm that certain thermal effects in the early universe allow for an "adiabatic" treatment, where one usually neglects curvature and uses Minkowskian concepts in the description of thermodynamics 2 , the question remains whether there is a notion of equilibrium which also captures possible effects due to curvature. It has to be local in nature and one is forced to ask if and in which way exactly notions from thermostatics of global equilibrium are recovered in curved spacetime ${ }^{3}$. We conclude that while in many regimes it is justified to use concepts which only make sense in QFT in Minkowski spacetime, it is important to formulate criteria for local equilibrium in the full quantum field theory on (cosmological) curved spacetimes, in order to check whether all features of the macroscopic physics are truly captured in these situations. For example, it could well be that LTE has something to say about the direction of the arrow of time or maybe forms of energy that are not accounted for in standard treatments [Buc03].

The definition of LTE states in [BOR02] and [BS07] makes use of point-like localized "thermal" observables, whose expectation values are compared with the corresponding expectation values when measured in a set of thermal reference states 4 . The state in question is considered to be in LTE if these values match, i.e. if it looks like a global equilibrium state (distinguished by the KMS condition) when tested with the thermal observables. The laws of thermostatics may be applied to the reference states and thus one may define spacetime dependent expectation values of interesting thermal functions in LTE states. Equations of state may also continue to hold, often taking the form of dynamical constraints on the spacetime dependence of the local thermal functions.

With the definition of LTE at hand, one is faced with an important conceptual problem: do LTE states exist in any physically meaningful QFT model? While there are interesting examples for LTE states in free field models, see e.g. [Buc03] for LTE in Minkowski spacetime and [Sch10] for LTE in a class of Robertson-Walker spacetimes 5 it is still important to prove the existence of LTE states in a model independent setting. We attack this problem in Chapter 2 of this thesis, with the following results. Under the assumption that there are no global linear equations of state, i.e. no linear relations between the macroscopic observables corresponding to the set of thermal observables, we

bang nucleosynthesis [KT90]. See [HL10] for a treatment of the Boltzmann equation in the context of QFT.

${ }^{2}$ The reason for this is that the interaction time scales are thought to be much larger than the corresponding curvature scales in the early universe. This includes discussions of Baryogenesis and Leptogenesis Wei08.

${ }^{3}$ For example, local equations of state ought to be influenced by curvature.

${ }^{4}$ The idea to attach thermal parameters to non-equilibrium states in curved spacetime by use of point-like thermal observables [BS07] requires use of locally covariant quantum fields, as developed in [HW01] and [BFV03], in order to compare measurements in different background spacetimes.

${ }^{5}$ Other examples have been discussed in [Bah06 Uec05 Hüb05 Sch05 Pet07. Sto09. 
establish existence of states which are in LTE in compact regions of Minkowski spacetime. Moreover, we find that the original definition of LTE in curved backgrounds given in [BS07] is too restrictive: one cannot use vector spaces of thermal observables to define LTE in curved spacetime. Instead, one has to use sets of thermal observables without any additional structure, meaning that linear combinations of thermal observables are not thermal observables again. As a consequence, we find interesting dynamical relations for the macroscopic observables in LTE states, which also take the background geometry into account. We also show existence of states which are in LTE at any given point in curved spacetime. In the absence of equations of state, they retain this property in a neighbourhood of that point.

A major assumption in our proof of existence of point-wise LTE states is unboundedness of linear combinations of the thermal observables (in the sense of quadratic forms). This is physically meaningful, since unboundedness of point-like quantum fields is expected due to the uncertainty relations. Using an argument by Fewster [Few05], we relate this to scaling limits of the corresponding quantum fields, which allows us to prove existence of point-wise LTE states for concrete sets of thermal observables in Chapter 3, where we deal with the free scalar field.

The thermal observables in this particular model are given by locally covariant Wick polynomials, which have been constructed in [HW01], up to certain universal renormalization constant: $\underbrace{6}$ due to the coupling to curvature. These ambiguities cannot be fixed without further input, which is reminiscent of the situation in particle physics, where quantities like mass and charge are renormalized and their values are fixed by experimental data. However, the situation is different here, because we lack simple observables in order to fix the values of the renormalization parameters that appear even in free theories in QFT in curved spacetime. However, as was observed in [BS07, the concept of LTE can be used to fix some of these numbers. Namely, as we have mentioned before, there exist interesting examples for LTE for the free scalar field: in particularly symmetric curved spacetimes, there are KMS states $\omega_{\beta}$ for certain classes of observers, and one is tempted to claim that at least some of these states should be in local equilibrium. Since the definition of local thermality depends on the renormalization constants (because the thermal observables do), this provides criteria to fix some of these numbers for all spacetimes.

We briefly illustrate this for the conformally coupled free scalar field $\left(-\square+\frac{1}{6} R\right) \phi=0$ and its locally covariant Wick square $: \phi^{2}$ :, which is a thermal observable corresponding to the local temperature $T(x)=\left(12 \omega\left(: \phi^{2}:(x)\right)\right)^{-\frac{1}{2}}$. The definition of $: \phi^{2}:$ is unique up to a constant $\alpha_{0} \in \mathbb{R}$ :

$$
: \phi^{2}: \rightarrow: \phi^{2}:+\alpha_{0} R
$$

\footnotetext{
"Universal" here means that these constants are the same for all spacetimes.
} 
where $R$ is the Ricci scalar. In (Anti) de Sitter spacetimes, one finds KMS states $\omega_{\beta}$ with respect to particular sets of observers in certain "wedges". Due to curvature, it is unclear whether the KMS parameter $\beta_{\mathrm{KMS}}=: 1 / T_{\mathrm{KMS}}$ can be interpreted as a temperature in the sense of the zeroth law. 7 Building on work by Buchholz and Schlemmer [BS07] and Stottmeister [Sto09], we make a proposal to fix $\alpha_{0}$ such that the conformal vacua in these spacetimes have a local temperature of zero. We are then able to relate $T_{\mathrm{KMS}}$ to the local temperature $T(x)$ (+ de Sitter, - Anti de Sitter):

$$
T_{\mathrm{KMS}}(x)=\sqrt{ \pm \frac{1}{(2 \pi \alpha)^{2}}+\frac{a^{2}(x)}{(2 \pi)^{2}}+T^{2}(x)} .
$$

The first term under the square root is a curvature term ( $\alpha$ denotes the radius of (Anti) de Sitter spacetime), the second includes the acceleration $a$ of the observer and the third term is the local temperature squared, as measured by devices that are gauged in global equilibrium states in Minkowski spacetime. This is a generalization of formulas found earlier in the literature [NPT96, DL97, Jac98].

Thus the constant $\alpha_{0}$ is fixed and hence a particular locally covariant Wick square has been chosen. It turns out, however, that for certain values of $\beta$ the intrinsic KMS $\omega_{\beta}$ states are nowhere in LTE, which cannot be avoided by any choice of $\alpha_{0}$. But this is expected, as these states, though passive with respect to some (in general non-geodesic) observers, are by no means guaranteed to have all the properties of equilibrium as required by the zeroth law. Formula 0.1 can be used to understand this situation: there are several contributions that have to contribute in a particular way in order to render a state KMS (and hence passive) with respect to the dynamics in question. These are displayed in 0.1 , and it is only the third contribution that is due to local thermality, while the geometry of the underlying spacetime and the motion of the observer account for the rest.

We also discuss sets of thermal observables of differentiated Wick powers such as the thermal energy tensor $\epsilon_{a b}$, which was introduced in [BOR02] already and adapted for use in curved spacetime in [SV08]. Using our definition of LTE without vector spaces of thermal observables and $\epsilon_{a b}$, we find interesting evolution equations for the local temperature in LTE states. We also discuss and adopt a new definition of the thermal energy tensor, a choice which we illustrate in examples from "intrinsic" KMS states in curved spacetime in Chapter 3 .

Finally, we also mention that a consistent definition of LTE can be seen as selection criterion to single out states of physical interest. For example, in QFT in curved spacetime, it is an important task to find states for the primordial quantum fields mentioned previously. Some interesting proposals exist which are of use in inflationary cosmology, e.g. low order adiabatic vacua used to predict the CMB-spectrum [Str06], which however

\footnotetext{
${ }^{7}$ I am grateful to Prof. D. Buchholz for pointing this out to me.
} 
lack physical motivation as states to which one may actually attach thermal attributes. Other proposals in case of free fields include states of low energy [Olb07] or minimal free energy [Küs08], which are better motivated but seem more difficult to handle in computations. Equilibrium-like states for free fields in cosmological spacetimes have also been constructed in [Hac10] and [DHP11], based on [DMP09]. However, it seems that without a good notion of LTE, thermal functions can be attached to these states only by some ad hoc procedure on a case-by-case basis. The notion of LTE discussed here may therefore help to clarify the local thermal properties of the states in these examples.

In this work, we use units where $\hbar=c=G=1$ and we usually measure temperature in units of energy, i.e. we put $k_{B}=1$. See [Wal84, Appendix F] for discussion and details on units. We use Einstein's summation convention and employ abstract indices $a, b, \ldots$ and indices $\mu, \nu, \ldots$ pertaining to a particular frame (coordinate or non-coordinate). We use the curvature conventions of Misner, Thorne \& Wheeler [MTW73] and Wald's book Wal84] (the conventions for curvature quantities are the same as in [HE73]). That is, our sign convention for the metric $g_{a b}$ is $(-,+,+,+)$ and in a local chart, the Riemann curvature tensor is given by

$$
R_{\mu \nu \rho}^{\sigma}=\Gamma_{\mu \rho, \nu}^{\sigma}-\Gamma_{\nu \rho, \mu}^{\sigma}+\Gamma_{\mu \rho}^{\alpha} \Gamma_{\alpha \nu}^{\sigma}-\Gamma_{\nu \rho}^{\alpha} \Gamma_{\alpha \mu}^{\sigma} .
$$

The Ricci tensor and Ricci scalar are defined by

$$
R_{a b}=R_{a c b}^{c} \text { and } R=g^{a b} R_{a b} .
$$




\section{Quantum Field Theory in Curved Spacetime}

In this chapter we recall preliminary material essential to this thesis. We provide basics on Quantum Field Theory in Curved Spacetime in its modern appearance, locally covariant Quantum Field Theory [BFV03], which is needed in order to discuss local thermal equilibrium in curved spacetime. Since we discuss local thermal equilibrium states for the Klein Gordon field in Chapter 3, we also provide some material on the free scalar field and the corresponding Wick powers.

The three classical textbooks on quantum field theory in curved spacetime are BD84, Ful89, Wal94, while a more recent treatment dealing also with locally covariant quantum field theory can be found in [BF09]. The material covered in this chapter is well known and details can be found in the literature as indicated.

\subsection{Locally Covariant Quantum Field Theory}

Quantum Field Theory (QFT) in Curved Spacetime describes quantum fields in the presence of gravitational fields in regimes where the quantum nature of gravity does not play an important rôle. While the back-reaction of the quantum fields on the metric is an important topic in this field of research $[$ it is usually neglected and we shall discuss neither the sources nor the dynamics of the gravitational field. Gravity is therefore described by a classical spacetime as in general relativity: it is a connected, oriented four-dimensional manifold $M$, equipped with a smooth Lorentzian metric $g$. Standard references include [HE73, MTW73] and [Wal84].

The initial successes that sparked interest in QFT in curved spacetime were the discovery of particle creation in expanding universes [Par69], the prediction of black hole radiation [Haw75] and - closely related - a finite temperature registered by observers that are uniformly accelerating through a vacuum state [Unr76]. A recent application was found in inflationary cosmology, where fluctuations of quantum fields are used to understand initial density fluctuations in the early universe, see [Wei08] for example.

\footnotetext{
${ }^{1}$ See for example [Hac10] and references therein.
} 
However, the study of QFT on manifolds is rewarding in itself; it has led to a deeper understanding for the structure of QFT and opened a clearer perspective on which parts of the theory are tied to specific spacetimes and their symmetries, and which are yet more fundamental. The existence of a vacuum state, a well defined particle picture and global equilibrium states in Minkowski spacetime are prime examples for structures that do not generalize straight-forwardly to curved spacetimes [Wa194]. It was learned that it is the algebra of observables that must be seen as more fundamental than these concepts - the construction of a theory has to proceed in a manner that is independent of particular states on this algebra ${ }^{2}$

More recently, it was even realized that there are tremendous conceptual advantages in constructing QFT simultaneously on a large class of spacetimes in accord with the principle of general covariance known from classical general relativity [HW01, BFV03]. This novel approach, known as locally covariant Quantum Field Theory (lcQFT), has led to new model independent results in QFT in curved spacetime such as the spin-statistics connection [Ver01], partial results on the Reeh-Schlieder property [San09], the analysis of superselection sectors [BR07, BR09] and new ideas on quantum energy inequalities [Few07, FP06]. Furthermore, the principles of locality and covariance have been essential in the perturbative construction of interacting QFT in curved spacetime [BF00], see also [HW01, HW02]. Moreover, new approaches to questions in cosmology have been found, see [DHMP10], [DFP08] and [DV10].

Since the definition of local thermal equilibrium in curved spacetime relies on locally covariant quantum fields [BS07, Sol10], we give an introduction to lcQFT here. Inspired in part by [Few11] and [HW10], we also give a (somewhat heuristic) motivation for its structure.

\subsubsection{Spacetime Geometry}

Before we begin our recapitulation of lcQFT, let us summarize some concepts from Lorentzian geometry ${ }^{3}$. Consider a Lorentzian manifold $(M, g)$. Choosing the signature of the metric $g$ as $(-,+,+,+)$, a vector $X \in T_{x} M$ is space-like if $g_{x}(X, X)>0$, time-like if $g_{x}(X, X)<0$ and light-like $g_{x}(X, X)=0$. The zero vector $X=0$ is defined to be space-like. A piecewise $C^{1}$-curve is space-like, time-like or light-like if its tangent vectors possess the corresponding property everywhere along the path. The class of causal curves consists of all piecewise $C^{1}$-curves which are time-like or light-like.

\footnotetext{
${ }^{2}$ This issue is relevant already when dealing with QFT in Minkowski spacetime (Algebraic QFT, see [Haa96]), but it becomes even more important in the presence of curvature.

${ }^{3}$ Apart from the references on general relativity mentioned before, we refer to [O'N83] and [BEE96] for detailed expositions.
} 
The set of time-like vectors at $x \in M$ consists of two connected components. We designate one of them as future and the other one as past. Doing so in a continuous fashion for all $x \in M$ yields a time orientation for the Lorentzian manifold $(M, g)$. Correspondingly, a time-like or light-like curve is called future (past) directed if its tangent vectors lie in the closure of the future (past) everywhere along the path. For later reference, we also define the future light cone $V_{x}^{+}$(past light cone $V_{x}^{-}$) at $x$, which consists of all future (past) directed light-like vectors in $T_{x} M$.

It is necessary to introduce some further causal structures. The causal future (past) $J_{+}^{M}(x)\left(J_{-}^{M}(x)\right)$ of a point $x \in M$ consists of $x$ itself and of all points which can be reached by a future (past) directed causal curve in $M$. We write

$$
J^{M}(x):=J_{+}^{M}(x) \cup J_{-}^{M}(x),
$$

which means that $J^{M}(x)$ comprises all points that can be reached by causal curves emerging from $x$. For a subset $\mathcal{O} \subset M$, we write

$$
J_{\ldots}^{M}(\mathcal{O}):=\cup_{x \in \mathcal{O}} J_{\ldots}^{M}(x) .
$$

Finally, we collect all information on the geometrical structure of a spacetime in the following notation.

Definition 1.1.1. The spacetime $\mathbf{M}$ consists of the Lorentzian manifold $(M, g)$ together with an orientation and a time orientation.

In lcQFT, the topological and geometrical data that constitute spacetime serve as background structure for the formulation of the theory. It is therefore natural to be interested in maps between spacetimes that preserve these structures in a suitable sense.

Definition 1.1.2. A map $\psi: \mathbf{M} \rightarrow \mathbf{N}$ is called hyperbolic embedding of $\mathbf{M}$ into $\mathbf{N}$ if it is an orientation and time orientation preserving isometry ${ }^{4}$ such that $\psi(\mathbf{M})$ is a causally convex subset of $\mathbf{N}$, i.e. every causal curve in $\mathbf{N}$ that begins and ends in $\psi(\mathbf{M})$ is contained wholly in $\psi(\mathbf{M})$.

If $\psi: \mathbf{M} \rightarrow \mathbf{N}$ is a hyperbolic embedding, one may view $\mathbf{N}$ as an enlargement of $\mathbf{M}$. In this regard it is worth noting that the natural embeddings of subspacetimes of a given spacetime are special cases of hyperbolic embeddings. Symmetries may also constitute hyperbolic embeddings, for example the proper orthochronous Poincaré transformations of Minkowski spacetime into itself. This particular symmetry is needed for the characterization of global equilibrium states in Chapter 2, because the action of the proper

\footnotetext{
${ }^{4}$ An isometry $\psi:(M, g) \rightarrow(N, h)$ is a diffeomorphism such that $\psi_{*} g=\left.h\right|_{\psi(M)}$ for the inverse pull-back $\psi_{*} g:=\left(\psi^{-1}\right)^{*} g$ of $g$.
} 
orthochronous Poincaré group symbolizes that there are inertial trajectories with respect to which a system can be in equilibrium.

With our geometrical notation in place, we are now in a position to start discussing the physical principles underlying lcQFT. Firstly, it seems natural to assume that physical experiments, if conducted in isolation from the rest of the world, range over a finite timespan and over finite spatial extent. Given a spacetime $\mathbf{M}$, a reasonable requirement is therefore that

$$
J_{+}^{M}(p) \cap J_{-}^{M}(q) \text { is compact for all } p, q \in M .
$$

Moreover, one would like to be able to distinguish a "before" and "after" for an experiment. A more formal statement is that $\mathbf{M}$ should not contain any closed causal curves. Technically, one should even impose the stronger condition that spacetime contains no "almost closed" causal curves. This is called strong causality, see [Wal84, Chapter 8.2] for a precise definition.

A further restriction on the geometry of $\mathbf{M}$ arises from the need to assign sensible dynamics to any physical system propagating on it, for example quantum fields. A subset of $M$ is called a Cauchy surface if each inextendible time-like curve intersects it at precisely one point. Cauchy surfaces are therefore reminiscent of surfaces of constant time in flat spacetime. If $\mathbf{M}$ contains a Cauchy surface $\Sigma$, any sensible notion of dynamics should thus allow prediction and retrodiction of the behaviour of the system on all of $\mathbf{M}$ by knowledge of suitable initial data on $\Sigma$.

Theorem 1.1.3. The following three conditions are equivalent:

(i) $\mathbf{M}$ is strongly causal and (1.1) holds.

(ii) There exists a Cauchy surface in $\mathbf{M}$.

(iii) $(M, g)$ is isometric to $\left(\mathbb{R} \times S,-f \mathrm{~d} t+h_{t}\right)$. Here, $f$ is a smooth positive function and $h_{t}$ is a Riemannian metric depending smoothly on $t$ and for each $t,\{t\} \times S$ is a smooth Cauchy surface for $M$.

Although known for a long time [HE73, Wal84, O'N83], a formal proof of this important result was completed only recently [BS05]. Any spacetime satisfying one (and hence all) of the three conditions is called globally hyperbolic. It can be seen from condition (iii) that on each globally hyperbolic spacetime $\mathbf{M}$ there exists a time function, i.e. a smooth function $t: M \rightarrow \mathbb{R}$ whose gradient is future-directed time-like at every $x \in M$ and all of whose level-sets 5 are Cauchy surfaces.

\footnotetext{
${ }^{5} \mathrm{By}$ this we mean the sets $t^{-1}(s) \subset M$ for $s$ in the range of $t$.
} 
In section 1.2 we review the initial value problem for normally hyperbolic differential operators like the Klein Gordon operator, which turns out to be well-posed on globally hyperbolic spacetimes [BGP07]. This is of importance for the construction of examples in Chapter 3.

One is thus led to consider the class of globally hyperbolic spacetimes as physically relevant background structure in the definition of lcQFT. Many prominent spacetimes like Minkowski spacetime $\mathbf{M}_{0}$, de Sitter spacetime and all of the Robertson-Walker spacetimes are in fact globally hyperbolic ${ }^{6}$

Definition 1.1.4. We define the category Loc, whose objects are globally hyperbolic spacetimes. Morphisms between objects $\mathbf{M}$ and $\mathbf{N}$ are hyperbolic embeddings of $\mathbf{M}$ into N.

\subsubsection{Observables and General Covariance}

In quantum theory, observables are represented by self-adjoint elements of a unital topological $*$-algebra $\mathcal{A}$, the algebra of observables. For mathematical convenience, $\mathcal{A}$ is usually taken as a $C^{*}$-algebra, but for us it is more suitable to consider algebras which are generated by polynomials of smeared quantum fields 7 , because the thermal observables used in the definition of local equilibrium are of this type. They typically cannot be represented by bounded operators.

Definition 1.1.5. We define the category Alg whose objects are unital topological *algebras $\mathcal{A}$. Morphisms are unit-preserving, continuous injective $*$-homomorphisms.

The existence of a morphism $\mathcal{A} \rightarrow \mathcal{A}^{\prime}$ means that $\mathcal{A}$ may be viewed as a subsystem of $\mathcal{A}^{\prime}$, i.e. morphisms represent embeddings of physical systems into larger ones.

The principle of "locality" expresses the idea that in the theoretical description of an experiment, the only "background structure" used in the construction of observables refers to a specific spacetime $\mathbf{M}$ and does not make use of data from the rest of any of the "larger" spacetimes $\mathbf{N}$ that possibly extend $\mathbf{M}$ (in the sense that there is a hyperbolic embedding $\psi: \mathbf{M} \rightarrow \mathbf{N}$ ). In a local theory, observables are therefore associated with spacetimes - and it is useful to keep in mind that in the previous example $\mathbf{M}$ can be an arbitrarily small neighbourhood of any point in $\mathbf{N}$.

For a given quantum system, locality implies that for any globally hyperbolic spacetime $\mathbf{M}$ there is an object in $\mathrm{Alg}$, denoted by $\mathcal{A}(\mathbf{M})$, that serves as the algebra of observables on $\mathbf{M}$. Let $\psi: \mathbf{M} \rightarrow \mathbf{N}$ be a hyperbolic embedding. Since $\mathbf{N}$ can be seen as an enlarged

\footnotetext{
${ }^{6}$ An important example that is not globally hyperbolic is given by Anti de Sitter spacetime.

${ }^{7}$ See [Sch90] for a mathematically precise treatment.
} 
version of $\mathbf{M}$ it is natural to assume that $\mathcal{A}(\psi(\mathbf{M})) \subset \mathcal{A}(\mathbf{N})$. Also, in a local theory no experiment taking place in $\mathbf{M}$ should depend on the spacetime structure outside of $\mathbf{M}$, so one expects a close relationship between $\mathcal{A}(\mathbf{M})$ and $\mathcal{A}(\psi(\mathbf{M}))$. Put differently, one expects the assignment $\mathbf{M} \mapsto \mathcal{A}(\mathbf{M})$ to behave "covariantly" under hyperbolic embeddings. This is close in spirit to the notion of "general covariance" in classical general relativity.

We can now construct a theory that is independent of any particular background spacetime, if we formulate it simultaneously on all globally hyperbolic spacetimes. Only then can we judge whether the theory is constructed locally and covariantly by testing how it behaves under a change of background structure, for example the metric.

This point of view is emphasized in the following definition [BFV03].

Definition 1.1.6. A locally covariant Quantum Field Theory is a covariant functor $\mathcal{A}$ from Loc to Alg.

A lcQFT $\mathcal{A}$ assigns to each spacetime $\mathbf{M}$ the algebra of observables $\mathcal{A}(\mathbf{M})$ for $\mathbf{M}$. If there is a hyperbolic embedding $\psi: \mathbf{M} \rightarrow \mathbf{N}$, functoriality means that the following diagram commutes:

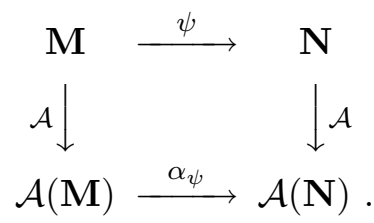

Here and henceforth, we write $\alpha_{\psi}$ instead of $\mathcal{A}(\psi)$. In case there are two hyperbolic embeddings $\mathbf{M} \stackrel{\psi}{\rightarrow} \mathbf{N} \stackrel{\psi^{\prime}}{\rightarrow} \mathbf{M}^{\prime}$, one has $\alpha_{\psi \circ \psi^{\prime}}=\alpha_{\psi} \circ \alpha_{\psi^{\prime}}$. Moreover, $\alpha_{\mathrm{id}_{\mathbf{M}}}=\mathrm{id}{ }_{\mathcal{A}(\mathbf{M})}$.

While the definition captures the aspects of locality and covariance from the previous discussion, it is necessary to supplement it with additional conditions on dynamics and causality.

Definition 1.1.7. Let $\mathcal{A}$ be a lcQFT. $\mathcal{A}$ obeys the time slice axiom if the following holds: for any morphism $\psi: \mathbf{M} \rightarrow \mathbf{N}$ such that $\psi(\mathbf{M})$ contains a Cauchy surface of $\mathbf{N}$, the map $\alpha_{\psi}: \mathcal{A}(\mathbf{M}) \rightarrow \mathcal{A}(\mathbf{N})$ is a $*$-isomorphism.

Moreover, let $\psi: \mathbf{M} \rightarrow \mathbf{N}$ and $\psi^{\prime}: \mathbf{M}^{\prime} \rightarrow \mathbf{N}$ be hyperbolic embeddings and assume that their images are space-like in $\mathbf{N}: J^{N}(\psi(\mathbf{M})) \cap \psi^{\prime}\left(\mathbf{M}^{\prime}\right)=\emptyset$. $\mathcal{A}$ is said to be causal if it holds that

$$
\left[\alpha_{\psi}(\mathcal{A}(\mathbf{M})), \alpha_{\psi^{\prime}}\left(\mathcal{A}\left(\mathbf{M}^{\prime}\right)\right)\right]=0.8
$$

The time slice axiom is the formal statement that there exists a dynamical law which allows prediction of the behaviour of the system just by knowledge of it in a neighbourhood of any Cauchy surface. Causality, on the other hand, ensures that observables which are space-like localized are commensurable in the sense of quantum theory. As is explained in

\footnotetext{
${ }^{8}$ Given two algebras $\mathcal{A}, \mathcal{A}^{\prime},\left[\mathcal{A}, \mathcal{A}^{\prime}\right]=0$ indicates $A A^{\prime}-A^{\prime} A=0 \forall A \in \mathcal{A}, A^{\prime} \in \mathcal{A}^{\prime}$.
} 
[BFV03, any lcQFT that obeys causality and fulfils the time-slice axiom may be viewed as a generalization of the Haag-Kastler approach to QFT in Minkowski spacetime [Haa96].

It is sometimes necessary to compare results of measurements with specific observables carried out at different spacetime locations 9 , possibly taking into account the motion of the apparatus with respect to local inertial frames. This is the main reason why in this thesis we work with locally covariant quantum field theory; in the definition of local thermal equilibrium in a curved spacetime $\mathbf{M}$, one compares expectation values of thermal observables in $\mathbf{M}$ with those of the "corresponding" observables in Minkowski spacetime $\mathbf{M}_{\mathbf{0}}$ [BS07]. For this purpose, a lcQFT $\mathcal{A}$ as such is not specific enough since it deals with algebras of observables 10 What is needed is a locally covariant definition of specific observables themselves, i.e. the notion of a locally covariant quantum field [HW01, BFV03].

These objects can be defined as natural transformations in the following way.

Definition 1.1.8. The objects of the category Top are topological spaces, while the morphisms are continuous maps. We define the functor

$$
\mathcal{D}: \text { Loc } \rightarrow \text { Top }
$$

that assigns to each object $\mathbf{M}$ in Loc the space of compactly supported smooth functions $\mathcal{D}(M)$, equipped with the usual topology. Hyperbolic embeddings $\psi: \mathbf{M} \rightarrow \mathbf{N}$ are mapped to their push forwards $\psi_{*}$, extended by 0 outside of $\psi(\mathbf{M})$.

Definition 1.1.9. Given a lcQFT $\mathcal{A}$, a locally covariant (scalar) quantum field $\phi$ is a natural transformation between the functors $\mathcal{D}$ and $\mathcal{A}$.

In plain terms, a locally covariant quantum field $\phi$ assigns to each globally hyperbolic spacetime $\mathbf{M}$ a quantum field $\phi_{\mathbf{M}}$. This means that on $\mathbf{M}, \phi_{\mathbf{M}}$ is a distribution taking values in the $*$-algebra $\mathcal{A}(\mathbf{M})$ :

$$
\mathcal{D}(M) \ni f \mapsto \phi_{\mathbf{M}}(f) \in \mathcal{A}(\mathbf{M}) .
$$

Locality and covariance are accounted for by the properties of a natural transformation. If $\psi: \mathbf{M} \rightarrow \mathbf{N}$ is a hyperbolic embedding, then it holds that

$$
\alpha_{\psi} \circ \phi_{\mathbf{M}}=\phi_{\mathbf{N}} \circ \psi_{*} .
$$

\footnotetext{
${ }^{9}$ This should be important in any statistical theory, as repetitions of measurements are done at different spacetime locations.

${ }^{10}$ It is an intriguing question whether a given lcQFT really describes "the same physics on all spacetimes" [FV11]. However, as we deal with specific fields instead of whole algebras here, this issue should not be relevant for the definition of local equilibrium.
} 
Thus $\phi$ is simply a family of quantum fields $\left\{\phi_{\mathrm{M}}\right\}$ indexed by all globally hyperbolic spacetimes M, i.e. a "master field" subject to the afore-mentioned transformation behaviour under hyperbolic embeddings. It is this viewpoint that allows comparison of "the same" measurements in different spacetimes, which is crucial in the definition of local equilibrium in curved spacetime.

More generally, we may consider a locally covariant $(r, s)$-tensor quantum field $\phi$. By this we mean that if $e$ is a local frame represented by a Lorentz tetrad $\left\{e_{\mu}\right\}_{\mu=0}^{3}$, then there exist locally covariant quantum fields

$$
\phi_{\mathbf{M}}{ }^{\mu_{1} \ldots \mu_{r}}{ }_{\mu_{1} \ldots \mu_{s}}: \mathcal{D}(M) \rightarrow \mathcal{A}(\mathbf{M})
$$

called the components of $\phi_{\mathrm{M}}$ in the frame $e$, which are subject to the usual transformation law of tensore 12

Finally, it should be noted that observable quantum fields are by definition real. In the scalar case this simply means that $\phi_{\mathbf{M}}(f)^{*}=\phi_{\mathbf{M}}(\bar{f})$, where ${ }^{*}$ is the $*$-operation in $\mathcal{A}(\mathbf{M})$ and $\bar{f}$ denotes the complex conjugate of $f$.

\subsubsection{States}

So far we have introduced observables and their locality properties. In order to describe measurements, however, one needs states - defined as expectation value functionals on the algebra of observables, see the discussion in [Ara00. Recall that, mathematically, a state of an algebra $\mathcal{A}$ with unit $\mathbb{1}$ is a continuous linear functional $\omega: \mathcal{A} \rightarrow \mathbb{C}$ which is positive, i.e. $\omega\left(A A^{*}\right) \geq 0$ for all $A \in \mathcal{A}$, and normalized, i.e. $\omega(\mathbb{1})=1$. If $\omega$ indeed models a physical state, then given an observable $A=A^{*}$, the number $\omega(A)$ is real and interpreted as the expectation value of a large number of measurements of $A$ on the system in the state $\omega$.

We denote the set of states of $\mathcal{A}$ by $\operatorname{Sts}(\mathcal{A})$. Note that $\operatorname{Sts}(\mathcal{A})$ is convex, i.e. if $\omega_{1}, \omega_{2} \in$ $\operatorname{Sts}(\mathcal{A})$ then

$$
\lambda \omega_{1}+(1-\lambda) \omega_{2} \in \operatorname{Sts}(\mathcal{A}) \text { for all } 0 \leq \lambda \leq 1
$$

We call a subset $\mathcal{S} \subset \operatorname{Sts}(\mathcal{A})$ closed under operations if it holds that if $\omega \in \mathcal{S}$ then

$$
\omega\left(A \cdot A^{*}\right) / \omega\left(A A^{*}\right) \in \mathcal{S} \text { for all } A \in \mathcal{A} \text { with } \omega\left(A A^{*}\right) \neq 0 .
$$

\footnotetext{
${ }^{11}$ This means that the tetrad is orthonormal with respect to $\operatorname{diag}(-1,+1,+1,+1)$ and $e_{0}$ is time-like future pointing.

${ }^{12}$ Other vector valued fields can also be accommodated in the locally covariant framework, as can locally covariant spinor fields, in which case one has to use the category of globally hyperbolic spacetimes with spin structure instead of Loc, see [San08] or [Hac10].
} 
We mention that each state gives rise to a Hilbert space representation of $\mathcal{A}$ via the well known GNS construction, see for example [Sch90], which allows recovery of the usual Hilbert space setting of quantum theory once a particular state has been chosen.

A class of states that are both mathematically and physically important are KMS states, defined here for later reference.

Definition 1.1.10. Let $t \mapsto \alpha_{t}$ be a one parameter group of automorphisms of $\mathcal{A}$. A state $\omega \in \operatorname{Sts}(\mathcal{A})$ is called $\left(\alpha_{t}, \beta\right)$-KMS state if for each pair of operators $A, B \in \mathcal{A}$ there is some function $F_{A, B}$ which is analytic in the strip $\{z \in \mathbb{C} \mid 0<\Im(z)<\beta\}$ and continuous at the boundaries such that

$$
F_{A, B}(t)=\omega_{\beta}\left(A \alpha_{t} B\right) \text { and } G_{A, B}(t):=F_{A, B}(t+i \beta)=\omega_{\beta}\left(\left(\alpha_{t} B\right) A\right) \text { for } t \in \mathbb{R} \text {. }
$$

KMS states are a natural generalization of the Gibbs ensembles used in quantum statistical mechanics [HHW67]. We further discuss their rôle as thermal reference states in Chapter 2 .

In view of a definition of states in the framework of lcQFT, we define the following category.

Definition 1.1.11. We define the category Sts whose objects are all convex subsets of $\operatorname{Sts}(\mathcal{A})$ which are closed under operations, for all objects $\mathcal{A}$ of Alg. Morphisms are given by affine maps, i.e. maps that preserve convex combinations.

While quantum fields can be locally covariant, it is important to realize that the idea of locally covariant states as a family of states $\left\{\omega_{\mathbf{M}}\right\}$, indexed by all globally hyperbolic spacetimes $\mathbf{M}$, is doomed to fail. States are non-local objects and as such do not transform in the desired way under hyperbolic embeddings. While individual states do not show the desired behaviour, there are sets of states which are in fact locally covariant [BFV03].

Definition 1.1.12. Given a lcQFT $\mathcal{A}$, let $\mathcal{S}$ be a contravariant functor from Loc to Sts such that $\mathcal{S}(\mathbf{M}) \subset \operatorname{Sts}(\mathcal{A}(\mathbf{M}))$ for all $\mathbf{M}$. $\mathcal{S}$ is called locally covariant state space for $\mathcal{A}$ if for each hyperbolic embedding $\psi: \mathbf{M} \rightarrow \mathbf{N}$ there holds

$$
\mathcal{S}(\psi)=\left.\alpha_{\psi}^{*}\right|_{\mathcal{S}(\mathbf{N})},
$$

where $\alpha_{\psi}^{*}$ denotes the dual map of $\alpha_{\psi}$. For each spacetime $\mathbf{M}$, we call $\mathcal{S}(\mathbf{M})$ the state space for $\mathbf{M}$.

A theory is thus specified by a pair of functors $(\mathcal{A}, \mathcal{S})$. However, one needs criteria for the physical interpretation of the states and local thermal equilibrium may prove to be important in this regard. 
Usually, quantum fields are rather singular objects and it is necessary to view them as operator valued distributions. However, we also need to discuss point-like localized quantum fields, as they are needed as thermal observables in the definition of local thermal equilibrium. As examples show (see the next section), with a suitable choice of locally covariant state space $\mathcal{S}$, one may be able to define point-like fields in the form sense as follows. Given a lcQFT $\mathcal{A}$ and a locally covariant quantum field $\phi$, let $\mathcal{S}$ be such that for each globally hyperbolic spacetime $\mathbf{M}$ and for each $\omega \in \mathcal{S}(\mathbf{M})$

$$
\omega\left(\phi_{\mathbf{M}}(f)\right)=\int_{M} \omega\left(\phi_{\mathbf{M}}(x)\right) f(x) \mathrm{d} \mu_{g}(x)
$$

for all $f \in \mathcal{D}(M)$ with some smooth function $x \mapsto \omega\left(\phi_{\mathbf{M}}(x)\right)$. For each $x \in M$ we may then define the point-like field $\phi_{\mathbf{M}}(x)$ as a linear form on the linear span of $\mathcal{S}(\mathbf{M})$. There is some literature regarding point-like fields in relation to algebras of observables in Minkowski spacetime, see [FH81] and [Bos00] in particular.

\subsection{The Free Scalar Field and Wick Polynomials}

A simple physically relevant example of a lcQFT arises by quantization of the free Klein Gordon field [BFV03, BGP07]. In the discussion of local equilibrium in the following chapters, we draw our examples from this theory.

\subsubsection{The Klein Gordon Equation and Quantization}

The Klein Gordon equation on a spacetime $\mathbf{M}$ can be derived from an action principle with action functional Wal84]

$$
S_{\mathrm{KG}}[\phi]:=\int_{M} \mathrm{~d} \mu_{g} L_{\mathrm{KG}}[\phi]
$$

with Lagrangian density

$$
L_{\mathrm{KG}}[\phi]:=\frac{1}{2} \nabla_{a} \phi \nabla^{a} \phi+\frac{1}{2}\left(\xi R+m^{2}\right) \phi^{2} .
$$

The resulting equation of motion is the Klein Gordon equation:

$$
P \phi:=\left(-\square+m^{2}+\xi R\right) \phi=0,
$$

with mass $m$ and curvature coupling $\xi \in \mathbb{R}$. Here $\square:=g^{a b} \nabla_{a} \nabla_{b}$ is the d'Alembert operator on $\mathbf{M}$. Regarding the choice of $\xi$, there are two special cases of interest: $\xi=0$, minimal coupling, and $\xi=\frac{1}{6}$, conformal coupling. The latter bears its name because the conformally coupled, massless $(m=0)$ Klein Gordon equation is invariant under conformal transformations of the metric, $g \mapsto \Omega^{2} g$ for any smooth function $\Omega: M \rightarrow \mathbb{R}$. We discuss this in more depth in chapter 3 . 
Before we proceed, let us introduce some standard notation regarding spaces of functions and distributions on $\mathbf{M} . \mathcal{E}(M)$ and $\mathcal{D}(M)$ denote the spaces of smooth functions $C^{\infty}(M)$ and smooth compactly supported functions $C_{0}^{\infty}(M)$ on $\mathbf{M}$ respectively, equipped with their usual locally convex topologies. $\mathcal{D}^{\prime}(M)$ denotes the space of distributions, i.e. the topological dual of $\mathcal{D}(M)$ consisting of continuous linear functionals $\mathcal{D}(M) \rightarrow \mathbb{C}$, whereas $\mathcal{E}^{\prime}(M)$ denotes the topological dual of $\mathcal{E}(M)$, i.e. the space of compactly supported distributions. Clearly $\mathcal{D}(M) \subset \mathcal{E}(M)$ and also $\mathcal{E}^{\prime}(M) \subset \mathcal{D}^{\prime}(M)$.

The theory of the Klein Gordon equation is well understood on globally hyperbolic spacetimes.

Theorem 1.2.1. We consider the Klein Gordon operator $P=-\square+m^{2}+\xi R$ on a globally hyperbolic spacetime $\mathbf{M}$.

1. Let $f \in \mathcal{D}(M), \Sigma$ be a smooth Cauchy surface of $M$ with future directed time-like unit normal vector field $\mathfrak{n}$ and $u_{0}, u_{1} \in \mathcal{D}(\Sigma)$. Then the Cauchy Problem

$$
P \phi=f, \quad \phi \uparrow_{\Sigma}=u_{0}, \quad \nabla_{\mathfrak{n}} \phi \uparrow_{\Sigma}=u_{1}
$$

has a unique solution $\phi \in \mathcal{E}(M)$ with $\operatorname{supp} \phi \subset J^{M}\left(\operatorname{supp} f \cup \operatorname{supp} u_{0} \cup \operatorname{supp} u_{1}\right)$.

2. There exist unique advanced $(+)$ and retarded (-) Green's operators $G_{ \pm}$for $P$, that are continuous linear maps $G_{ \pm}: \mathcal{D}(M) \rightarrow \mathcal{E}(M)$ with $(i) P \circ G_{ \pm}=\mathrm{id}_{\mathcal{D}(M)}$, (ii) $G_{ \pm} \circ P\left\lceil_{\mathcal{D}(M)}=\operatorname{id}_{\mathcal{D}(M)}\right.$ and $(i i i) \operatorname{supp}\left(G_{ \pm} \phi\right) \subset J_{ \pm}^{M}(\operatorname{supp} \phi)$ for all $\phi \in \mathcal{D}(M)$.

3. The maps $G_{+}$and $G_{-}$are formal adjoints of each other, i.e.

$$
\int_{M} f \cdot\left(G_{ \pm} g\right) \mathrm{d} \mu_{g}=\int_{M}\left(G_{\mp} f\right) \cdot g \mathrm{~d} \mu_{g} \forall f, g \in \mathcal{D}(M) .
$$

A detailed proof is given in [BGP07]. Results 1. and 2. are not confined to the Klein Gordon operator, but apply to any normally hyperbolic differential operator, i.e. a differential operator with metric principal part, written in local coordinates as

$$
P=-\square+A^{\mu} \partial_{\mu}+B
$$

with smooth functions $A^{\mu}$ and $B$. Result 3. of the theorem applies to any normally hyperbolic operator that is also formally selfadjoint.

Note that it is essential for the result that $\mathbf{M}$ is globally hyperbolic. In non-globally hyperbolic spacetimes, existence of advanced and retarded Green's operators is not guaranteed. If such maps do exist, they are in general not unique and one must supply additional boundary conditions. This accounts for the loss or influx of information during propagation of the system on the non-globally hyperbolic manifold. In chapter 3 we encounter Anti de Sitter spacetime as an example of this. 
Let us now define the causal Green's operator $G:=G_{-}-G_{+}$, which is a continuous map $\mathcal{D}(M) \rightarrow \mathcal{E}(M)$. Since $\mathcal{E}(M) \subset \mathcal{D}^{\prime}(M)$ and $G$ is continuous, the map

$$
\Delta(f, g):=\int_{M} f \cdot(G g) \mathrm{d} \mu_{g}, \quad f, g \in \mathcal{D}(M)
$$

defines a distribution $\Delta \in \mathcal{D}^{\prime}\left(M^{2}\right)$, which we call the causal propagator. By item 2 . of Theorem 1.2.1 one finds that $\Delta(f, g)$ vanishes whenever the supports of $f$ and $g$ are space-like separated. Result 3 . of the theorem shows that $\Delta$ is antisymmetric: $\Delta(f, g)=$ $-\Delta(g, f)$.

As is explained in many standard textbooks on QFT in Minkowski spacetime, see e.g. [IZ80], the idea of quantization is to replace the classical field observables with their quantum counterparts such that "equal time" canonical commutation relations (CCR) between the field and its canonical momentum are satisfied. More generally, in keeping with the relativistic symmetry, the commutator function should be given by the causal propagator. We have seen in theorem 1.2.1 that this object is well defined and unique on any globally hyperbolic spacetime. We make use of this fact in order to define an algebra of observables for the free scalar field on curved spacetime.

Definition 1.2.2. Let $\mathbf{M}$ be a globally hyperbolic spacetime. The Borchers-Uhlmann algebra for the free scalar field is defined as

$$
\mathcal{K}(\mathbf{M}):=\mathcal{B U}(\mathbf{M}) / \mathcal{J}
$$

where $\mathcal{B U}(\mathbf{M})$ is the algebraic direct sum ${ }^{13}$

$$
\mathcal{B U}(\mathbf{M}):=\bigoplus_{n=0}^{\infty} \mathcal{D}\left(M^{n}\right), \quad \mathcal{D}\left(M^{0}\right):=\mathbb{C},
$$

equipped with the following structures. Let $\underline{f}=\oplus_{l} f_{l}, \underline{g}=\oplus_{l} g_{l} \in \mathcal{B U}(\mathbf{M})$. We define:

1. a product $(\underline{f} \otimes \underline{g})_{n}\left(x_{1}, \ldots, x_{n}\right):=\sum_{l=0}^{n} f_{l}\left(x_{1}, \ldots, x_{l}\right) g_{n-l}\left(x_{l+1}, \ldots, x_{n}\right)$;

2. a $*$-operation $\left(\underline{f}^{*}\right)_{n}\left(x_{1}, \ldots, x_{n}\right):=\overline{f_{n}\left(x_{n}, \ldots, x_{1}\right)}$, extended antilinearly;

3. a topology such that a sequence $\left\{\underline{f}^{k}\right\}_{k}=\left\{\oplus_{l} f_{l}^{k}\right\}_{k}$ converges to $\underline{f}=\oplus_{l} f_{l}$ if $f_{l}^{k} \rightarrow f_{l}$ in the locally convex topology of $\mathcal{D}\left(M^{l}\right)$ for all $l$ and there exists an $N$ such that $f_{l}^{k}=0$ for all $l>N$ and all $k$.

Moreover, $\mathcal{J}$ is the closed $*$-ideal generated by elements of the form $-i \Delta(f, g) \oplus(f \otimes g-$ $g \otimes f)$ or $P f$. Finally, the $*$-algebra $\mathcal{A}(\mathbf{M})$ is equipped with product, *-operation and topology descending from $\mathcal{B U}(\mathbf{M})$.

\footnotetext{
${ }^{13}$ It consists of elements where only a finite number of terms in the sum are non-zero.
} 
The field itself is defined by

$$
\phi_{\mathbf{M}}(f):=[(0 \oplus f \oplus 0 \oplus \ldots)] \in \mathcal{K}(\mathbf{M})
$$

which implies in particular that $f \mapsto \phi_{\mathrm{M}}(f)$ is $\mathbb{C}$-linear. The map $\phi_{\mathrm{M}}$ is interpreted as a quantum field smeared with a test function $f$. Formally

$$
\phi_{\mathbf{M}}(f) “=" \int_{M} \phi_{\mathbf{M}}(x) f(x) \mathrm{d} \mu_{g}(x) .
$$

It should be noted that the equivalence classes corresponding to the quotient $\mathcal{B U}(\mathbf{M}) / \mathcal{J}$ are quite large. They contain functions with support in arbitrary small neighbourhoods of any Cauchy surface in $\mathbf{M}$, a consequence of the properties of $\Delta$ and the following well known result (see e.g. [San08, Lemma 3.1.16]).

Lemma 1.2.3. Let $\mathbf{M}$ be a globally hyperbolic spacetime and let $\mathcal{O}_{\Sigma}$ be a neighbourhood of a Cauchy surface $\Sigma$ in $\mathbf{M}$. Every $f \in \mathcal{D}(M)$ can be written as $f=g+$ Ph, where $g \in \mathcal{D}\left(\mathcal{O}_{\Sigma}\right)$ and $h \in \mathcal{D}(M)$.

This result is a strong statement about the dynamics of the free quantum field. For any $f \in \mathcal{D}(M)$ and any Cauchy surface $\Sigma$ in $M, \phi_{\mathbf{M}}(f)=\phi_{\mathbf{M}}(g)$ for some $g$ that is compactly supported in a neighbourhood of $\Sigma$. Roughly speaking, we can predict observables at any time if we know them in a small time interval. This leads to the conclusion that the lcQFT for the free scalar field obeys the time slice axiom (see Proposition 1.2.5).

Let us briefly show why the $*$-algebra $\mathcal{K}(\mathbf{M})$ is suitable for the description of the quantized real free scalar field ${ }^{14}$. Using the definition of $\phi_{\mathrm{M}}$, the $*$ - operation reads

$$
\left[\phi_{\mathbf{M}}\left(f_{1}\right) \ldots \phi_{\mathbf{M}}\left(f_{n}\right)\right]^{*}=\phi_{\mathbf{M}}\left(\overline{f_{n}}\right) \ldots \phi_{\mathbf{M}}\left(\overline{f_{1}}\right) \text {, }
$$

extended antilinearly to the whole algebra. The reality of the field is thus accounted for by the fact that $\phi_{\mathbf{M}}(f)^{*}=\phi_{\mathbf{M}}(f)$ for real-valued $f$. The set of observables consists of all field polynomials $\mathcal{P}$ with $\mathcal{P}=\mathcal{P}^{*}$.

Additionally, $\phi_{\mathrm{M}}$ obeys the Klein Gordon equation in the sense of distributions:

$$
\phi_{\mathbf{M}}(P f)=0
$$

for all $f \in \mathcal{D}(M)$. Lastly, the CCR read:

$$
\left[\phi_{\mathbf{M}}(f), \phi_{\mathbf{M}}(g)\right]:=\phi_{\mathbf{M}}(f) \phi_{\mathbf{M}}(g)-\phi_{\mathbf{M}}(g) \phi_{\mathbf{M}}(f)=i \Delta(f, g) \mathbb{1},
$$

where $\Delta$ is the causal propagator on $\mathbf{M}$. This is indeed a generalization of the usual equal time CCR, as can be seen from the following lemma [Dim80, Cor. 1.2] ${ }^{15}$

\footnotetext{
${ }^{14}$ There is also quantization of the real scalar field in terms of $C^{*}$-algebras, the so-called Weyl algebras, see [BGP07].

${ }^{15}$ See also the well-written exposition in [Hac10].
} 
Lemma 1.2.4. Let $\Sigma$ be a Cauchy surface for $\mathbf{M}$ with future directed time-like unit normal vector field $\mathfrak{n}$. For all $f \in \mathcal{D}(\Sigma)$ it holds that

$$
\nabla_{\mathfrak{n}} G f \uparrow_{\Sigma}=f \text { and } G f \uparrow_{\Sigma}=0 .
$$

Formally speaking, the lemma tells us that

$$
\nabla_{\mathfrak{n}} \Delta(x, y) \uparrow_{\Sigma}=\delta_{\Sigma}(x, y) \text { and } \Delta(x, y) \uparrow_{\Sigma}=0
$$

with $\delta_{\Sigma}$ denoting the delta distribution with respect to the metric-induced measure on $\Sigma$.

As we have mentioned, a Cauchy surface may be viewed as surface of constant time for some observer. Thus, if $x$ and $y$ are of equal time with regard to that observer, i.e. if $x, y \in \Sigma$, it holds that

$$
\begin{aligned}
{\left[\nabla_{\mathfrak{n}} \phi_{\mathbf{M}}(x), \phi_{\mathbf{M}}(y)\right] } & =i \nabla_{\mathfrak{n}} \Delta(x, y)=i \delta_{\Sigma}(x, y) \quad \text { and } \\
{\left[\phi_{\mathbf{M}}(x), \phi_{\mathbf{M}}(y)\right] } & =\Delta(x, y)=0
\end{aligned}
$$

on the level of formal distribution kernels. But these are just the usual "equal time" CCR for the "field" $\phi_{\mathrm{M}}(x)$ and its "canonical momentum" $\nabla_{\mathfrak{n}} \phi_{\mathrm{M}}(x)$.

For the construction of the lcQFT of the free scalar field, we note that if there is a hyperbolic embedding $\psi: \mathbf{M} \rightarrow \mathbf{N}$ between two globally hyperbolic spacetimes one may define an injective $*$-homomorphism $\mathcal{B U}(\mathbf{M}) \rightarrow \mathcal{B U}(\mathbf{N})$ determined by

$$
(0 \oplus f \oplus 0 \oplus \ldots) \mapsto\left(0 \oplus \psi_{*} f \oplus 0 \oplus \ldots\right),
$$

where $\psi_{*} f=f \circ \psi^{-1}$, extended by 0 outside of $\psi(\mathbf{M})$. One can show that this map descends to an injective $*$-homomorphism $\alpha_{\psi}: \mathcal{A}(\mathbf{M}) \rightarrow \mathcal{A}(\mathbf{N})$ [San08, Prop. 3.1.10].

Proposition 1.2.5. We define the functor $\mathcal{K}$ from Loc into Alg that assigns to each object $\mathbf{M}$ in Loc the Borchers-Uhlmann algebra of the free scalar field $\mathcal{K}(\mathbf{M})$ and to each morphism $\psi: \mathbf{M} \rightarrow \mathbf{N}$ the injective $*$-homomorphism $\alpha_{\psi}: \mathcal{K}(\mathbf{M}) \rightarrow \mathcal{K}(\mathbf{N})$.

It follows that $\mathcal{K}$ is a lcQFT which is causal and obeys the time slice axiom. Moreover, $\mathbf{M} \mapsto \phi_{\mathbf{M}}$ is a locally covariant quantum field.

The result is well known for the Weyl algebraic approach [BFV03, BGP07] and details on the unbounded case are presented in [San08].

\subsubsection{Hadamard States and the Extended Algebra of Observables}

The lcQFT of the free scalar field given by the functor $\mathcal{K}$ is not sufficient for many purposes. In perturbative $Q F T$, where a non-linear interacting theory is approximated 
by a formal expansion around the corresponding linear theory, the quantities of physical interest are Wick polynomials and their time ordered products, i.e. fields that are generated by products of the basic field and their derivatives, evaluated at the same spacetime point. These objects are formally infinite and therefore are not elements of $\mathcal{K}(\mathbf{M})$. They must be defined via a suitable generalization of normal ordering, known from textbook QFT in Minkowski spacetime [IZ80].

While perturbative QFT is not discussed in this thesis, we note that even for the description of the free theory, the functor $\mathcal{K}$ is not satisfactory. One of the most important physical observables, the energy momentum tensor, is a Wick polynomial and is therefore not included in $\mathcal{K}(\mathbf{M})$. What is most important for us is that thermal observables are among the Wick polynomials (e.g. the Wick square, which turns out to be a good thermometer). We thus review the construction of an enlargement of the algebra $\mathcal{K}(\mathbf{M})$ which also includes these fields. It turns out that a suitable restriction on the "small distance" (i.e. "high energy") behaviour of the states of $\mathcal{K}(\mathbf{M})$ is needed, i.e. a replacement of the spectrum condition known from QFT in Minkowski spacetime.

A state $\omega$ of $\mathcal{K}(\mathbf{M})$ is determined by its $n$-point functions

$$
\omega^{(n)}\left(f_{1}, \ldots, f_{n}\right):=\omega\left(\phi_{\mathbf{M}}\left(f_{1}\right) \ldots \phi_{\mathbf{M}}\left(f_{n}\right)\right) .
$$

By the continuity of $\omega$ and the Schwartz kernel theorem, the $\omega_{n}$ are distributions in $\mathcal{D}^{\prime}\left(M^{n}\right)$.

Definition 1.2.6. A state $\omega$ on $\mathcal{A}(\mathbf{M})$ is called even if it is invariant under the transformation $\phi_{\mathbf{M}}(f) \mapsto-\phi_{\mathbf{M}}(f) \sqrt[16]{16}$ A state $\omega$ is called quasi-free, if the $\omega^{(n)}$ for odd $n>1$ vanish and, moreover,

$$
\omega^{(2 n)}\left(f_{1}, \ldots, f_{2 n}\right)=\sum_{\pi \in \Pi_{n}} \omega^{(2)}\left(f_{\pi(1)}, f_{\pi(2)}\right) \ldots \omega^{(2)}\left(f_{\pi(2 n-1)}, f_{\pi(2 n)}\right)
$$

where $\Pi_{n}$ is the set of permutations of $\{1, \ldots, 2 n\}$ with $\pi(1)<\pi(3)<\cdots<\pi(2 n-1)$ and $\pi(2 i-1)<\pi(2 i), i=1, \ldots, n$.

Quasi-free states are closely related to a Fock space picture, see e.g. [BR96 ${ }^{17}$.

One defines normally ordered products with respect to any quasi-free state $\omega$ on $\mathcal{K}(\mathbf{M})$

\footnotetext{
${ }^{16}$ Clearly, the $n$-point functions of an even state vanish for odd $n$.

${ }^{17}$ Quasi-free states are also called Gaussian states, because they satisfy $\omega\left(e^{i \phi_{\mathbf{M}}(f)}\right)=\exp \left(-\frac{1}{2} \omega^{(2)}(f, f)\right)$ in the sense of formal power series.
} 
recursively via the relations

$$
\begin{aligned}
: \phi_{\mathrm{M}}: \stackrel{\text { def. }}{=} \phi_{\mathbf{M}} & \\
: \phi_{\mathbf{M}}\left(x_{1}\right) \ldots \phi_{\mathbf{M}}\left(x_{n+1}\right): \stackrel{\text { def. }}{=}: & \phi_{\mathbf{M}}\left(x_{1}\right) \ldots \phi_{\mathbf{M}}\left(x_{n}\right): \phi_{\mathbf{M}}\left(x_{n+1}\right) \\
& \quad-\sum_{i=1}^{n}: \phi_{\mathbf{M}}\left(x_{1}\right) \ldots \check{\phi_{M}}\left(x_{i}\right) \ldots \phi_{\mathbf{M}}\left(x_{n}\right): \omega^{(2)}\left(x_{i}, x_{n+1}\right),
\end{aligned}
$$

where indicates that the corresponding factor is omitted. Note that the normally ordered products

$$
W_{n}\left(x_{1}, \ldots, x_{n}\right) \stackrel{\text { def. }}{=}: \phi_{\mathbf{M}}\left(x_{1}\right) \ldots \phi_{\mathbf{M}}\left(x_{n}\right):
$$

are symmetric in all of their arguments and, when smeared with test functions, are elements of $\mathcal{K}(\mathbf{M})$. The sought for enlargement of $\mathcal{K}(\mathbf{M})$ is generated by $W_{n}$ smeared not only with test functions, but also with certain compactly supported distributions. This is because Wick products are defined by restriction of the $W_{n}$ to the diagonal $x_{1}=\cdots=x_{n}$, which can be achieved by smearing $W_{n}$ with the distribution $f \delta_{n}$. Here $f \in \mathcal{D}(M)$ and $\delta_{n} \in \mathcal{D}^{\prime}\left(M^{n}\right)$ is the "diagonal distribution"

$$
\int h\left(x_{1}, \ldots, x_{n}\right) \delta_{n}\left(x_{1}, \ldots, x_{n}\right) \mathrm{d} \mu_{g}\left(x_{1}\right) \ldots \mathrm{d} \mu_{g}\left(x_{1}\right)=\int h(x, \ldots, x) \mathrm{d} \mu_{g}(x) .
$$

Thus the definition of Wick powers involves taking the pointwise product of distributions, which is in general ill-defined.

In Minkowski spacetime $\mathbf{M}_{\mathbf{0}}$, normal ordering can be done with the help of the distinguished vacuum state $\omega_{\infty}$ on $\mathcal{A}\left(\mathbf{M}_{\mathbf{0}}\right)$. The state $\omega_{\infty}$ is quasi-free and in the massless case, which we consider here for simplicity, the corresponding two-point function is given by

$$
\omega_{\infty}^{(2)}(x, y)=\lim _{\varepsilon \rightarrow 0} \frac{1}{4 \pi^{2}} \frac{1}{(x-y)^{2}+i \varepsilon\left(x_{0}-y_{0}\right)+\varepsilon^{2}}
$$

in global inertial coordinates, where $(x-y)^{2}$ is the Minkowski inner product derived from the Minkowski metric. Note that 1.15 is to be understood in the sense of the the usual $\varepsilon$-prescription, i.e. the limit must be taken after smearing with test functions.

We see that the two-point function is smooth for space-like and time-like related $x$ and $y$, while it singular for $(x-y)^{2}=0$. Loosely speaking, this indicates that the product of fields $\phi_{\mathbf{M}_{\mathbf{0}}}(x) \phi_{\mathbf{M}_{\mathbf{0}}}(y)$ is singular at $(x-y)^{2}=0$ and the square of the field must be defined using normal ordering, i.e. by "subtraction of the singularity" $\sqrt{18}$, The result is that Wick polynomials can be evaluated in any state $\omega$ with the property that $\omega^{(2)}-\omega_{\infty}^{(2)}$ is sufficiently regular.

\footnotetext{
${ }^{18}$ This is equivalent to the more commonly known reordering of creation and annihilation operators in momentum space in the usual Fock space picture from which normal ordering derives its name.
} 
However, since the Wick polynomials are elements of an algebra, we must also worry about their products. Wick's Theorem [IZ80] states that, schematically,

$$
\begin{aligned}
W_{n} \cdot W_{m} & =W_{n+m}+\sum_{1 \text { contraction }} \omega^{(2)} W_{n+m-2} \\
& +\sum_{2 \text { contractions }} \omega^{(2)} \omega^{(2)} W_{n+m-4}+\ldots,
\end{aligned}
$$

where a contraction means the suppression of two arguments in the subsequent normal products. We see that by 1.16 the product of two Wick powers contains powers of $\omega_{\infty}^{(2)}(x, y)$.

In Minkowski spacetime, one may use techniques from Fourier analysis in order to show objects like $\omega_{\infty}^{(2)}(x, y)^{n}$ are well defined distributions. One finds that the Fourier transform of $w(x-y):=\omega_{\infty}^{(2)}(x, y)$ has support in the positive light cone. This is a special instance of the spectrum condition [Haa96] and it is this fact that makes it possible to define $\omega_{\infty}^{(2)}(x, y)^{n}$ by $n$-fold convolution of the Fourier transforms of $w$ [RS75].

A general curved spacetime $\mathbf{M}$ possesses no translation symmetry and hence one cannot make use of global Fourier techniques. In general, the notion of a vacuum state does not exist 19 and there is no global analogue of the spectrum condition. It was found, however, that the spectrum condition finds a local analogue in curved spacetime using techniques from micro-local analysis.

We provide some basics on micro-local analysis and additionally refer the reader to the standard monograph [Hör90] or to [RS75, Str09, BF00] for introductory accounts. It is a standard result that a distribution $u \in \mathcal{E}^{\prime}\left(\mathbb{R}^{n}\right)$ is smooth if and only if its Fourier transform decays rapidly, i.e. for any $n \in \mathbb{N}$ there exist constants $C_{n}$ such that

$$
|\widehat{u}(k)| \leq C_{n}(1+|k|)^{-n}
$$

for all $k \in \mathbb{R}^{n} \backslash\{0\}$. Here $|k|$ denotes the Euclidean norm of $k$.

If $u$ is not smooth the Fourier transform may still decrease rapidly in certain "regular directions". The set of these directions is an open cone in $\mathbb{R}^{n} \backslash\{0\}$ and moreover, it is stable if we multiply $u$ by some $f \in \mathcal{D}\left(\mathbb{R}^{n}\right)$. Since for any $f \in \mathcal{D}\left(\mathbb{R}^{n}\right)$ and $u \in \mathcal{D}^{\prime}\left(\mathbb{R}^{n}\right)$ the product $f \cdot u$ is a distribution with compact support, this suggests a strategy on how to define regular directions in the general case when $u \in \mathcal{D}^{\prime}\left(\mathbb{R}^{n}\right)$.

Definition 1.2.7. A regular direction for a distribution $u \in \mathcal{D}^{\prime}\left(\mathbb{R}^{n}\right)$ is a point $(x, k) \in$ $\mathbb{R}^{n} \times\left(\mathbb{R}^{n} \backslash 0\right)$ for which there exist an $f \in \mathcal{D}\left(\mathbb{R}^{n}\right)$ with $f(x) \neq 0$, a coni ${ }^{20}$ open neighbourhood $V \subset \mathbb{R}^{n} \backslash 0$ of $k$ and constants $C_{n}$ for all $n \in \mathbb{N}$ such that

$$
|\widehat{(f \cdot u)}(k)| \leq C_{n}(1+|k|)^{-n}
$$

\footnotetext{
${ }^{19}$ See [Wal94] for further elaboration on this point.

${ }^{20}$ Recall that an open subset $V \subset \mathbb{R}^{n}$ is called conic if $q \in V$ entails that $\lambda q \in V$ for all $\lambda>0$.
} 
for all $k \in V$. The wave front set $\mathrm{WF}(u)$ of a distribution $u \in \mathcal{D}^{\prime}\left(\mathbb{R}^{n}\right)$ is defined as $\mathrm{WF}(u):=\left\{(x, k) \in \mathbb{R}^{n} \times\left(\mathbb{R}^{n} \backslash 0\right) \mid(x, k)\right.$ is not a regular direction for $\left.u\right\}$.

The wave front set not only encodes the singular support of a distribution but also the directions in Fourier space in which the distribution fails to be rapidly decreasing. If $u \in \mathcal{D}^{\prime}\left(\mathbb{R}^{n}\right)$ is smooth, $\operatorname{WF}(u)=\emptyset$. Moreover, $\operatorname{WF}(D u) \subset \mathrm{WF}(u)$ for any partial differential operator $D$ and $\operatorname{WF}(f u) \subset \operatorname{WF}(u)$ for smooth $f$.

The notion of a wave front set of a distribution can be lifted to any smooth manifold.

Lemma 1.2.8. The wave front set transforms covariantly under diffeomorphisms as a subset of $T^{*} \mathbb{R}^{n}$. One can therefore extend its definition to distributions on general manifolds $M$ by patching together the wave front sets in different coordinate patches of $M$. For $u \in \mathcal{D}^{\prime}(M)$ one finds $\mathrm{WF}(u) \subset T^{*} M \backslash\{0\}$, where 0 denotes the zero section of $T^{*} M$.

The mathematically precise condition concerning when the pointwise product of distributions exists makes use of wave front sets.

Theorem 1.2.9. Let $u, v \in \mathcal{D}^{\prime}(M)$ and define

$$
\mathrm{WF}(u) \oplus \mathrm{WF}(v):=\{(x, k+l) \mid(x, k) \in \mathrm{WF}(u), \quad(x, l) \in \mathrm{WF}(v)\} .
$$

If $\mathrm{WF}(u) \oplus \mathrm{WF}(u)$ does not contain the zero section of $T^{*} M$, then one can define the pointwise product $u \cdot v \in \mathcal{D}^{\prime}(M)$ with $\mathrm{WF}(u \cdot v) \subset \mathrm{WF}(u) \cup \mathrm{WF}(v) \cup \mathrm{WF}(u) \oplus \mathrm{WF}(v)$. If $u$ and $v$ are smooth, $u \cdot v$ reduces to the usual pointwise product between smooth functions.

The wave front set, cf. Definition 1.2.7, of $\omega_{\infty}^{(2)}$ is given by [RS75, Theorem IX.48]

$$
\begin{aligned}
\mathrm{WF}\left(\omega_{\infty}^{(2)}\right)=\{(x, y, k,-k) & \left.\in T^{*} M_{0}^{2} \mid x \neq y,(x-y)^{2}=0, k \|(x-y), k_{0}>0\right\} \\
& \cup\left\{(x, x, k,-k) \in T^{*} M_{0}^{2} \mid k^{2}=0, k_{0}>0\right\} .
\end{aligned}
$$

Theorem 1.2 .9 confirms that e.g. $\omega_{\infty}^{(2)}(x, y)^{2}$ is well defined, since $\mathrm{WF}\left(\omega_{\infty}^{(2)}\right) \oplus \mathrm{WF}\left(\omega_{\infty}^{(2)}\right)$ does not contain the zero section. It is shown in [BFK96] that higher powers of $\omega_{\infty}^{(2)}$ are also well defined.

This result suggests that one should seek a generalization of (1.17) as a selection criterion for states that allow a definition of Wick products in curved spacetime ${ }^{21}$

Definition 1.2.10. Let $\omega$ be a state on $\mathcal{K}(\mathbf{M})$. We say that $\omega$ is a Hadamard state if its two-point function $\omega^{(2)}$ fulfils the Hadamard condition, that is

$$
\mathrm{WF}\left(\omega^{(2)}\right)=\left\{\left(x, x^{\prime}, k,-k^{\prime}\right) \in T^{*}(M)^{2} \mid(x, k) \sim\left(x^{\prime}, k^{\prime}\right), k \text { is future directed }\right\} .
$$

Here, $(x, k) \sim\left(x^{\prime}, k^{\prime}\right)$ means that there is a light-like geodesic connecting $x$ to $x^{\prime}$ to which $k$ and $k^{\prime}$ are cotangent at $x$ and $x^{\prime}$ respectively, with $k^{\prime}$ being the parallel transport of $k$. When $x=x^{\prime}$, we require $k=k^{\prime}$.

\footnotetext{
${ }^{21}$ This method of introducing Hadamard states is inspired by [Hac10].
} 
Hadamard states exist on any globally hyperbolic spacetime [FNW81]. Moreover, the set of Hadamard states on a given spacetime is closed under operations [San08, Prop. 3.1.9.], so we can define a locally covariant state space, i.e. a contravariant functor $\mathcal{S}$ that maps globally hyperbolic spacetimes to the set of Hadamard states $\mathcal{S}(\mathbf{M})$ on $\mathcal{K}(\mathbf{M})$.

It is important to note is that the difference between the two-point functions of two Hadamard states is smooth. This is why the expectation values of Wick powers (wrt. some quasi-free Hadamard state $\omega$ ) exist in any Hadamard state $\omega^{\prime}$, i.e. we may restrict $\omega^{\prime}\left(W_{n}\left(x_{1}, \ldots, x_{n}\right)\right)$ to the total diagonal $x_{1}=\cdots=x_{n}$. Thus it follows that Wick powers and their derivatives may be defined as point-like fields, i.e. as linear forms on the linear span of $\mathcal{S}(\mathbf{M})$. This is crucial for the present work: as was mentioned before, the thermal observables are Wick polynomials and it is therefore important that we are able to measure them at points.

In order to discuss the algebraic structure of the normally ordered products, let us define the following subset of $\mathcal{E}^{\prime}\left(M^{n}\right), n \in \mathbb{N}$ :

$$
\begin{aligned}
\mathcal{E}^{\prime}{ }_{n}(\mathbf{M}):=\left\{t \in \mathcal{E}^{\prime}\left(M^{n}\right) \mid\right. & t \text { is symmetric, } \\
& \left.\operatorname{WF}(t) \subset\left(T^{*} M\right)^{n} \backslash\left(\bigcup_{x \in M}\left(V_{x}^{+}\right) \cup \bigcup_{x \in M}\left(V_{x}^{-}\right)\right)\right\} .
\end{aligned}
$$

By definition, the sought for extension of $\mathcal{K}(\mathbf{M})$ consists of the unit $\mathbb{1} \in \mathcal{K}(\mathbf{M})$ and normally ordered products smeared with elements from $\mathcal{E}^{\prime}\left(M^{n}\right)$. It is the condition on the wave front set of these distributions that guarantees that the smeared objects are well defined [BF00]. By Wick's Theorem, the normally ordered products are equipped with the associative product

$$
W_{n}(f) \cdot W_{m}(g)=\sum_{k=0}^{\min (m, n)} W_{m+n-2 k}\left(f \otimes_{k} g\right),
$$

with the symmetrized, $k$-times contracted tensor product

$$
\begin{array}{r}
\left(f \otimes_{k} g\right)\left(x_{1}, \ldots, x_{n+m-2 k}\right) \stackrel{\text { def. }}{=} \mathbf{S} \frac{n ! m !}{(n-k) !(m-k) ! k !} \int_{M^{2 k}} \mathrm{~d} \mu_{g}\left(y_{1}\right) \ldots \mathrm{d} \mu_{g}\left(y_{2 k}\right) \\
\omega^{(2)}\left(y_{1}, y_{2}\right) \ldots \omega^{(2)}\left(y_{2 k-1}, y_{2 k}\right) f\left(x_{1}, \ldots, x_{n-k}, y_{1}, y_{3}, \ldots, y_{2 k-1}\right) \times \\
g\left(x_{n-k+1}, \ldots, x_{n+m-2 k}, y_{2}, y_{4}, \ldots, y_{2 k}\right),
\end{array}
$$

where $\mathbf{S}$ denotes symmetrization in $x_{1}, \ldots, x_{n}$. Once again, the form of the wave front set of the smearing distributions entails that this product is well-defined [HW01]. A *-operation is given by $W(f)^{*}=W(\bar{f})$, extended anti-linearly.

The previously sketched construction seems to depend on the Hadamard state $\omega$ used in the definition of the normally ordered products $W_{n}$. However, as has been shown in [HW01, Lemma 2.1], different choices for $\omega$ lead to $*$-isomorphic algebras. Using different 
states just amounts to using a different set of generators for the same abstract algebra, which we denote by $\mathcal{W}(\mathbf{M})$ henceforth.

Definition 1.2.11. The *-algebra $\mathcal{W}(\mathbf{M})$ constructed above will be referred to as the extended algebra of observables for the free scalar field on the globally hyperbolic spacetime M.

The extended algebra of observables $\mathcal{W}(\mathbf{M})$ can be equipped with a notion of convergence of sequences, based on so-called Hörmander pseudo-topologies [BF00, HW01, HR02], which has the property that $\mathcal{K}(\mathbf{M})$ is dense in $\mathcal{W}(\mathbf{M})$.

The assignment $\mathbf{M} \mapsto \mathcal{W}(\mathbf{M})$ is a lcQFT [HW01, Lemma 3.1], which moreover fulfils the time slice axiom [CF09]. It follows from results in [HR02] and San10 that continuous 22 states on $\mathcal{W}(\mathbf{M})$ are in one-to-one correspondence to Hadamard states on $\mathcal{K}(\mathbf{M})$ that are extended to $\mathcal{W}(\mathbf{M})$. We continue to call these states Hadamard states. Thus it is justified to regard the set of Hadamard states $\mathcal{S}(\mathbf{M})$ as the suitable space of states on the extended algebra $\mathcal{W}(\mathbf{M})$ and we will look for LTE states in $\mathcal{S}(\mathbf{M})$ in Chapter 3

A slightly different formulation of the above constructions, allowing a unified description of the algebraic structure of the classical and the quantum field theory, goes under the name deformation quantization. An early reference for this approach is [DF01].

\subsubsection{Locally Covariant Wick Polynomials}

In this section we describe how Wick polynomials can be constructed in a locally covariant manner. Again, this is of importance, because in the definition of LTE one needs locally covariant thermal observables. Candidates for Wick monomials of all orders can be found in the enlarged algebra $\mathcal{W}(\mathbf{M})$, because the diagonal distribution $\delta_{n}$ is an element of $\mathcal{E}^{\prime}{ }_{n}(\mathbf{M})$ for all $n$. Given a quasi-free Hadamard state $\omega$, one may define

$$
: \phi^{n}:{ }_{\omega}(f):=W_{n}\left(f \delta_{n}\right)
$$

for $f \in \mathcal{D}(\mathbf{M})$, cf. equations 1.12, 1.13. In this section, we drop reference to the spacetime $\mathbf{M}$ from the notation for the fields, i.e. we write $\phi$ instead of $\phi_{\mathbf{M}}$.

One may also define derivatives of Wick monomials [Mor03], for example:

$$
: \phi \nabla_{\mu} \phi: \omega_{\omega}(f):=W_{2}(t) \quad \text { with } \quad t\left(x_{1}, x_{2}\right):=-\nabla_{\mu}^{\left(x_{2}\right)}\left(f\left(x_{1}\right) \delta_{2}\left(x_{1}, x_{2}\right)\right) .
$$

Recall that $\mathrm{WF}(\partial u) \subset \mathrm{WF}(u)$ and $\mathrm{WF}(f u) \subset \mathrm{WF}(u)$ when $f$ is smooth, so that the distribution $t$ is a well defined element of $\mathcal{E}^{\prime}{ }_{2}(\mathbf{M})$. More generally, we consider the formal adjoint [BGP07] of the differential operator we seek to apply to the normally ordered field

\footnotetext{
${ }^{22}$ Here, the previously mentioned notion of convergence is to be understood.
} 
and apply it to the smearing distribution "(test function) $\cdot \delta_{n}$ “. Fields such as $: \nabla_{\mu} \phi \nabla_{\nu} \phi$ : can be defined with the help of parallel transport in a natural way, see [Hac10], for example. It is worth noting that the Leibniz rule applies, e.g. $\nabla_{\mu}: \phi^{2}: \omega=2: \phi \nabla_{\mu} \phi:_{\omega}$.

The previous definitions have the major defect that they do not give rise to locally covariant quantum fields. The construction of fields like $: \phi^{n}:_{\omega}$ depends on the Hadamard state $\omega$, which is an inherently non-local object, see [HW01, BFV03] for details. In order to remedy this shortcoming, we first introduce a more concrete characterization of Hadamard states, which is also more suited for performing calculations with them.

For this purpose, we define the half squared geodesic distance, also sometimes called Synge's world function. On a geodesically convex set $\mathcal{O}$ in $M$, by definition for each $x \in \mathcal{O}$ there is a set $\mathcal{O}_{x} \subset T_{x} M$ such that the exponential map $\exp _{x}: \mathcal{O}_{x} \rightarrow \mathcal{O}$ is a well-defined diffeomorphism. Every globally hyperbolic spacetime can be covered with such sets ['N83]. On such $\mathcal{O}$, we define the half squared geodesic distance by

$$
\sigma(x, y):=\frac{1}{2} g_{x}\left(\exp _{x}^{-1}(y), \exp _{x}^{-1}(y)\right)
$$

for $x, y \in \mathcal{O}$. Note that $\sigma: \mathcal{O} \times \mathcal{O} \rightarrow \mathbb{R}$ is smooth and symmetric.

Given a time function $t$ on $\mathbf{M}$, we define

$$
\sigma_{\varepsilon}(x, y):=\sigma(x, y)+2 i \varepsilon(t(x)-t(y))+\varepsilon^{2} .
$$

Definition 1.2.12. A bi-distribution $b \in \mathcal{D}^{\prime}(M \times M)$ is said to be of local Hadamard form if, for every $x_{0} \in M$, there exists a geodesically convex neighbourhood $\mathcal{O}$ of $x_{0}$ such that on $\mathcal{O} \times \mathcal{O}$

$$
b(x, y)=\lim _{\varepsilon \searrow 0} \frac{1}{8 \pi^{2}}\left(h_{\varepsilon}(x, y)+w(x, y)\right) .
$$

Here $h_{\varepsilon}$ defines the Hadamard parametrix $h:=\lim _{\varepsilon \backslash 0} h_{\varepsilon}$ at some arbitrary scale $\lambda \in \mathbb{R}$,

$$
h_{\varepsilon}(x, y):=\frac{u(x, y)}{\sigma_{\varepsilon}(x, y)}+v(x, y) \log \left(\frac{\sigma_{\varepsilon}(x, y)}{\lambda^{2}}\right)
$$

where the Hadamard coefficients $u, v$ and $w$ are smooth, real-valued bi-scalars.

This definition is a special case of the definition of the global Hadamard form given in [KW91, see also [SV01] for a detailed exposition. The global condition ensures that there are no singularities in addition to the light-like ones that are found explicitly in the local form and that the definition is independent of the choice of time function $t$.

If the bi-distribution in question is the two-point function $\omega^{(2)}$ of a state $\omega$, it satisfies positivity, $\omega^{(2)}(\bar{f}, f) \geq 0$ for all $f \in \mathcal{D}(M)$, has the causal propagator $\Delta$ as its antisymmetric part and is a bi-solution to the Klein Gordon equation. Remarkably, as has been 
shown in $\left[\mathrm{Rad} 96 \mathrm{a}\right.$, these conditions entail that if $\omega^{(2)}$ has local Hadamard form, it also has global Hadamard form.

An even more remarkable result, also due to Radzikowski [Rad96b], is the following ${ }^{23}$

Theorem 1.2.13. Let $\omega$ be a state on $\mathcal{A}(\mathbf{M})$. Then $\omega$ is Hadamard in the sense of definition 1.2 .10 if and only if the two-point function $\omega^{(2)}$ is of global Hadamard form.

By the above discussion, we can replace the term "global" with "local" in this theorem. Therefore, the rather abstract characterization of Hadamard states in terms of micro-local analysis is indeed equivalent to the more concrete realization that was used much earlier in the renormalization of the energy momentum tensor, see [Wal94] and references therein.

The fact that $\omega^{(2)}$ is also bi-solution of the Klein Gordon equation allows one to draw strong conclusions about the Hadamard coefficients. Usually, $v$ is expressed in terms of a series expansion in $\sigma$ :

$$
v=\sum_{n=0}^{\infty} v_{n} \sigma^{n},
$$

where the $v_{n}$ are smooth bi-scalar coefficients. This expansion is convergent on analytic spacetimes, but not necessarily so on smooth spacetimes. Therefore, the series is usually truncated at some order $n$ and in turn one requires $w$ in 1.26 only to be of regularity $C^{n}$.

The property $P_{x} \omega^{(2)}=0$ together with the fact that we have $\omega^{(2)}=h+w$ for some smooth $w$ entails that

$$
P_{x} h=-P_{x} w,
$$

and hence $P_{x} h$ must be smooth. This condition is satisfied if terms proportional to $\sigma^{-1}$ and $\ln \sigma$ in $P_{x} h$ vanish and this, in turn, is achieved if Hadamard's recursion relations are satisfied.

Lemma 1.2.14. The functions $u$ in 1.27 and $v_{n}$ in 1.28, $n \geq 0$, obey the Hadamard recursion relations:

$$
\begin{gathered}
2 \nabla_{a} u \nabla^{a} \sigma+(\square \sigma-4) u=0 \\
P_{x} v_{n}+(n+1)\left(2 \nabla_{a} v_{n+1} \nabla^{a} \sigma+(\square \sigma+2 n) v_{n+1}\right)=0 \quad \forall n \geq 0 .
\end{gathered}
$$

Here, all derivatives are taken with respect to the first entry.

Together with the initial condition $u(x, x)=1$, this leads to the result that $u$ is given by the van Vleck-Morette determinant and one also finds further conditions on the coincidence limits $v_{n}(x, x)$ which serve as initial conditions for the equations involving the $v_{n}$. The

\footnotetext{
${ }^{23}$ See also [SV01.
} 
resulting initial value problems have uniquely determined smooth solutions, see [DB60, Ful89, PPV11] or [BGP07].

It follows that $u$ is solely determined by the local geometry of the spacetime, while $v$ also depends on the parameters $\xi$ and $m^{2}$ present in the Klein Gordon operator $P$. Therefore, the singular part of the two-point function $\omega^{(2)}$ of a Hadamard state $\omega$ is, in fact, state independent and the state dependent information of the two-point function is contained in the smooth function $w$, which is also symmetric by the CCR.

Following [HW01], we can now define Wick powers in a locally covariant manner ${ }^{24}$ In the definition of the normal ordered products $W_{n}(1.12$, we replace the two-point function $\omega^{(2)}$ with the Hadamard parametrix $h$. In this way, we have traded the non-local object $\omega^{(2)}$ for a purely local quantity that has the same wave front set so that smearing with elements of $\mathcal{E}^{\prime}{ }_{n}(\mathbf{M})$ is still well defined. Note, however, that $h$ is defined in a geodesic normal neighbourhood $\mathcal{O}$, so strictly speaking we have defined : $\phi^{n}: h(f)$ for $f \in \mathcal{D}(\mathcal{O})$ only. However, a global definition can be given using a partition of unity argument [Mor03]. When considering Wick powers as point-like fields, this does not matter, because only coincidence limits are relevant for the definition. The series expansion of $v$ in $\sigma$ may not converge on smooth but non-analytic spacetimes. This is not an issue, however, in the coincidence limit: one truncates the series at order $n$, where $n$ is the order of the highest derivative that appears in the Wick monomial in question.

We have identified elements of the extended algebra of observables $\mathcal{W}(\mathbf{M})$ as candidates for locally covariant Wick monomials. According to [HW01], the latter are expected to fulfil certain physically motivated requirements. Firstly, there should be a notion of continuity (analyticity) of the fields under smooth (analytic) variations of the metric and the coupling parameters $\xi, m^{2}$. Secondly, the fields should exhibit a certain behaviour under rescaling of the metric and $\xi, m^{2}$. Hollands and Wald have shown that Wick monomials satisfying the aforementioned requirements are unique up to certain local curvature terms [HW01, Theorem 5.1]:

$$
: \phi^{n}:(x)=: \phi^{n}: h(x)+\sum_{k=0}^{n-2}\left(\begin{array}{l}
n \\
k
\end{array}\right) C_{n-k}(x): \phi^{k}: h(x),
$$

where $: \phi^{i}:_{h}(x)$ are the Wick powers defined previously with the help of the Hadamard parametrix $h$. The functions $C_{i}(x)$ are polynomials with real coefficients in the metric, curvature and $m^{2}$, which scale as $C_{i}(x) \mapsto \mu^{i} C_{i}(x)$ under rescalings $g \mapsto \mu^{-2} g, m^{2} \mapsto \mu^{2} m^{2}$ and $\xi \mapsto \xi$. The real coefficients mentioned here thus constitute the renormalization freedom for locally covariant Wick polynomials. Similar conclusions can be drawn for

\footnotetext{
${ }^{24}$ See [HW02] for local and covariant time ordered products.
} 
Wick powers including derivatives. The most important observable of this type is the energy momentum tensor $T_{a b}$, which we discuss in the final part of this chapter in order to prepare the ground for Chapters 2 and 3

The classical field $T_{a b}^{\mathrm{cl}}$ is computed by varying the action 1.2 with respect to the metric [Wal84]:

$$
T_{a b}^{\mathrm{cl}}:=\frac{2}{\sqrt{|\operatorname{det}(g)|}} \frac{\delta S_{\mathrm{KG}}}{\delta g^{a b}} .
$$

The result can be written as

$$
\begin{aligned}
T_{a b}^{\mathrm{cl}}=\frac{1}{2} \nabla_{a} \nabla_{b} \phi^{2} & -\phi \nabla_{a} \nabla_{b} \phi-\frac{1}{2} g_{a b}\left(\frac{1}{2} \square \phi^{2}+m^{2} \phi^{2}-\phi \nabla^{c} \nabla_{c} \phi\right) \\
& +\xi\left(G_{a b} \phi^{2}-\nabla_{a} \nabla_{b} \phi^{2}+g_{a b} \square \phi^{2}\right) .
\end{aligned}
$$

Using the equations of motion (1.4), one can easily show that $\nabla^{a} T_{a b}^{\mathrm{cl}}=0$ and $g^{a b} T_{a b}^{\mathrm{cl}}=$ $-\left(3\left(\frac{1}{6}-\xi\right) \square+m^{2}\right) \phi^{2}$. In stationary spacetimes, the first equation amounts to conservation of the energy measured by observers following time-like Killing trajectories. The second identity implies tracelessness of $T_{a b}^{\mathrm{cl}}$ for the massless, conformally coupled scalar field.

We obtain a candidate for a locally covariant quantum field $T_{a b}$ that corresponds to the stress energy tensor by replacing the monomials $\phi^{2}$ and $\phi \nabla_{a} \nabla_{b} \phi$ in 1.33 with the Wick monomials : $\phi^{2}$ : and $: \phi \nabla_{a} \nabla_{b} \phi$ : respectively. The results by Hollands and Wald [HW01] imply that this prescription is unique up to the following renormalization freedom:

$$
: \phi^{2}:=: \phi^{2}: h+C \text { and }: \phi \nabla_{a} \nabla_{b} \phi:=: \phi \nabla_{a} \nabla_{b} \phi: h+C_{a b}
$$

where $C=\alpha_{0} R+\alpha_{1} m^{2}$ with real constants $\alpha_{0}, \alpha_{1}$ and $C_{a b}$ is any symmetric tensor constructed out of the metric, (derivatives of) curvature and $m^{2}$ with dimension length ${ }^{-4}$.

Making use of the Leibniz rule and recalling $P:=-\square+m^{2}+\xi R$, one calculates

$$
\nabla^{a} T_{a b}=-:\left(\nabla_{b} \phi\right) P \phi:
$$

and

$$
g^{a b} T_{a b}=-\left(3\left(\frac{1}{6}-\xi\right) \square+m^{2}\right): \phi^{2}:-: \phi P \phi: .
$$

The right hand side of 1.35 and the second term of the right hand side of 1.36 vanish for the classical field due to the equations of motion. If we had used Wick powers constructed with respect to some quasi-free Hadamard state $\omega$, then Wick products containing a factor $P \phi$ would vanish. This is easily verified using the fact that $\omega^{(2)}$ is a bi-solution for $P$. However, the Hadamard parametrix $h$ in general does not have this property and one computes Mor03.

$$
: \phi P \phi: h=\frac{3}{4 \pi^{2}}\left[v_{1}\right] \mathbb{1} \text { and }:\left(\nabla_{b} \phi\right) P \phi: h=\frac{1}{4 \pi^{2}} \nabla_{b}\left[v_{1}\right] \mathbb{1},
$$


where $\left[v_{1}\right](x):=v_{1}(x, x)$ denotes the coincidence limit of the Hadamard coefficient $v_{1}$. $\left[v_{1}\right]$ is a smooth function consisting of local curvature terms, which can be found for example in [DF06]. Equation 1.37 shows that $: \phi P \phi: h$ and $:\left(\nabla_{b} \phi\right) P \phi: h$ are state-independent quantities, but in contrast to classical field theory these expressions do not vanish.

The fact that a locally covariant Wick monomial which contains a factor $P \phi$ does not vanish is the origin of so-called anomalies, which are well known from earlier attempts to define a quantum energy momentum tensor ("Wald's axioms", see [Wa194] and references therein). In any prescription, the covariant conservation law $\nabla^{a} T_{a b}=0$ is regarded as being crucial, especially when trying to give sense to the semi-classical Einstein equations [Hac10]. We are left with the renormalization freedom 1.34 to deal with the anomalous terms in 1.35 and 1.36.

$C$ and $C_{a b}$ must therefore be chosen such that $:\left(\nabla_{b} \phi\right) P \phi:=0$. The resulting constraint is [HW05]

$$
\begin{aligned}
C_{a b}-\frac{1}{2} g_{a b} C_{c}^{c} \stackrel{!}{=} & \frac{1}{4 \pi^{2}}\left[v_{1}\right] g_{a b}+\left(\frac{1}{2}-\xi\right) \nabla_{a} \nabla_{b} C \\
& -\left(\frac{1}{4}-\xi\right) g_{a b} \square C+\left(\xi G_{a b}-\frac{1}{2} m^{2} g_{a b}\right) C-T_{a b}^{R F} .
\end{aligned}
$$

Here, $T_{a b}^{R F}$ is the most general divergence-free symmetric tensor of the correct dimension that depends solely on the curvature, the metric and $m^{2}$ :

$$
T_{a b}^{R F}=\beta_{1} I_{a b}+\beta_{2} J_{a b}+\beta_{3} m^{2} G_{a b}+\beta_{4} m^{4} g_{a b}
$$

with real constants $\beta_{i}$ and the two independent conserved local curvature terms

$$
\begin{aligned}
I_{a b} & :=g_{a b}\left(\frac{1}{2} R^{2}+2 \square R\right)-2 \nabla_{a} \nabla_{b} R-2 R R_{a b} \quad \text { and } \\
J_{a b} & :=\frac{1}{2} g_{a b}\left(R_{c d} R^{c d}+\square R\right)-\nabla_{a} \nabla_{b} R+\square R_{a b}-2 R_{c d} R_{a b}^{c}{ }_{b} .
\end{aligned}
$$

If we define

$$
\begin{aligned}
& T_{a b}^{h}:=\frac{1}{2} \nabla_{a} \nabla_{b}: \phi^{2}:_{h}-: \phi \nabla_{a} \nabla_{b} \phi:{ }_{h}-\frac{1}{2} g_{a b}\left(\frac{1}{2} \square: \phi^{2}:_{h}+m^{2}: \phi^{2}:_{h}-: \phi \nabla^{c} \nabla_{c} \phi: h\right) \\
&+\xi\left(G_{a b}: \phi^{2}:_{h}-\nabla_{a} \nabla_{b}: \phi^{2}:_{h}+g_{a b} \square: \phi^{2}:_{h}\right),
\end{aligned}
$$

then the quantum energy momentum tensor, renormalized according to the constraint (1.38), is computed to be

$$
T_{a b}:=\left\{T_{a b}^{h}+\frac{1}{4 \pi^{2}}\left[v_{1}\right] g_{a b} \mathbb{1}\right\}+T_{a b}^{R F} \mathbb{1} .
$$

By construction $\nabla^{a} T_{a b}=0$, and the trace reads

$$
g^{a b} T_{a b}=-\left(3\left(\frac{1}{6}-\xi\right) \square+m^{2}\right): \phi^{2}:_{h}-\frac{1}{4 \pi^{2}}\left[v_{1}\right] \mathbb{1}+g^{a b} T_{a b}^{R F} \mathbb{1} .
$$

\footnotetext{
${ }^{25}$ The term in parentheses in equation [1.42] is Moretti's prescription [Mor03] for the definition of the quantum energy momentum tensor.
} 
Note that $T_{a b}^{R F}$ cannot be chosen such that the state independent terms in 1.43 cancel each other. This means in particular that in the conformally coupled, massless case $T_{a b}$ is not traceless. This is often referred to as the conformal anomaly. It can be shown that anomalies cannot be avoided when dealing with locally covariant Wick polynomials [HW05]. Nevertheless, this discussion shows that physical principles like the conservation of the energy momentum tensor can be used to reduce the possible renormalization freedom appearing in the definition of locally covariant Wick products. As we discuss in chapter 3 , there may be a possibility to fix the remaining free constants with the help of thermal data, as proposed in [BS07]. Another possibility to restrict the range of the constants with the help of the semi-classical Einstein equation and data from cosmology is described in [DHMP10].

The constructions which we have reviewed in this section show that the physically interesting observables for the quantized free scalar field can be constructed on globally hyperbolic spacetimes, in accord with the principles of locality and covariance. The thermal observables used in Chapter 3 are among them. 


\section{Local Thermal Equilibrium}

This chapter deals with the definition of local thermal equilibrium (LTE) states in a modelindependent setting. In Section 2.1 we review the definition of LTE states given by Buchholz, Ojima and Roos [BOR02], and we discuss its generalization to curved spacetime [BS07] in Section 2.2. In this chapter we also present our own results on the structure of the set of thermal observables and on the existence of LTE states. Most of these results have been published in [Sol10].

\subsection{Local Thermal Equilibrium States in Minkowski Spacetime}

\subsubsection{Basic Definitions}

In quantum field theory global equilibrium states are to be described by use of the KMS condition [Haa96], cf. Definition 1.1.10. Global equilibrium states have interesting properties, among them the fact that only a few global parameters play a rôle in the description of their macroscopic behaviour, for example temperature. Apart from being subject to the laws of thermostatics, these macroscopic observables also usually obey certain equations of state.

Many macroscopic systems of interest, however, are at best locally close to an equilibrium situation (hydrodynamic flows, steady states, perturbations of equilibrium), and one cannot expect to be able to assign global thermal parameters to them. Instead one should be able to attach local thermal parameters to such states, for example a temperature parameter that is spacetime dependent. At the same time, equations of state should become "local" in the sense that they are possibly replaced by evolution equations for the local macroscopic observables. The definition given in [BOR02] captures these aspects of local equilibrium.

Before we give a definition of LTE based on BOR02, we need to recall the characterization of global thermal equilibrium for QFT in Minkowski spacetime. In view of a generalization of LTE to curved spacetime, we assume that we are dealing with a locally covariant quantum field theory $\mathcal{A}$ with locally covariant state space $\mathcal{S}$. We use abbrevia- 
tions like $\mathcal{A}_{0}:=\mathcal{A}\left(\mathbf{M}_{\mathbf{0}}\right)$ and $\mathcal{S}_{0}:=\mathcal{S}\left(\mathbf{M}_{\mathbf{0}}\right)$ for objects in Minkowski spacetime $\mathbf{M}_{\mathbf{0}}$.

$\mathbf{M}_{\mathbf{0}}$ is maximally symmetric and hence there is a ten-dimensional symmetry group acting on $\mathbf{M}_{\mathbf{0}}$ by hyperbolic embeddings. It is the proper orthochronous Poincaré group $\mathcal{P}_{+}^{\uparrow}:=\mathbb{R}^{4} \rtimes \mathcal{L}_{+}^{\uparrow}$, i.e. the semi-direct product of translations and the proper orthochronous Lorentz group $\mathcal{L}_{+}^{\uparrow}$. Since $\mathcal{A}$ is a lcQFT, there is an automorphic action $\mathcal{P}_{+}^{\uparrow}$ on $\mathcal{A}_{0}$ :

$$
\mathcal{P}_{+}^{\uparrow} \ni(a, \Lambda) \mapsto \alpha_{a, \Lambda} \in \operatorname{Aut}\left(\mathcal{A}_{0}\right)
$$

In the case of a pure translation, we write $a \mapsto \alpha_{a}$ instead of $\alpha_{a, 1}$.

The dynamics of an inertial observer is given by time translations, i.e. time-like isometries $\iota_{t}(x)=x+t e$ for some $e \in \mathbb{R}^{4}$ with $e^{2}=-1$ and $e^{0}>0$. The corresponding one-parameter group of automorphisms acting on $\mathcal{A}_{0}$ is $t \mapsto \alpha_{t e}$. If a state $\omega_{\beta} \in \mathcal{S}_{0}$ is a $\left(\alpha_{t e}, \beta\right)$-KMS state $2^{2}$ for some time-like unit vector $e^{2}=-1, e^{0}>0$, it is viewed as a global equilibrium state at temperature $T=\frac{1}{\beta}$ by any inertial observer moving along a trajectory $\gamma$ with $\gamma^{\prime}=e$.

The justification for this is found in the fact that KMS states are generalizations of finite volume Gibbs ensembles to the infinite medium [HHW67]. There are also several other results that relate natural characterizations of global equilibrium to KMS states, either by their behaviour under small perturbations of the dynamics [HB74], their characterization via passivity, related to the second law of thermodynamics [PW78], or through the principle of detailed balance and the zeroth law [KFGV77]. See [BR96] for more information on KMS states.

As in [BOR02], we encode the information on the rest frame $e$ and on the inverse temperature $\beta \in \mathbb{R}^{+}$into a single time-like vector $\beta \cdot e$, again denoted by $\beta$, the temperature vector. The set of KMS states at any given temperature vector is known to form a simplex, which implies that the elements of these sets can be distinguished with the help of central observables ("classical" observables) such as chemical potentials or some mean energy density.

Let us denote by $V^{+}$the set of vectors $v$ with $v^{2}<0$ and $v^{0}>0$. In the following, we assume for simplicity that to each $\beta \in V^{+}$there is exactly one KMS state. Physically, this means that we consider systems with a single phase only. One of the consequences of this assumption is a simple behaviour of the KMS states under Poincaré transformations. Namely, given a KMS state $\omega_{\beta}$ and a transformation $(\Lambda, a) \in \mathcal{P}_{+}^{\uparrow}$, the transformed state is by definition $\omega_{\beta} \circ \alpha_{\Lambda, a}^{-1}$. From the KMS condition it follows that $\omega_{\beta} \circ \alpha_{\Lambda, a}^{-1}$ is a KMS state with temperature vector $\Lambda \beta$. Therefore, by the uniqueness assumption

$$
\omega_{\beta} \circ \alpha_{\Lambda, a}^{-1}=\omega_{\Lambda \beta}
$$

\footnotetext{
${ }^{1}$ See for example [Haa96 for details.

${ }^{2}$ Recall Definition 1.1 .10
} 
This means that the $\omega_{\beta}$ are invariant under translations. In their rest systems, they are also isotropic.

We also assume that the functions $\beta \mapsto \omega_{\beta}(A)$ are continuous for all $A \in \mathcal{A}_{0}$. Thus, mixtures of KMS states may be formed by means of integration with suitable measures $\rho$ on $V^{+}$. The set $\mathcal{C} \subset \mathcal{S}_{0}$ of reference states consists of states

$$
\omega_{\rho}:=\int \mathrm{d} \rho(\beta) \omega_{\beta}
$$

Reference states are generally not KMS states, but they retain the property of passivity [PW78] and a suitable generalization of thermostatics applies to them [BOR02].

Since by assumption for each $\beta \in V^{+}$there exists a unique KMS state $\omega_{\beta}$, every intensive thermodynamic quantity $F$ attached to these states (thermal energy density, entropy density etc.) can be expressed as a function of $\beta$ alone. These functions $\beta \mapsto F(\beta)$ are called thermal functions. As explained before, they characterize the macroscopic properties of the KMS states $\omega_{\beta}$ completely. Thus we may write $F(\beta)=\omega_{\beta}(F)$ and interpret $F$ as a macroscopic (central) observable. Evaluated in a reference state $\omega_{\rho} \in \mathcal{C}$, one obtains the mean value of $F$ with regard to the measure $\rho$, cf. equation 2.2 :

$$
\omega_{\rho}(F)=\int d \rho(\beta) F(\beta) .
$$

Hence, thermodynamic quantities may also be attached to the reference states.

The basic idea for the definition of LTE states is that an ensemble which is in LTE at a given point $x \in \mathbf{M}_{\mathbf{0}}$ should not be distinguishable from some (mixture of) global thermal equilibrium states with regard to measurements performed with "thermal observables" localized at $x$. These thermal observables are quantum fields which can be point-like localized. Let us therefore assume that $\mathcal{A}_{0}$ is generated by a set of locally covariant quantum fields, denoted by $\phi_{\mathrm{M}_{\mathbf{0}}}=: \phi_{0}$ etc. . We also assume that the state space $\mathcal{S}_{0}$ is chosen such that the $\phi_{0}(x)$ can be defined as linear forms on the linear span of $\mathcal{S}_{0}$ for all $x$, as described at the end of section 1.1.3.

Given a quantum field $\phi_{0}(x)$ we define the corresponding functions

$$
\beta \mapsto \Phi(\beta):=\omega_{\beta}\left(\phi_{0}(x)\right) .
$$

Note that by translation invariance of the KMS states, the right hand side does not depend on $x \in \mathbf{M}_{\mathbf{0}}$. Moreover, it follows from our assumptions that the functions $\Phi$ are continuous. To see this, take any test function $f$ with $\int f(x) \mathrm{d}^{4} x=1$. By assumption, $\omega_{\beta}\left(\phi_{0}(f)\right)$ is continuous in $\beta$ and by translation invariance of the KMS states, one has $\omega_{\beta}\left(\phi_{0}(f)\right)=\omega_{\beta}\left(\phi_{0}(x)\right)=\Phi(\beta)$, hence $\beta \mapsto \Phi(\beta)$ is continuous.

As is discussed in [BOR02], not every quantum field in $\mathcal{A}_{0}$ is a good candidate for being a thermal observable, as not all fields are sensitive to the "thermal properties" of 
the system. It seems reasonable to choose the set of thermal observables $\mathbf{T}_{\mathbf{0}}$ such that one may reproduce (or at least approximate) all relevant thermal functions $F$ by use of equation 2.3, schematically:

$$
F(\beta)=" \lim _{i} \Phi^{(i)}(\beta) "
$$

for some sequence $\phi_{0}^{(i)} \in \mathbf{T}_{\mathbf{0}}$. This provides a consistent link between the microscopic and the macroscopic properties of the reference states. A simple example would be a "local thermometer", i.e. a scalar quantum field $\phi_{0}(x)$ such that the corresponding $\Phi(\beta)$ is a simple function of $\beta$ that may be inverted. Thus the field $\phi_{0}(x)$ allows one to distinguish KMS states at different temperatures $T=\frac{1}{\beta}$, but in order to distinguish the rest frames $e \in V^{+}$, one also needs tensor fields.

Let us assume that a set $\mathbf{T}_{\mathbf{0}}$ of thermal observables has been chosen and that the functions corresponding to these fields by $(2.3)$ are physically relevant thermal functions.

Definition 2.1.1. A state $\omega \in \mathcal{S}$ is called $\mathbf{T}_{\mathbf{0}}(x)$-thermal if there exists a normalized measure $\rho_{x}$, supported in $V^{+}$, such that

$$
\omega(\phi(x))=\omega_{\rho_{x}}(\phi(x))
$$

for all $\phi(x) \in \mathbf{T}_{\mathbf{0}}(x)$.

Note that the $x$-dependence of the right-hand side of 2.4 lies entirely in the measure $\rho_{x}$, since the reference states in $\mathcal{C}$ are translationally invariant by our uniqueness assumption.

The reason why we regard $\mathbf{T}_{\mathbf{0}}(x)$-thermal states as LTE states is that we may attach thermal functions to them, depending on the size of $\mathbf{T}_{\mathbf{0}}$. To see this, note that we can lift any $\mathbf{T}_{\mathbf{0}}(x)$-thermal state $\omega$ to a linear functional on the space of thermal functions by

$$
\omega(\Phi)(x):=\omega(\phi(x)), \quad \phi \in \mathbf{T}_{\mathbf{0}},
$$

where $\Phi$ is the thermal function corresponding to the thermal observable $\phi(x)$ by 2.3 . We interpret $\omega(\Phi)(x)$ as the expectation value of the thermal function $\Phi$ in the LTE state $\omega$ at the point $x \in \mathbf{M}_{\mathbf{0}}$. This is a well defined linear functional, since by $\mathbf{T}_{\mathbf{0}}(x)$-thermality we have $\omega\left(\phi_{1}(x)\right)=\omega(\Phi)(x)=\omega\left(\phi_{2}(x)\right)$ if $\Phi$ is a thermal function corresponding to both $\phi_{1}(x)$ and $\phi_{2}(x)$. A situation where two distinct thermal observables give rise to the same thermal function marks the occurrence of an equation of state, see the comment after the proof of Proposition 2.1.3.

In order to consider states in which the thermal functions can vary over spacetime, we extend Definition 2.1.1 to states that are in LTE in a region $\mathcal{O}$ of Minkowski spacetime.

Definition 2.1.2. A state $\omega$ is called $\mathbf{T}_{\mathbf{0}}(\mathcal{O})$-thermal if it is $\mathbf{T}_{\mathbf{0}}(x)$-thermal for all $x \in \mathcal{O}$. 
The spatio-temporal behaviour of the thermal functions in the state $\omega$ is thus encoded in the functions $x \mapsto \omega(\Phi)(x)$ obtained via 2.5). In [BOR02] it is shown that the microscopic dynamics of the thermal observables may give rise to equations governing the spacetime dependence of the macroscopic observables.

\subsubsection{Existence of Local Thermal Equilibrium States}

In the following we wish to discuss the basic question whether there exist any non-trivial LTE states (apart from the obvious ones in $\mathcal{C}$ ). This issue has been adressed in [BOR02] already, where it was found that for finite sets of thermal observables $\mathbf{T}_{\mathbf{0}}$ there exist non-trivial $\mathbf{T}_{\mathbf{0}}(x)$-thermal states. Here, we extend this result and show that one can find $\mathbf{T}_{\mathbf{0}}(\mathcal{O})$-thermal states for any compact region $\mathcal{O}$ in Minkowski spacetime, cf. [Sol10].

We assume that $\mathbf{T}_{\mathbf{0}}$ is finite. For each point $x$ in Minkowski spacetime, we introduce vector spaces

$$
S(x):=\operatorname{span}\left\{\phi(x): \phi \in \mathbf{T}_{\mathbf{0}}\right\},
$$

which are finite-dimensional by assumption. Moreover, the $S(x)$ are generated by locally covariant quantum fields and hence $\alpha_{x} S(0)=S(x)$. This implies that all $S(x)$ are isomorphic as vector spaces.

On each of the spaces $S(x)$ we define the semi-norms

$$
\tau_{B}(\phi(x)):=\sup _{\beta \in B}\left|\omega_{\beta}(\phi(x))\right|
$$

for compact subsets $B \subset V^{+}$. Note that we denote each of these semi-norms by $\tau_{B}$, disregarding on which space $S(x)$ they are defined.

Proposition 2.1.3. Let $\mathcal{O}$ be a compact subregion of Minkowski spacetime. Assume that there is some compact $B \subset V^{+}$such that $\tau_{B}$ constitutes a norm on $S(0)$. Then, there exist $\mathbf{T}_{\mathbf{0}}(\mathcal{O})$-thermal states in $\mathcal{S}_{0}$ which are not in $\mathcal{C}$.

Proof. We pick any state $\omega_{0} \in \mathcal{S}_{0}$ with $\omega_{0} \notin \mathcal{C}$. In the following, we use $\omega_{0}$ to construct an LTE state $\omega$ that is not in the set of reference states $\mathcal{C}$.

Since $\tau_{B}$ is a norm on $S(0)$, it is also on each $S(x), x \in \mathcal{O}$. Both this and the finitedimensionality of the $S(x)$ imply that for each $x \in \mathcal{O}$ there is a positive real number $C_{x}$ such that

$$
\left|\omega_{0}(\phi(x))\right| \leq C_{x} \tau_{B}(\phi(x)) \text { for all } \phi(x) \in S(x) .
$$

This in turn implies that for each $x \in \mathcal{O}$ we can lift $\omega_{0}$ to become a linear functional on the subspace of the thermal functions corresponding to $\mathbf{T}_{\mathbf{0}}$ by 2.3 . This is done by setting $\omega_{0}(\Phi)(x):=\omega_{0}(\phi(x)$ ), where $\Phi$ is the thermal function corresponding to $\phi(x)$ (cf. (2.3)). In particular $\Phi \equiv 0$ entails $\omega_{0}(\Phi)(x)=0$, so the (normalized) functionals $\omega_{0}(\cdot)(x)$ 
are well-defined for each $x \in \mathcal{O}$. Moreover, they are bounded by the norm $\tau_{B}$ and thus we may extend them to the space of continuous complex-valued functions on $B, C^{0}(B)$, by use of the Hahn-Banach theorem. We choose these extensions, denoted by $\omega_{0}(\cdot)(x)$ once again, to be Hermitian. For each $x \in \mathcal{O}$ we find

$$
\left|\omega_{0}(F)(x)\right| \leq C_{x}\|F\|_{B} \text { for all } F \in C^{0}(B)
$$

where $\|\cdot\|_{B}$ is the supremum norm on $C^{0}(B)$, with respect to which this space is a commutative $C^{*}$-algebra with unit. Any normalized Hermitian linear functional on this algebra can be represented by a normalized signed measure $\sigma_{x}$ [Coh80], decomposable into two positive measures: $\sigma_{x}=\sigma_{x}^{+}-\sigma_{x}^{-}$. Thus, for each $x \in \mathcal{O}$,

$$
\omega_{0}(F)(x)=\int_{B} d \sigma_{x}(\beta) F(\beta) .
$$

Assume that we are given a positive measure $\tau$ such that the sum $\sigma_{x}+\tau$ is a positive measure for all $x \in \mathcal{O}$ and let us define the state

$$
\omega:=(1+\tau(B))^{-1}\left(\omega_{0}+\int_{B} d \tau(\beta) \omega_{\beta}\right) .
$$

It is easy to see that for any $x \in \mathcal{O}$ and for all $\phi(x) \in S(x)$, we have $\omega(\phi(x))=$ $\int_{B} d \rho_{x}(\beta) \omega_{\beta}(\phi(x))$ with measure

$$
\rho_{x}:=(1+\tau(B))^{-1}\left(\sigma_{x}+\tau\right) .
$$

In other words, $\omega$ is a $\mathbf{T}_{\mathbf{0}}(x)$-thermal state for all $x \in \mathcal{O}$, i.e. $\omega$ is $\mathbf{T}_{\mathbf{0}}(\mathcal{O})$-thermal. It is not a reference state in general, since $\omega_{0}$ is not.

It remains to construct a suitable measure $\tau$. We have obtained the functionals $\omega_{0}(\cdot)(x)$ for each $x$ on the space of thermal functions corresponding to elements of $S(0)$. However, $S(0)$ is a finite dimensional vector space and hence we may express each of the $\omega_{0}(\cdot)(x)$ in terms of a finite basis of Hermitian functionals $\left\{\omega^{i}\right\}_{i=1}^{n}$ :

$$
\omega_{0}(\cdot)(x)=\sum_{i=1}^{n} a_{i}(x) \omega^{i}
$$

for some $a_{i}(x) \in \mathbb{R}$. Due to the continuity of $x \mapsto \omega_{0}(\phi(x)$ ) for all $\phi(x) \in S(x)$ (which is guaranteed by our choice of $\left.\mathcal{S}_{0}\right)$, we find that $x \mapsto \omega_{0}(\Phi)(x)$ is continuous for all thermal functions $\Phi$ corresponding to $\mathbf{T}_{\mathbf{0}}(x)$. The coefficient functions $a_{i}: \mathcal{O} \rightarrow \mathbb{R}$ are therefore continuous and, by compactness of $\mathcal{O}$, bounded by positive constants $C_{i}$. Again, we may use the Hahn-Banach theorem to extend the functionals $\omega^{i}$ to Hermitian functionals on all of $C^{0}(B)$, which we also denote by $\omega^{i}$. As before, each of the $\omega^{i}$ corresponds to a signed measure $\sigma^{i}=\sigma^{i,+}-\sigma^{i,-}$ and it follows that the measures $\sigma_{x}$ in 2.9 can be written as 
$\sigma_{x}=\sum_{i=1}^{n} a_{i}(x) \sigma^{i}$. Therefore, the $x$-dependence of the signed measures $\sigma_{x}$ is contained in the functions $a_{i}$. We now define the positive measure

$$
\tau:=\sum_{i=1}^{n} C_{i}\left|\sigma^{i}\right|,
$$

where $\left|\sigma^{i}\right|:=\sigma^{i,+}+\sigma^{i,-}$ denote the variations of the signed measures $\sigma^{i}$, which are by definition positive. It is easy to see that $\sigma_{x}+\tau$ is a positive measure for all $x \in \mathcal{O}$. This concludes the proof.

The LTE states we have constructed are of the form indicated in 2.10 and may therefore be interpreted as a "perturbation" (given by $\omega_{0}$ ) around the thermal reference state $\frac{1}{\tau(B)} \int_{B} d \tau(\beta) \omega_{\beta}$ with appropriate measure $\tau$. In these states, the thermal functions corresponding to $\mathbf{T}_{\mathbf{0}}$ will exhibit non-trivial spacetime dependence as may be seen by the fact that $\omega(\Phi)(x)=\omega_{\rho_{x}}(\phi(0))$ for all $x \in \mathcal{O}$. The measure $\rho_{x}$, given by equation 2.11, clearly depends non-trivially on $x$, because the function $x \mapsto \omega_{0}(\phi(x))$ depends non-trivially on $x$.

Let us comment on the assumptions we made. As was shown in [BOR02, the fact that $\tau_{B}$ is a norm on the spaces $S(x)$ is equivalent to non-existence of linear constraints emerging from equations of state, i.e. non-existence of linear relations between the thermal functions corresponding to $\mathbf{T}_{\mathbf{0}}$. To see this more clearly, note that if $\tau_{B}$ is a norm, it follows that $\phi(x)=0$ whenever the corresponding thermal function $\Phi$ vanishes on $B$ : $\Phi(\beta)=0 \forall \beta \in B$. Therefore there are no linear relations between the thermal functions that are not found among the fields themselves and hence no linear equations of state. Note that equations of state typically impose non-linear relations between the basic thermal functions and therefore do not present an obstruction in the sense discussed here 3

It is shown in [BOR02] that the argument for the existence of $\mathbf{T}_{\mathbf{0}}(x)$-thermal states at one point $x$ can be extended to the case where $\tau_{B}$ is only a semi-norm, which then covers systems with linear equations of state. However, it is not clear whether the same reasoning may be adopted in case of $\mathbf{T}_{\mathbf{0}}(\mathcal{O})$-thermality.

This, in fact, also presents a difficulty in exhibiting the existence of LTE states in regions of curved spacetime, as shown in the next section. It turns out that - for a small set of thermal observables for the free scalar field - linear equations of state yield non-trivial dynamical constraints on LTE states. Currently, we lack a method to show existence of states satisfying these conditions. However, there are examples of LTE states for linear equations of state in curved spacetime (see Chapter 3), so in principle this is no obstruction.

\footnotetext{
${ }^{3}$ Think of the Stefan-Boltzmann law, for example, which will play a rôle in Chapter 3.
} 


\subsection{Local Thermal Equilibrium States in Curved Spacetime}

In this section, we discuss the definition of LTE states for quantum field theory in curved spacetime. As was described in the introduction, the main motivation for this is that we would like to be able to attach thermal parameters to states of interest, for example in cosmological models. In the presence of curvature, this directly leads to the concept of local equilibrium, since there are no KMS states on a general curved spacetime $\mathbf{M}$ and hence no obvious candidates for states that represent ensembles which are in thermal equilibrium. This also leads to problems when trying to define LTE, unlike in Minkowski spacetime, as there are no reference states in $\mathcal{S}(\mathbf{M})$.

\subsubsection{Definition of Local Equilibrium in Curved Backgrounds}

As before, we assume that there is a lcQFT $\mathcal{A}$ with locally covariant state space $\mathcal{S}$ such that in Minkowski spacetime, $\mathcal{S}_{0}$ includes a set $\mathcal{C}$ of reference states which satisfies the assumptions laid down in Subsection 2.1.1. Moreover, in Minkowski spacetime, let there be a finite set of thermal observables

$$
\mathbf{T}_{\mathbf{0}}:=\left\{\phi_{0}^{(1)}, \ldots, \phi_{0}^{(n)}\right\}
$$

It follows from the discussion in the previous section that it is meaningful to define a state $\omega_{0} \in \mathcal{S}_{0}$ to be in LTE at $x \in \mathbf{M}_{\mathbf{0}}$, if the "vector" of expectation values

$$
\omega_{0}\left(\mathbf{T}_{\mathbf{0}}(x)\right):=\left(\omega_{0}\left(\phi_{0}^{(1)}(x)\right), \ldots, \omega_{0}\left(\phi_{0}^{(n)}(x)\right)\right) \in \mathbb{R}^{n}
$$

takes its values in a certain range, namely inside the convex set

$$
\mathfrak{T}_{\mathcal{C}}:=\left\{\left(\omega_{\rho}\left(\phi_{0}^{(1)}(x)\right), \ldots, \omega_{\rho}\left(\phi_{0}^{(n)}(x)\right)\right) \mid \omega_{\rho} \in \mathcal{C}\right\} \subset \mathbb{R}^{n} 4^{4}
$$

The basic strategy to define LTE in a curved background $\mathbf{M}$ is the same: one uses some set of point-like thermal observables and records their expectation values in a given state $\omega \in \mathcal{S}(\mathbf{M})$ in order to test whether the vector of their values lies within a specified range $\mathfrak{R} \subset \mathbb{R}^{n}$. Since the local thermal observables in Minkowski spacetime are sensitive to the thermal properties of the states, it seems natural to use their curved spacetime counterparts for the definition of LTE in M. In plain terms, if $\phi_{0}=\phi_{\mathbf{M}_{\mathbf{0}}}$ is a thermal observable in Minkowski spacetime, we use $\phi_{\mathbf{M}}$ as a thermal observable in $\mathbf{M}$. The

\footnotetext{
${ }^{4}$ This point of view makes it clear why linear equations of state have an effect on proofs of existence of LTE states - in contrast to non-linear equations of state, they reduce the convex dimension of $\mathfrak{T}_{\mathcal{C}}$. While one may find a state with vector of expectation values in $\mathfrak{T}_{\mathcal{C}}$ at some $x$, one also needs to make sure that for points close to $x$, the vector of expectation values stays in $\mathfrak{T}_{\mathcal{C}}$. But this is not guaranteed even by continuity, since $\mathfrak{T}_{\mathcal{C}}$ is not open.
} 
physical picture behind this is that our measuring devices are gauged in regions far away from any sources of gravity, where spacetime is essentially flat, and then moved to the region in which we want to probe, which may be curved.

Therefore, what matters are the locally covariant quantum fields $\phi$, i.e. the assignments $\mathbf{M} \mapsto \phi_{\mathrm{M}}$ (cf. Definition 1.1.9). However, in general, there are many choices for $\phi$ such that $\phi_{\mathrm{M}_{0}}$ equals a given quantum field $\phi_{0}$. Hence there is some leeway in the determination of the thermal observables in curved spacetime. This will become apparent when we deal with the scalar field in Chapter 3.

Since there are no reference states in $\mathcal{S}(\mathbf{M})$, the next question is what the set $\mathfrak{R}$ should be. Buchholz and Schlemmer [BS07] argue that since thermal observables are gauged in flat spacetime, one should choose $\mathfrak{R}=\mathfrak{T}_{\mathcal{C}}$. This means that we distinguish LTE states in curved spacetime by requesting that they respond like thermal reference states in Minkowski spacetime when tested with thermal observables.

For notational simplicity, let us assume that the fields $\phi^{(i)}$ in $\mathbf{T}$ correspond to contravariant tensors of rank $r(i)$. For $x \in \mathbf{M}$, we define the set $\mathbf{T}(x)$ to consist of all the components of the fields $\phi_{\mathbf{M}}^{(i)}, i=1, \ldots, n$, in some local Lorentz frame $\left\{e_{\mu}(x)\right\}$ at $x$. At the origin 0 of Minkowski spacetime $\mathbf{M}_{\mathbf{0}}$, we thus also have the set $\mathbf{T}_{0}(0)$, defined with respect to some Lorentz frame $\left\{e_{\mu}(0)\right\}$. For the comparison of measurements performed at $x \in \mathbf{M}$ and at $0 \in \mathbf{M}_{\mathbf{0}}$, we have to identify the tangent spaces at these points, which is done by identification of the frames $\left\{e_{\mu}(x)\right\}$ and $\left\{e_{\mu}(0)\right\}$. More specifically, we identify $\left(\varphi_{x}\right)_{*} \phi_{\mathbf{M}}$ with $\left(\varphi_{0}\right)_{*} \phi_{\mathbf{M}_{0}}$, where $\varphi_{x}:=e_{x} \circ \exp _{x}^{-1}$ are normal coordinates on some geodesic normal neighbourhood $\mathcal{O} \subset M$ of $x$, with $e_{x}$ being the isomorphism between $\exp _{x}^{-1}(\mathcal{O})$ and its image, afforded by $\left\{e_{\mu}(x)\right\}$ (similarly for $\varphi_{0}$ and $\left\{e_{\mu}(0)\right\}$ ).

Definition 2.2.1. A state $\omega \in \mathcal{S}(\mathbf{M})$ is $\mathbf{T}(x)$-thermal if for some reference state $\omega_{\rho_{x}} \in \mathcal{C}$ it holds that

$$
\omega\left(\phi_{\mathbf{M}}^{(i)}, \mu_{1} \ldots \mu_{r(i)}(x)\right)=\omega_{\rho_{x}}\left(\phi_{\mathbf{M}_{\mathbf{0}}}^{(i)}, \mu_{1} \ldots \mu_{r(i)}(0)\right)
$$

for all $\mu_{1}, \ldots, \mu_{r(i)}$ for all $i=1, \ldots, n$.

Using the identification of $\left(\varphi_{x}\right)_{*} \phi_{\mathbf{M}}$ with $\left(\varphi_{0}\right)_{*} \phi_{\mathbf{M}_{\mathbf{0}}}$ in the geodesic normal set $\mathcal{O}$, we can extend the definition of LTE to regions. Thus we say that $\omega \in \mathcal{S}(\mathbf{M})$ is $\mathbf{T}(\mathcal{O})$-thermal if it is $\mathbf{T}(y)$-thermal for all $y \in \mathcal{O}$ with respect to this identification of frames.

Thermal functions can now be attached to an $\mathbf{T}(x)$-thermal state $\omega$ as in Minkowski spacetime, i.e.

$$
\omega\left(\Phi_{\mu_{1} \ldots \mu_{r(i)}}\right)(x):=\omega\left(\phi_{\mathbf{M}, \mu_{1} \ldots \mu_{r(i)}}^{(i)}(x)\right),
$$

where $\Phi$ is the thermal function corresponding to $\phi_{\mathbf{M}_{\mathbf{0}}}=\phi_{0}$ by equation 2.3 .

The identification of the two frames $\left\{e_{\mu}(x)\right\}$ and $\left\{e_{\mu}(0)\right\}$ by use of normal coordinates is arbitrary. The definition of LTE is not affected by this choice, as the following example 
illustrates. Let $\omega \in \mathcal{S}(\mathbf{M})$ be in LTE at $x$ with regard to some thermal observable $\phi_{\mathbf{M}, \mu}(x)$ under the identification of $\left\{e_{\mu}(x)\right\}$ and $\left\{e_{\mu}(0)\right\}$. For simplicity, suppose $\omega$ has a definite temperature vector $\beta \in V^{+}$at $x$. This means

$$
\omega\left(\phi_{\mathbf{M}, \mu}(x)\right)=\omega_{\beta}\left(\phi_{\mathbf{M}_{0}, \mu}(0)\right) .
$$

Choosing another frame $e^{\prime}(x)=e(x) \Lambda^{-1}$ (where $\Lambda$ is some (proper) local Lorentz transformation of $\left.T_{x} M\right)$, we find

$$
\begin{aligned}
\omega\left(\phi_{\mathrm{M}, \mu^{\prime}}(x)\right) & =\left(\Lambda^{-1}\right)_{\mu^{\prime}}^{\mu} \omega\left(\phi_{\mathbf{M}, \mu}(x)\right) \\
\stackrel{2.14}{-} & \left(\Lambda^{-1}\right)^{\mu}{ }_{\mu^{\prime}} \omega_{\beta}\left(\phi_{0, \mu}(0)\right) \\
= & \omega_{\Lambda \beta}\left(\phi_{0, \mu}(0)\right),
\end{aligned}
$$

where the last equality follows from the transformation law of the KMS states under the Poincaré group 2.1. Had we now identified $\left\{e_{\mu^{\prime}}^{\prime}(x)\right\}$ and $\left\{e_{\mu}(0)\right\}$, we would still conclude that $\omega$ is in LTE with the same scalar temperature $\beta \in \mathbb{R}^{+}$. However, the thermal function $\Phi_{\mu}$ corresponding to $\phi_{0, \mu}$ is transformed according to the transformation of the local frame in $\mathbf{M}$.

Observation 2.2.2. Using some local Lorentz frame $e(x)$, let $\omega \in \mathcal{S}(\mathbf{M})$ be $\mathbf{T}(x)$-thermal and let $\omega(\Phi)(x)$ be the local thermal function corresponding to some $\phi \in \mathbf{T}$. If $e(x) \Lambda^{-1}(x)$ is another frame, then $\omega$ is also $\mathbf{T}(x)$-thermal with respect to this new frame and the local thermal function corresponding to $\phi$ is $\omega\left(\Lambda^{*} \Phi\right)(x)$.

This is the local equivalent of the transformation behaviour of global equilibrium states under Poincaré transformations of $\mathbf{M}_{\mathbf{0}}$.

It should also be noted here that in the context of curved spacetimes, it is not possible to work with vector spaces of thermal observables, as originally proposed in [BOR02] and [BS07]. As was pointed out in [Sol10], linear combinations of thermal observables are generally not thermal observables.

Let us illustrate this in the example of the massless, conformally coupled scalar field $P \phi:=\left(\square+\frac{1}{6} R\right) \phi=0$ (cf. Section 1.2.1, the thermostatics of which we treat in the next chapter. The set of locally covariant thermal observables which we use here is given by [SV08]

$$
\mathbf{T}^{(2)}:=\left\{\mathbb{1},: \phi^{2}:, \epsilon_{a b}\right\} .
$$

Here, the tensor $\epsilon_{a b}$ is the thermal energy tensor [BOR02, SV08], the components of which are given at $x \in \mathbf{M}$ by

$$
\epsilon_{\mu \nu}(x):=-: \phi\left(\nabla_{\mu} \nabla_{\nu} \phi\right):(x)+\frac{1}{4} \nabla_{\mu} \nabla_{\nu}: \phi^{2}:(x) 5^{5}
$$

\footnotetext{
${ }^{5}$ Note that, using the Leibniz rule, one can express this tensor in different ways.
} 
After choosing an identification of frames as described before, the condition of $\mathbf{T}^{(2)}(x)$ thermality of a state $\omega \in \mathcal{S}(\mathbf{M})$ is

$$
\begin{aligned}
& \omega\left(: \phi^{2}:(x)\right)=\omega_{\rho_{x}}\left(: \phi^{2}:_{0}(0)\right) \text { and } \\
& \omega\left(\epsilon_{\mu \nu}(x)\right)=\omega_{\rho_{x}}\left(\epsilon_{0, \mu \nu}(0)\right) \text { for } \mu, \nu=0,1,2,3
\end{aligned}
$$

for some thermal reference state $\omega_{\rho_{x}}$ in Minkowski spacetime.

At $x \in \mathbf{M}$, we have $\mathrm{f}^{6}$

$$
\epsilon_{\mu}^{\mu}(x)=\frac{1}{4} \square: \phi^{2}:(x)-\frac{1}{6} R: \phi^{2}:(x)+: \phi P \phi:(x) .
$$

It follows from the discussion at the end of Section 1.2 .3 that

$$
: \phi P \phi:(x)=U(x) \mathbb{1}
$$

with some smooth function $U$, which is determined by local curvature terms. This contribution is related to the conformal anomaly in equation 1.43, see Chapter 3 , equation 3.31, for its explicit form. Hence

$$
\epsilon_{\mu}^{\mu}(x)=\frac{1}{4} \square: \phi^{2}:-\frac{1}{6} R: \phi^{2}:(x)+U(x) \mathbb{1},
$$

yet at the origin of Minkowski spacetime the same linear combination yields

$$
\epsilon_{0}{ }_{\mu}^{\mu}(0)=\frac{1}{4} \square: \phi^{2}:_{0}(0) .
$$

Therefore, the locally covariant quantum field $\square: \phi^{2}$ :, which is actually a linear combination of the elements of $\mathbf{T}^{(2)}$, cannot itself be a thermal observable. This instead would require

$$
\omega\left(\square: \phi^{2}:(x)\right)=\omega_{\rho_{x}}\left(\square: \phi^{2}: 0(0)\right)=\square \omega_{\rho_{x}}\left(: \phi^{2}:{ }_{0}(0)\right)=0
$$

(by translation invariance of the reference state $\omega_{\rho_{x}}$ ) and thus by equations 2.18, 2.19) and $\mathbf{T}^{(2)}(x)$-thermality of $\omega$ it follows that $\frac{1}{6} R(x) \omega_{\rho_{x}}\left(: \phi^{2}:_{0}(0)\right)=U(x)$. Since the Wick square is, up to a constant, the mean of the local temperature squared, it follows that, at $x$, the expectation value of the local temperature takes a fixed value (determined by the ratio $U(x) / R(x))$ for all LTE states. This, of course, is not a sensible prediction.

Observation 2.2.3. The identification of $\mathbf{T}^{(2)}(x)$ and $\mathbf{T}^{(2)}(0)$, provided by the locally covariant quantum fields, cannot be extended to a linear map between $\operatorname{span}\left\{\mathbf{T}^{(2)}(x)\right\}$ and $\operatorname{span}\left\{\mathbf{T}^{(2)}(0)\right\}$.

\footnotetext{
${ }^{6}$ Recall that $\square: \phi^{2}:(x)=\eta^{\mu \nu} \nabla_{\mu} \nabla_{\nu}: \phi^{2}:(x)$, since the frame is taken to be orthonormal at $x$. Here, $\eta^{\mu \nu}$ are the entries of the diagonal matrix $\operatorname{diag}(-1,1,1,1)$.
} 
This would require the term $U(x) \mathbb{1}-\frac{1}{6} R(x): \phi^{2}:(x)$ to vanish, which it clearly does not. If we accept, however, that $\square: \phi^{2}$ : is not a thermal observable, we see that the condition of $\mathbf{T}^{(2)}(x)$-thermality does not require it to take the same value in $\omega$ as $\square: \phi^{2}:(0)$ does in the reference state $\omega_{\rho}$ (i.e. zero), but by equations 2.18, 2.19) and $\mathbf{T}^{(2)}(x)$-thermality the expectation value $\omega\left(\square: \phi^{2}:(x)\right)$ is fixed by

$$
\frac{1}{4} \omega\left(\square: \phi^{2}:(x)\right)-\frac{1}{6} R \omega_{\rho_{x}}\left(: \phi^{2}: 0(0)\right)+U(x) \mathbb{1}=0 .
$$

Therefore, the definition of LTE on curved spacetimes given in [BS07] has to be relaxed, by just considering sets of thermal observables instead of whole vector spaces 7 Note, however, that in some instances it may be possible to define thermal observables in curved spacetime whose linear relations among each other are of the same form as in Minkowski spacetime, in which case there can be a vector space structure on the set of thermal observables. See our discussion of the modified thermal energy tensor in Sections 3.1 and 3.2 for an example of this. In general, however, it is not clear whether such a choice is always possible and we tentatively assume that there is no additional structure on the sets of thermal observables.

Assuming that $\omega$ is $\mathbf{T}^{(2)}(\mathcal{O})$-thermal for some open region $\mathcal{O} \subset \mathbf{M}$, one can interpret equation 2.20 in the following way. Using the fact that

$$
\begin{aligned}
\omega\left(: \phi^{2}:(x)\right) & =\square_{x} \omega\left(: \phi^{2}:(x)\right) \\
& =\square_{x} \omega_{\rho_{x}}\left(: \phi^{2}: 0(0)\right)
\end{aligned}
$$

by $\mathbf{T}^{(2)}(x)$-thermality, we find the following differential equation that governs the behaviour of the function $x \mapsto \omega_{\rho_{x}}\left(: \phi^{2}:_{0}(0)\right)$ in $\mathcal{O}$ :

$$
\left(-\frac{1}{4} \square_{x}+\frac{1}{6} R(x)\right) \omega_{\rho_{x}}\left(: \phi^{2}: 0(0)\right)=U(x) .
$$

As we have mentioned before, the thermal function corresponding to the Wick square is up to a constant the temperature squared [BOR02].

Observation 2.2.4. The condition of LTE entails an evolution equation of Klein Gordon type for the mean of the square of the local temperature $T(x)$ of the system in $\mathcal{O}$ :

$$
\left(-\frac{1}{4} \square_{x}+\frac{1}{6} R(x)\right) \overline{T^{2}}(x) \sim U(x)
$$

This is one of the instances where in a LTE state the microscopic dynamics of the system yields an equation that governs the spacetime behaviour of a macroscopic observable. It

\footnotetext{
${ }^{7}$ Of course, the use of vector spaces of thermal observables is valid and equivalent to our definition in the particular case of Minkowski spacetime.
} 
is an interesting generalization of the corresponding equation in LTE states in Minkowski spacetime. We will discuss equation 2.22 in more detail in Chapter 3 , where we also propose a redefinition of the thermal energy tensor which also alters the form of 2.22 .

We find that linear constraints, arising from equations of state, in LTE states find a local analogue in evolution equations which depend on the spacetime geometry. I.e. the "local equations of state" depend on the background, which is something to be expected.

We have seen that relations among the microscopic observables (i.e. quantum fields) play an important rôle in the theory of local equilibrium. In particular, one has to be careful in the choice of of sets of thermal observables. Here, we introduce a criterion which we consider necessary for a consistent definition of LTE in curved spacetime. As before, we illustrate our point using the model of the Klein Gordon field, for simplicity taken to be massless and conformally coupled.

In [BOR02] it was explained that, in this model, the stress energy tensor $T_{a b}$ cannot be a thermal observable in Minkowski spacetime 8 . From the point of view of locally covariant quantum field theory, there is another reason why $T_{a b}$ cannot be a thermal observable. From equation 1.43 we see that

$$
g^{a b} T_{a b}=\left(-\frac{1}{4 \pi^{2}}\left[v_{1}\right]+g^{a b} T_{a b}^{R F}\right) \mathbb{1},
$$

which is the conformal anomaly. As explained before, the right hand side of this equation is in general non-vanishing in a curved spacetime $\mathbf{M}$, while it is identically zero in Minkowski spacetime $\mathbf{M}_{\mathbf{0}}$. Had we chosen $T_{a b}$ as a thermal observable, and were $\omega \in \mathcal{S}(\mathbf{M})$ to be in LTE with respect to this thermal observable in the sense of Definition 2.2.1, then at $x \in \mathbf{M}$ :

$$
\begin{aligned}
-\frac{1}{4 \pi^{2}}\left[v_{1}\right](x)+\eta^{\mu \nu} T_{\mu \nu}^{R F}(x) & =\eta^{\mu \nu} \omega\left(T_{\mu \nu}(x)\right) \\
& =\eta^{\mu \nu} \omega_{\rho}\left(T_{0 \mu \nu}(0)\right) \\
& =0,
\end{aligned}
$$

which is a contradiction - meaning that there cannot be any states in LTE with respect to $T_{a b}$.

The reason is that the set of observables consisting of $\mathbb{1}$ and $T_{a b}$ is not linearly independent on all spacetimes and the observable $\mathbb{1}$ is state independent. We therefore propose the following

Condition 1. The set of thermal observables $\mathbf{T}$ must be such that $\mathbf{T}(x)$ is linearly independent for all $x \in \mathbf{M}$ for all spacetimes $\mathbf{M}$.

\footnotetext{
${ }^{8} T_{a b}$ splits into two parts, one of which vanishes in all reference states. The other one is the thermal energy tensor $\epsilon_{a b}$, which is a thermal observable and was used in equation 2.16). See Chapter 3 for discussion.
} 
The set of thermal observables $\mathbf{T}^{(2)}$, cf. 2.15, satisfies this condition, because, as explained before, the field $\square: \phi^{2}$ : is not a thermal observable.

Another constraint on the set of thermal observables concerns quantum fields which contain covariant derivatives.

Observation 2.2.5. Thermal observables cannot contain covariant derivatives of order higher than two.

The reason is that, in Minkowski spacetime, partial derivatives commute, while they do not in the presence of curvature. It is easy to see how this leads to contradictions in the definition of local thermal equilibrium. It is reassuring that the thermal energy tensor (as most of the interesting observables in physics) does not contain spacetime derivatives of order higher than two.

To summarize: in a first step, we found that linear combinations of thermal observables need not be thermal observables again, which led us to adopt Definition 2.2.1 as the characterization of LTE in curved spacetime. In a second step, we argued that linear combinations of thermal observables cannot be thermal observables. The latter point seems to be of relevance in choosing "good" sets of thermal observables in any model.

It should be mentioned that our redefinition of LTE in curved spacetime does not affect results in previous publications on this subject, e.g. [SV08], where energy inequalities for LTE states in curved spacetime are discussed (see also [Sch10]).

\subsubsection{Existence of Local Equilibrium in Curved Backgrounds}

In the following, we present our results on the existence of pointwise LTE states in curved spacetime. The results naturally also apply to the special case of Minkowski spacetime. However, the methods used here are unrelated to the ones employed in Section 2.1.2 and the discussion relies on entirely different features of QFT. For notational reasons we restrict attention to the case when our sets of thermal observables consist only of scalar fields. However, the whole discussion, as well as the results that follow, also apply to more general tensor fields.

Given a set of locally covariant thermal observables $\mathbf{T}=\left\{\phi^{(i)}\right\}_{i=0}^{n}$ with $\phi^{(0)}=\mathbb{1}$, we define, for each spacetime $\mathbf{M}$, the following set of expectation values of $\mathbf{T}(x)$ at $x \in \mathbf{M}$ :

$$
\mathfrak{T}(x):=\left\{\left\{\omega\left(\phi^{(i)}(x)\right)\right\}_{i=1}^{n} \mid \omega \in \mathcal{S}(\mathbf{M})\right\} .
$$

$\mathfrak{T}(x)$ is a convex subset of $\mathbb{R}^{n}$, as is the set $\mathfrak{T}_{\mathcal{C}}$ of expectation values in the reference states $\mathcal{C}$ of $\mathbf{T}(0)$ at the origin of Minkowski spacetime, cf. equation 2.12 . Clearly $\mathfrak{T}(x) \cap \mathfrak{T}_{\mathcal{C}} \neq \emptyset$ is necessary and sufficient for the existence of $\mathbf{T}(x)$-thermal states. 
Let us briefly comment on the structure of the convex sets $\mathfrak{T}(x)$ and $\mathfrak{T}_{\mathcal{C}}$. The convex dimension ${ }^{9}$ of $\mathfrak{T}(x)$ equals $n$ because the thermal observables are linearly independent. Therefore, $\mathfrak{T}(x)$ has non-empty interior in $\mathbb{R}^{n}$. It is more complicated to determine the dimension of $\mathfrak{T}_{\mathcal{C}}$, but it is certainly less than $n$ when there are linear equations of state, i.e. linear relations among the thermal observables that show up in all reference states.

Because one expects quantum fields to be unbounded quantities, we show in Proposition 2.2.7 that it is physically meaningful to assume that $\mathfrak{T}(x)=\mathbb{R}^{n}$, i.e. that for every $n$-tuple of real numbers there are states in $\mathcal{S}(\mathbf{M})$ whose expectation values in the members of $\mathbf{T}(x)$ yield exactly this tuple.

Observation 2.2.6. If $\mathfrak{T}(x)=\mathbb{R}^{n}$, there exist states in $\mathcal{S}(\mathbf{M})$ that are $\mathbf{T}(x)$-thermal.

In the following, the assumption $\mathfrak{T}(x)=\mathbb{R}^{n}$ is related to properties of lcQFT that can be checked in examples, see Chapter 3 . For this, we define the numerical range of any element $\psi \in \operatorname{span}\{\mathbf{T}(x)\}$ to be the following subset of $\mathbb{R}:\{\omega(\psi) \mid \omega \in \mathcal{S}(\mathbf{M})\}$.

Proposition 2.2.7. The following two statements are equivalent.

1. The numerical range of any element in $\operatorname{span}\{\mathbf{T}(x)\}$ which is not a multiple of the identity equals all of $\mathbb{R}$.

2. $\mathfrak{T}(x)=\mathbb{R}^{n}$.

Proof. Without loss of generality, we assume that $\mathbf{T}(x)$ does not contain the unit $\mathbb{1}$. The following argument appears in the proof of [HW10, Theorem 5]. We will repeat it here for the convenience of the reader, filling in some details.

Item $1 \Rightarrow \mathfrak{T}(x)=\mathbb{R}^{n}$ : we argue by contradiction. Assume that $\mathfrak{T}(x)$ is a proper subset of $\mathbb{R}^{n}$. As we noted previously, $\mathfrak{T}(x)$ has non-empty interior in $\mathbb{R}^{n}: \mathfrak{T}(x)^{\circ} \neq \emptyset$.

We distinguish two cases. The first is that even the closure of the convex set $\mathfrak{T}(x)$ is a proper subset of $\mathbb{R}^{n}$. In this case, we can find a support hyperplane (an affine space of dimension $n-1$ that touches the boundary of $\mathfrak{T}(x))$ which may be written as $\left\{v \in \mathbb{R}^{n} \mid L(v)=\alpha\right\}$ for some linear functional $L$ on $\mathbb{R}^{n}$ and some $\alpha \in \mathbb{R}$. If the components of $L$ in the standard basis of $\mathbb{R}^{n}$ are given by $\left(l_{1}, \ldots, l_{n}\right)$, we find:

$$
l_{1} \omega\left(\phi^{(1)}(x)\right)+\cdots+l_{n} \omega\left(\phi^{(n)}(x)\right) \leq \alpha
$$

for all $\omega \in \mathcal{S}(\mathbf{M})$. Hence, the numerical range of $l_{1} \phi^{(1)}(x)+\cdots+l_{n} \phi^{(n)}(x)$ cannot be all of $\mathbb{R}$ - a contradiction.

\footnotetext{
${ }^{9}$ The dimension of a convex set is defined as the dimension of its affine hull. Recall that the affine hull of a subset $E$ of $\mathbb{R}^{n}$ is defined as the intersection of all affine subspaces of $\mathbb{R}^{n}$ containing $E$. See e.g. Hör94 for details.
} 
The other case is when the closure of $\mathfrak{T}(x)$ is all of $\mathbb{R}^{n}$, i.e. when $\mathfrak{T}(x)$ is dense in $\mathbb{R}^{n}$. We show that, in this case, $\mathfrak{T}(x)$ is already all of $\mathbb{R}^{n}$. Assume the contrary and take an element $a \in \mathbb{R}^{n}$ such that $a \notin \mathfrak{T}(x)$. As $\mathfrak{T}(x)$ has non-empty interior, we may choose an open subset $O \subset \mathfrak{T}(x)$. Then $a-(O-a)$ is also open and contains some $d \in \mathfrak{T}(x)$, because $\mathfrak{T}(x)$ is dense. But then $d=a-(b-a)$ for some $b \in O$ and hence $a=\frac{1}{2}(d+b)$. Since $b, d \in \mathfrak{T}(x)$ by construction, we conclude that $a \in \mathfrak{T}(x)$ due to convexity - a contradiction.

The other direction, $\mathfrak{T}(x)=\mathbb{R}^{n} \Rightarrow$ Item 1 , is obvious.

To summarize: when the set of thermal observables spans a space of "unbounded" linear forms, there exist states which are in LTE with regard to these observables. The unboundedness of the point-like observables makes sense physically, because it ensures that these quantum fields cannot be realized as proper observables (i.e. self-adjoint operators in Hilbert space), which is expected due to the uncertainty relations.

Concerning the existence of LTE in regions of curved spacetime, note the following. Suppose we have established $\mathbf{T}(x)$-thermality of a state $\omega$ at $x \in \mathbf{M}$. In the notation of the beginning of this section, this means that the vector of expectation values

$$
\omega(\mathbf{T}(x)):=\left(\omega\left(\phi^{(1)}(x)\right), \ldots, \omega\left(\phi^{(n)}(x)\right)\right) \in \mathbb{R}^{n}
$$

is an element of the set $\mathfrak{T}_{\mathcal{C}}=\left\{\left\{\omega_{\rho}\left(\phi_{0}^{(i)}(0)\right)\right\}_{i=1}^{n} \mid \omega_{\rho} \in \mathcal{C}\right\}$, cf. equation 2.12. Recall that it was one of our assumptions on the state space that for each $\omega \in \mathcal{S}(\mathbf{M})$ the map $x \mapsto \omega\left(\phi^{(i)}(x)\right)$ is at least continuous. If the set $\mathfrak{T}_{\mathcal{C}}$ has non-empty interior and we assume that $\omega(\mathbf{T}(x))$ lies in it, by continuity we may conclude that there is a neighbourhood $\mathcal{O}_{x}$ of $x$ such that $\omega$ is $\mathbf{T}\left(\mathcal{O}_{x}\right)$-thermal. However, if there are linear equations of state, it is easy to see that the convex dimension of $\mathfrak{T}_{\mathcal{C}}$ is strictly smaller than $n$ and thus even the smallest displacement of $x$ can push $\omega(\mathbf{T}(x))$ out of $\mathfrak{T}_{\mathcal{C}}$ and hence $\omega$ out of local thermal equilibrium.

Let us again illustrate this for the set of thermal observables $\mathbf{T}^{(2)}$ for the massless, conformally coupled free scalar field, cf. equation 2.15). As was already noted in equation 2.19, in Minkowski spacetime $\mathbf{M}_{\mathbf{0}}$ we have

$$
\epsilon_{0}{ }_{\mu}{ }_{\mu}(x)=\frac{1}{4} \square: \phi^{2}: 0(x)
$$

and by translational invariance of the reference states it follows that there is the following linear equation of state

$$
E^{\mu}{ }_{\mu}(\beta)=0, \beta \in V^{+},
$$

where $E_{\mu \nu}$ is the thermal energy, i.e. the thermal function corresponding to the thermal observable $\epsilon_{\mu \nu}$ by equation 2.3. This means that the dimension of the corresponding 
convex set $\mathfrak{T}_{\mathcal{C}}$ is lower than maximal and $\mathfrak{T}_{\mathcal{C}}$ cannot have non-empty interior. As was shown in equation 2.21), one instead gets a non-trivial constraint for $\mathbf{T}^{(2)}$-thermal states $\omega$ which takes the form of a Klein Gordon type equation for the expectation value of the Wick square. If $\omega$ is $\mathbf{T}^{(2)}(x)$-thermal and it satisfies this equation in some region $\mathcal{O} \subset \mathbf{M}$, then $\omega\left(\mathbf{T}^{(2)}(x)\right) \in \mathfrak{T}_{\mathcal{C}}$ for $x$ in some neighbourhood $\mathcal{O}_{x} \subset \mathcal{O}$ and $\omega$ is $\mathbf{T}\left(\mathcal{O}_{x}\right)$-thermal.

In [Sch10], Schlemmer has constructed $\mathbf{T}^{(2)}(\Sigma)$-thermal Hadamard states for a Cauchy surfaces $\Sigma$ in Robertson-Walker spacetimes with flat spatial sections, which shows that consistent initial conditions can be found for the dynamical constraint 2.21). However, the problem of existence of $\mathbf{T}^{(2)}$-thermal states in open regions remains unsolved and we do not pursue this question any further here.

\subsubsection{Unboundedness and Scaling Limits}

In the following, we wish to discuss the unboundedness of quantum fields in order to get a deeper understanding of condition 1 in Proposition 2.2.7. In QFT in Minkowski spacetime, it was shown that negative expectation values for the energy density are possible in Wightman theories [EGJ65]. Here, we review an extension of this argument given in [Few05]. It shows that if there is a scaling limit (with positive dimension) for the quantum field in question, the point-like analogue of this field is indeed an unbounded sesquilinear form. Applying this to a set of thermal observables $\mathbf{T}$, we see that the existence of $\mathbf{T}(x)$ thermal states can be proved if a suitable scaling limit for all elements of $\operatorname{span}\{\mathbf{T}(x)\}$ can be established, cf. Proposition 2.2.7. In Chapter 3, we show that for the free scalar field, certain sets of thermal observables $\mathbf{T}$ indeed span unbounded sesquilinear forms and hence there exist many $\mathbf{T}(x)$-thermal states at each $x \in \mathbf{M}$.

We need to recall the concept of scaling limits in the sense of [FH87], following [Few05]. For notational simplicity once again we deal with scalar fields only, but the definition may be generalized to include vector valued quantum fields without further conceptual difficulties [SV01]. Let $x \in \mathbf{M}$ and let $(U, \kappa)$ be a chart around $x$ such that $\kappa(U) \subset \mathbb{R}^{n}$ is convex and $\kappa(x)=0$. One defines a semi-group $\left\{\sigma_{\lambda}\right\}_{\lambda \in(0,1)}$ of local diffeomorphisms of $U$ by:

$$
\sigma_{\lambda}(y):=\kappa^{-1}(\lambda \cdot \kappa(y)) \text { for } y \in U \text { and } \lambda \in(0,1) .
$$

Clearly these maps contract $U$ to the point $x$ if $\lambda \rightarrow 0^{+}$. Their action on test functions on the $n$-th Cartesian power of $U$ is defined by:

$$
\left(\sigma_{\lambda *} f\right)\left(y_{1}, \ldots, y_{n}\right):=f\left(\sigma_{\lambda}^{-1}\left(y_{1}\right), \ldots, \sigma_{\lambda}^{-1}\left(y_{n}\right)\right)
$$

for all $\left(y_{1}, \ldots, y_{n}\right) \in U^{\times n}$ and $f \in \mathcal{D}\left(U^{\times n}\right)$. We set $\sigma_{\lambda *} f \equiv 0$ outside of $U$.

Definition 2.2.8. Given a quantum field $\phi$, consider the corresponding hierarchy of $n$ point functions $\omega^{(n)} \in \mathcal{D}^{\prime}\left(M^{\times n}\right)$ of a state $\omega$ in $\mathcal{S}(\mathbf{M})$. $\omega$ has a scaling limit at $x \in \mathbf{M}$ for 
the field $\phi$ if there exists a monotone scaling function $N:(0,1] \rightarrow[0, \infty)$ such that

$$
\hat{\omega}^{(n)}\left(f^{(n)}\right):=\lim _{\lambda \rightarrow 0^{+}} N(\lambda)^{n} \omega^{(n)}\left(\sigma_{\lambda *} f^{(n)}\right)
$$

exists for all $n \in \mathbb{N}$ and all $f^{(n)} \in \mathcal{D}\left(U^{\times n}\right)$ and is non-vanishing for some $n$ and $f^{(n)}$.

Due to sequential completeness, $\hat{\omega}^{(n)}$ is a distribution in $\mathcal{D}^{\prime}\left(U^{\times n}\right)$. The scaling function $N$ satisfies the following relation for some $\alpha \in \mathbb{R}$ [FH87]:

$$
\lim _{\lambda^{\prime} \rightarrow 0^{+}} \frac{N\left(\lambda \lambda^{\prime}\right)}{N\left(\lambda^{\prime}\right)}=\lambda^{\alpha}
$$

The number $d:=4+\alpha$ is called the dimension of the field $\phi$ at $x$. If $d$ is strictly positive, $d>0$, it follows that [Few05, p. 11]:

$$
\lim _{\lambda \rightarrow 0^{+}} \lambda^{4} N(\lambda)=0
$$

In the following, we consider the case that $\omega$ has a scaling limit for $\phi$ at $x \in M$ with strictly positive dimension such that the scaling limit two-point function $\hat{\omega}^{(2)}$ is non-trivial, i.e. does not vanish for all test functions in $\mathcal{D}(U \times U)$. We call such scaling limits positive.

Proposition 2.2.9 ([Few05]). Let $\phi$ be a quantum field in the GNS Hilbert space of some state $\omega \in \mathcal{S}(\mathbf{M})$ such that $\omega$ possesses a positive scaling limit for $\phi$ at $x \in \mathbf{M}$. It follows that the point-like localized field $\phi(x)$ is an unbounded sesquilinear form on the domain of the field.

Proof. We may assume that $\omega(\phi(f))=0$ for all $f \in \mathcal{D}(M) 10$ It is shown in [Few05, p. 11] that for positive scaling limits one finds an $f \geq 0$ in $\mathcal{D}(U)$ such that $\hat{\omega}^{(2)}(f \otimes f)>0$. We choose such an $f$ and keep it fixed in the following. It is then easy to see that we may find a sequence of real numbers $\left\{\lambda_{n}\right\}_{n \in \mathbb{N}} \subset(0,1]$ converging to zero such that

$$
\omega^{(2)}\left(\sigma_{\lambda_{n} *}(f \otimes f)\right)>0 \text { for all } n \in \mathbb{N} .
$$

We now put $f_{n}:=\lambda_{n}^{-4} \sigma_{\lambda_{n} *} f$. By 2.32 , we may define the following sequences of states: for each $n \in \mathbb{N}$ and each $c \in \mathbb{R}$ set $\omega_{c, n}(\cdot):=\omega\left(A_{n}(c) \cdot A_{n}(c)^{*}\right)$, with operators

$$
A_{n}(c):=\cos (c) \mathbb{1}+\sin (c) \frac{\phi\left(f_{n}\right)}{\omega^{(2)}\left(f_{n} \otimes f_{n}\right)^{\frac{1}{2}}} .
$$

Clearly $\omega_{c, n} \in \mathcal{S}(\mathbf{M})$ and it follows that

$$
\omega_{c, n}\left(\phi\left(f_{n}\right)\right)=a_{n} \sin (2 c)+b_{n}(1-\cos (2 c))
$$

\footnotetext{
${ }^{10}$ Otherwise we may apply the analysis to the shifted field $\phi(f)-\omega(\phi(f)) 1$, which does not change the conclusions, as one may easily check.
} 
where

$$
a_{n}:=\omega^{(2)}\left(f_{n} \otimes f_{n}\right)^{\frac{1}{2}} \text { and } b_{n}:=\frac{1}{2} \frac{\omega^{(3)}\left(f_{n} \otimes f_{n} \otimes f_{n}\right)}{\omega^{(2)}\left(f_{n} \otimes f_{n}\right)} .
$$

Minimizing expression 2.34 with regard to $c$ yields

$$
\inf _{\text {all states } \varphi} \varphi\left(\phi\left(f_{n}\right)\right) \leq a_{n}\left(\frac{b_{n}}{a_{n}}-\sqrt{1+\left(\frac{b_{n}}{a_{n}}\right)^{2}}\right)<0,
$$

where the infimum is taken over all states in the folium of $\omega$.

Let us now discuss the limit $n \rightarrow \infty$. Consider $a_{n}$ first: the scaling limit tells us that

$$
\lim _{n \rightarrow \infty}\left(\lambda_{n}^{4} N\left(\lambda_{n}\right) a_{n}\right)^{2}=\hat{\omega}^{(2)}(f \otimes f) \neq 0 .
$$

Using equation (2.31), we conclude that $a_{n} \rightarrow+\infty$ as $n \rightarrow \infty$. Secondly, considering the limit of the ratio $\frac{b_{n}}{a_{n}}$, we find

$$
\frac{b_{n}}{a_{n}}=\frac{N\left(\lambda_{n}\right)^{3} \omega^{(3)}\left(f_{n} \otimes f_{n} \otimes f_{n}\right)}{\left(N\left(\lambda_{n}\right)^{2} \omega^{(2)}\left(f_{n} \otimes f_{n}\right)\right)^{\frac{3}{2}}} \stackrel{n \rightarrow \infty}{\longrightarrow} \frac{\hat{\omega}^{(3)}(f \otimes f \otimes f)}{\left(\hat{\omega}^{(2)}(f \otimes f)\right)^{\frac{3}{2}}}
$$

by the scaling limit. This expression is finite. Thirdly, we note that upon proper normalization, $\left(f_{n}\right)_{n \in \mathbb{N}}$ is actually a delta sequence for $x \in \mathbf{M}: f_{n} \stackrel{n \rightarrow \infty}{\longrightarrow} \delta_{x}$.

Putting these three pieces of information together, it follows from equation 2.35 that

$$
\inf _{\text {all states } \varphi} \varphi(\phi(x))=-\infty,
$$

which just says that the sesquilinear form $\phi(x)$ is unbounded from below.

To derive unboundedness from above, one replaces $A_{n}(c)$ in 2.33 with

$$
B_{n}(c):=\cos (c) \mathbb{1}-\sin (c) \frac{\phi\left(f_{n}\right)}{\omega^{(2)}\left(f_{n} \otimes f_{n}\right)^{\frac{1}{2}}}
$$

and finds

$$
\sup _{\text {all states } \varphi} \varphi\left(\phi\left(f_{n}\right)\right) \geq a_{n}\left(-\frac{b_{n}}{a_{n}}+\sqrt{1+\left(\frac{b_{n}}{a_{n}}\right)^{2}}\right)>0 .
$$

Again, taking the limit $n \rightarrow \infty$ yields the desired result.

As we mentioned before, in order to use this result as a tool to establish unboundedness of all linear combinations of a particular space of thermal observables $\mathbf{T}(x):=\left\{\phi^{(i)}(x)\right\}_{i=1}^{n}$, it is necessary to conclude from the fact that the $\phi^{(i)}$ have positive scaling limits for all $i=1, \ldots, n$ that all linear combinations $\psi:=\sum_{i=1}^{n} c_{(i)} \phi^{(i)}$ also have positive scaling limits.

In general, however, there is a problem in the calculation of the scaling limit of the "mixed terms" appearing in the $m$-point functions of $\psi$ for $m>2$. A second difficulty 
appears if there are two or more fields in $\psi$ which share the highest scaling dimension. A priori, we have no means to exclude the possibility that there are certain cancellations in the limit which render the scaling limit two-point function of $\psi$ trivial.

Let us discuss the first problem. Suppose that a state $\omega$ has a positive scaling limit for each of the basic fields $\phi^{(i)}$ at $x$ with scaling function $N_{i}$ and dimension $d_{i}:=\alpha_{i}+4>0$. By Proposition 2.2.9 we know that the range of each of the $\phi^{(i)}(x)$ is all of $\mathbb{R}$. Let $\psi:=\sum_{i=1}^{n} c_{(i)} \phi^{(i)}$ be an arbitrary linear combination of these fields. In order to show that the field $\psi$ has a positive scaling limit at $x \in \mathbf{M}$, we need to investigate the scaling behaviour of its $m$-point functions

$$
\omega\left(\psi\left(x_{1}\right), \ldots, \psi\left(x_{m}\right)\right)=\sum_{i_{1}, \ldots, i_{m}} c_{\left(i_{1}\right)} \ldots c_{\left(i_{m}\right)} \omega\left(\phi^{\left(i_{1}\right)}\left(x_{1}\right) \ldots \phi^{\left(i_{m}\right)}\left(x_{m}\right)\right)
$$

where the $i_{1}, \ldots, i_{m}$ run from 1 to $n$. Suppose that among the $\phi^{(i)}$ there is one field which has the highest dimension at $x$, say $\phi^{(1)}$ with $c_{(1)} \neq 0$. We suspect that the scaling function of $\psi$ is $N_{1}$ and its dimension is $d_{1}$.

If $m=2$, this can be verified by use of the Cauchy Schwarz inequality:

$$
\begin{aligned}
& \left|N_{1}(\lambda)^{2} \omega\left(\psi\left(\sigma_{\lambda *} f\right) \psi\left(\sigma_{\lambda *} f\right)\right)\right| \leq \sum_{i_{1}, i_{2}} c_{\left(i_{1}\right)} c_{\left(i_{2}\right)}\left|N_{1}(\lambda)^{2} \omega\left(\phi^{\left(i_{1}\right)}\left(\sigma_{\lambda *} f\right) \phi^{\left(i_{2}\right)}\left(\sigma_{\lambda *} f\right)\right)\right| \\
& \leq \sum_{i_{1}, i_{2}} c_{\left(i_{1}\right)} c_{\left(i_{2}\right)}\left|N_{1}(\lambda)^{2} \omega\left(\phi^{\left(i_{1}\right)}\left(\sigma_{\lambda *} f\right) \phi^{\left(i_{1}\right)}\left(\sigma_{\lambda *} f\right)\right)\right|^{\frac{1}{2}}\left|N_{1}(\lambda)^{2} \omega\left(\phi^{\left(i_{2}\right)}\left(\sigma_{\lambda *} f\right) \phi^{\left(i_{2}\right)}\left(\sigma_{\lambda *} f\right)\right)\right|^{\frac{1}{2}} \\
& \stackrel{\lambda \rightarrow 0^{+}}{\longrightarrow} c_{(1)}^{2}\left|\hat{\omega}_{1}^{(2)}(f \otimes f)\right| .
\end{aligned}
$$

Here, $\hat{\omega}_{1}^{(2)}$ denotes the scaling limit two-point function for the field $\phi^{(1)}$ in the state $\omega$. In the last step, $\lambda \rightarrow 0^{+}$, we made use of the following result.

Lemma 2.2.10. Let $\phi^{(i)}, i=1,2$, be two quantum fields with respect to which a state $\omega$ possess a scaling limit at $x \in \mathbf{M}$ with scaling function $N_{i}$ and dimension $d_{i}:=\alpha_{i}+4$. Assume that $d_{1}>d_{2}$. Then for all $f^{(n)} \in \mathcal{D}\left(U^{\times n}\right)$

$$
\lim _{\lambda \rightarrow 0^{+}} N_{1}(\lambda)^{n} \omega_{2}^{(n)}\left(\sigma_{\lambda *} f^{(n)}\right)=0
$$

where the $\omega_{2}^{(n)} \in \mathcal{D}^{\prime}\left(M^{\times n}\right)$ are the $n$-point functions of $\omega$ for the field $\phi^{(2)}(f)$.

Proof. It is sufficient to show $\lim _{\lambda^{\prime} \rightarrow 0^{+}} \frac{N_{1}\left(\lambda^{\prime}\right)}{N_{2}\left(\lambda^{\prime}\right)}=0$. Note that it follows from equation 2.30 that for all $\lambda \in(0,1)$ there is a $\lambda_{i} \in(0,1]$ (depending on $\alpha_{i}$ and $\lambda$ ) such that

$$
\left(\frac{1}{2} \lambda^{\alpha_{i}}\right)^{n} N_{i}\left(\lambda^{\prime}\right) \leq N_{i}\left(\lambda^{n} \lambda^{\prime}\right) \leq\left(\frac{3}{2} \lambda^{\alpha_{i}}\right)^{n} N_{i}\left(\lambda^{\prime}\right)
$$

for all $0<\lambda^{\prime} \leq \lambda_{i}, i=1,2$. Choose $\lambda$ such that $3 \lambda^{\alpha_{1}-\alpha_{2}}<1$ (which is possible because of $\left.\alpha_{1}>\alpha_{2}\right)$, set $\lambda_{0}:=\min \left(\lambda_{1}, \lambda_{2}\right)$ and consider the sequence $\lambda_{n}:=\lambda^{n} \lambda_{0}$. For $0<\lambda^{\prime} \leq \lambda_{0}$, 
define the number $n\left(\lambda^{\prime}\right)$ by $\lambda^{\prime} \in\left[\lambda_{n\left(\lambda^{\prime}\right)+1}, \lambda_{n\left(\lambda^{\prime}\right)}\right)$. Clearly, $\lim _{\lambda^{\prime} \rightarrow 0^{+}} n\left(\lambda^{\prime}\right)=\infty$. We can now make use of the fact that the $N_{i}$ 's are monotonous functions. Assume, for example, that $N_{1}$ is monotonically increasing while $N_{2}$ is monotonically decreasing. Then, by 2.39, it follows that for all $0<\lambda^{\prime} \leq \lambda_{0}$ one has

$$
\frac{N_{1}\left(\lambda^{\prime}\right)}{N_{2}\left(\lambda^{\prime}\right)} \leq \frac{N_{1}\left(\lambda_{n\left(\lambda^{\prime}\right)}\right)}{N_{2}\left(\lambda_{n\left(\lambda^{\prime}\right)}\right)} \leq\left(3 \lambda^{\alpha_{1}-\alpha_{2}}\right)^{n\left(\lambda^{\prime}\right)} \frac{N_{1}\left(\lambda_{0}\right)}{N_{2}\left(\lambda_{0}\right)} \rightarrow 0
$$

as $\lambda^{\prime} \rightarrow 0^{+}$. The other cases can be treated in the same way.

In other words, when considering the scaling limit of a linear combination of fields $\psi$, in case of the two-point function $m=2$ one may suppress contributions from fields of lower dimension by using the scaling function of the field with highest dimension and only the scaling limit of this field survives. However, if $m \geq 3$, the same conclusion cannot be drawn so easily. In order to estimate the mixed terms appearing there, one cannot use the Cauchy Schwarz inequality in an obvious way. One therefore needs information on the scaling behaviour of products of the fields as in the case of Wick's theorem for the Wick products of the Klein Gordon field, cf. section 1.2.2. We will discuss this example in the next chapter.

As mentioned previously, if there are two or more distinct fields in $\mathbf{T}$ which have the same dimension at $x$, one needs to check carefully whether the scaling limit two-point functions of every possible linear combination of the two are still non-trivial, as cancellations in the scaling limit could occur. Examples where this may be a problem can be found among thermal observables which are different components of the same tensor, as is for the components of the thermal energy tensor for the free scalar field. 


\section{Local Thermal Equilibrium States for the Free Scalar Field}

In this chapter, we discuss local thermal equilibrium states for the free scalar field. In Section 3.1, we introduce the thermal observables we are dealing with, putting special emphasis on the thermal energy tensor and proposing a redefinition of this observable. Moreover, we consider higher Wick powers as thermal observables, finding that the renormalization freedom is greatly reduced if we introduce a natural condition concerning the combinatorics of quasi-free LTE states. We also introduce conformally covariant quantum fields at this stage, because later, we argue that the Wick square should be renormalized such that it becomes a conformally covariant quantum field in the sense of [Pin09]. This is justified by results for the Wick square in KMS states in certain simple stationary spacetimes, which we consider in Section 3.2. We also use our abstract results of Chapter 2 to show existence of LTE states in the model treated here.

\subsection{Basic Thermal Observables and Thermal Functions}

The concept of equilibrium makes reference to a notion of dynamics, which, in lcQFT, is implemented by one-parameter groups of time-like isometries, which give rise to automorphisms of the algebra of observables. While any KMS state with respect to such a one-parameter group of automorphisms is passive (i.e. no observer can extract energy from the state running a cyclic engine), it is only for global equilibrium states in Minkowski spacetime, defined with respect to inertial observers, that the KMS parameter $\beta$ has clear interpretation as an inverse temperature in the sense of the zeroth law of thermostatics. 1 This is why thermal observables are gauged exactly in these states.

On the other hand, one can use these thermal observables to test KMS states that are connected with certain time evolutions in curved spacetime. This may help to reveal the physical effects that give rise to passivity in these cases and, moreover, to elucidate the rôle of the KMS parameter and its relation to the local temperature in these states.

\footnotetext{
${ }^{1}$ Recall that temperature is a parameter that identifies macroscopic systems which are mutually in thermal equilibrium.
} 
In some cases, this allows one to draw conclusions about the relation between the local temperature and the Hawking-Unruh temperature, as we see later.

Definition 3.1.1. A spacetime $\mathbf{M}$ is called stationary if there exists a one-parametergroup of time-like isometries $\left\{\chi_{t}\right\}_{t \in \mathbb{R}}$ or, equivalently, if there is a time-like Killing vector field $K$ in $\mathbf{M}$. $\mathbf{M}$ is called static, if there is a family of space-like hypersurfaces orthogonal to $K$ everywhere: 2

In a lcQFT $\mathcal{A}$, the maps $\chi_{t}$ get lifted to automorphisms $\alpha_{t}$ on $\mathcal{A}(\mathbf{M})$. Hence, there exists a one-parameter-group of automorphisms $\left\{\alpha_{t}\right\}_{t \in \mathbb{R}}$ on $\mathcal{A}(\mathbf{M})$, which is interpreted as dynamics along the trajectories induced by $\left\{\chi_{t}\right\}_{t \in \mathbb{R}}$. With respect to this notion of dynamics, one can then define $\left(\beta, \alpha_{t}\right)$-KMS states $\omega_{\beta}$.

We stress again that it would be premature to envisage these states in analogy to the global equilibrium states in Minkowski spacetime $\mathbf{M}_{\mathbf{0}}$. There is no well-defined thermostatics for $\mathbf{M}$, since many of the concepts involved in the formulation of this theory implicitly make use of the homogeneity of space (additivity of the entropy, for example). In particular, there is no variant of the zeroth law in curved spacetime. Hence, the KMS parameter $\beta$ needs to be interpreted in terms of the local temperature.

For the explicit construction of KMS states for the free scalar field, one needs the following well-known result [HHW67], which is a useful reformulation of the KMS condition of Definition 1.1 .10

Proposition 3.1.2. In the notation of Definition 1.1.10, a state $\omega \in \mathcal{S}(\mathbf{M})$ is $\left(\alpha_{t}, \beta\right)-K M S$ if and only if the Fourier transforms $\hat{F}_{A B}$ and $\hat{G}_{A B}$ of $F_{A B}$ and $G_{A B}$ satisfy

$$
\left.\hat{F}_{A B}\right|_{\mathbb{R}}=\left.e^{\beta(\cdot)} \hat{G}_{A B}\right|_{\mathbb{R}}
$$

in the sense of tempered distributions for all $A, B \in \mathcal{A}(\mathbf{M}) \mathrm{H}^{3}$

Recall the functors which describe the quantum theory of the free scalar field, in particular the lcQFTs $\mathcal{K}$ and $\mathcal{W}$ with state space $\mathcal{S}$, as described in Section 1.2, For the moment, we leave the mass $m^{2}$ and the curvature coupling $\xi$ unspecified.

We begin by reviewing the construction of KMS states for this theory. Assume that $\mathbf{M}$ is stationary and let $\Delta$ be the causal propagator for the free field as defined in equation (1.8). Given the one-parameter-group of time-like isometries $\left\{\chi_{t}\right\}_{t \in \mathbb{R}}$ on $\mathbf{M}$, we define

$$
\Delta_{f, g}(t):=\Delta\left(f, g \circ \chi_{t}^{-1}\right) \text { for } f, g \in \mathcal{D}(M) .
$$

\footnotetext{
${ }^{2}$ If $\mathbf{M}$ is globally hyperbolic, these hypersurfaces are Cauchy surfaces.

${ }^{3}$ More precisely, this has to be understood as first restricting $\hat{G}_{A B}$ to $\mathcal{D}(\mathbb{R})$, then multiplying with $e^{\beta(\cdot)}$, and finally uniquely extending the result to a tempered distribution again.
} 
As a consequence of the CCR, cf. equation 1.10 , the elements $A:=\phi_{\mathrm{M}}\left(f_{1}\right) \ldots \phi_{\mathrm{M}}\left(f_{n}\right)$ and $B:=\phi_{\mathbf{M}}\left(f_{n+1}\right)$ of $\mathcal{A}(\mathbf{M})$ satisfy the algebraic relation

$$
\left[A, \alpha_{t}(B)\right]=\sum_{k=1}^{n} i \Delta_{f_{k}, f_{n+1}}(t) \phi_{\mathbf{M}}\left(f_{1}\right) \ldots \check{\phi}_{\mathbf{M}}\left(f_{k}\right) \ldots \phi_{\mathbf{M}}\left(f_{n}\right),
$$

where indicates that the corresponding field is omitted.

Let $\omega$ be an $\left(\alpha_{t}, \beta\right)$-KMS state on $\mathcal{K}(\mathbf{M})$, then, from equation $(3.1)$, it follows that for the elements $A$ and $B$ one has

$$
\begin{aligned}
\left.\left(1-e^{-\beta(\cdot)}\right) \hat{F}_{A B}\right|_{\mathbb{R}} & =\hat{F}_{A B}\left|\mathbb{R}-\hat{G}_{A B}\right|_{\mathbb{R}} \\
& =\sum_{k=1}^{n} i \hat{\Delta}_{f_{k}, f_{n+1}}(\cdot) \omega^{(n-1)}\left(f_{1}, \ldots, \check{f}_{k}, \ldots, f_{n}\right)
\end{aligned}
$$

with the Fourier transform $\hat{\Delta}_{f_{k}, f_{n+1}}$ of $\Delta_{f_{k}, f_{n+1}}$. Similar arguments for $B$ involving more than one field show that the $n$-point functions of a KMS state can expressed through their $(n-2)$-point function, and thus, ultimately, via its two-point function. This implies that KMS states are quasi-free states, but not necessarily even. If, however, $\omega$ is even, which we assume from now on, $\omega^{(1)}=0$ and one obtains for the two-point function (putting $n=1$ in the definition of $A$ ):

$$
\begin{aligned}
\omega^{(2)}\left(f_{1}, f_{2}\right) & =\frac{i}{\sqrt{2 \pi}} \int_{-\infty}^{\infty} d k \frac{\hat{\Delta}_{f_{1}, f_{2}}(k)}{1-e^{-\beta k}} \\
& =\frac{i}{2 \pi} \int_{-\infty}^{\infty} d k \frac{1}{1-e^{-\beta k}} \int_{-\infty}^{\infty} d t \Delta_{f_{1}, f_{2}}(t) e^{-i k t}
\end{aligned}
$$

which determines $\omega$ completely.

For simplicity, we now specialize to the massless $\left(m^{2}=0\right)$, conformally coupled $\left(\xi=\frac{1}{6}\right)$ scalar field. In Minkowski spacetime $\mathbf{M}_{\mathbf{0}}$, the corresponding causal propagator is given by the formal kernel

$$
\Delta(x, y)=\frac{-1}{2 \pi} \varepsilon\left(x_{0}-y_{0}\right) \delta\left((x-y)^{2}\right),
$$

with $\varepsilon$ denoting the sign function. In the massless case, the Hadamard parametrix is equal to the vacuum two-point function given in equation 1.15 , i.e.

$$
h(x, y)=\lim _{\varepsilon \rightarrow 0} \frac{1}{4 \pi^{2}} \frac{1}{(x-y)^{2}+i \varepsilon\left(x_{0}-y_{0}\right)+\varepsilon^{2}}
$$

One can thus calculate the expectation values for the locally covariant Wick powers in the quasi-free (primary) KMS states $\omega_{\beta}$ with respect to the time evolution $x \mapsto x+t e$ with $e \in V^{+}$The result of the straightforward calculation is, in case of the Wick square,

$$
\omega_{\beta}\left(: \phi_{0}^{2}:(x)\right)=\frac{1}{12 \beta^{2}}
$$

\footnotetext{
${ }^{4}$ Note that in the massive case, the Hadamard parametrix differs from the vacuum two-point function and thus the locally covariant Wick powers differ from Wick powers that are regularised by subtraction of the vacuum. See [SV08] for details.
} 
This means that in any spacetime, $: \phi^{2}$ : can be used as a local thermometer and any state with $\omega\left(: \phi^{2}:(x)\right) \geq 0$ is locally thermal at $x$ with regard to this observable.

In the case of the thermal energy tensor, one has

$$
\omega_{\beta}\left(\epsilon_{0, a b}(x)\right)=\frac{\pi^{2}}{90 \beta^{4}}\left(4 e_{a} e_{b}+\eta_{a b}\right)
$$

cf. [BOR02]. Here, $\eta$ denotes the Minkowski metric $\eta=g_{0}$.

Equations (3.6) and (3.7) fix the thermal functions for the thermal observables we are interested in 5

It is important to note that the thermal energy tensor is traceless in the reference states, which, as was mentioned before, leads to a linear constraint on LTE states. The corresponding equation of state is $E=3 P$, where $E$ is the (thermal) energy density in the rest system $e$ and $P$ is the pressure. Moreover, the temperature dependence of $E$ is given by a Stefan-Boltzmann type law, $E(\beta)=\frac{\pi^{2}}{30 \beta^{4}}$, as expected in a massless theory. This is an example for a non-linear equation of state, in this case between the thermal functions corresponding to : $\phi^{2}$ : and $\epsilon_{00}$.

As we discuss in the next section, the Wick powers of the massless, conformally coupled free field should be conformally covariant. This can be done with a certain choice of renormalization for these fields. We review the concept of locally conformally covariant quantum fields in the sense of Pinamonti [Pin09] here, also correcting a mistake in the original work. In this approach, conformal embeddings between two spacetimes $\mathbf{M}_{1}$ and $\mathbf{M}_{2}$ play an important rôle, i.e. maps $\psi: \mathbf{M}_{1} \rightarrow \mathbf{M}_{2}$ which act like hyperbolic embeddings, cf. Definition 1.1.2, except that they are not isometries. Rather there is a smooth function $\Omega: M_{2} \rightarrow \mathbb{R}$ such that $\psi_{*} g_{1}=\left.\Omega^{-2} g_{2}\right|_{\psi\left(M_{1}\right)}$. Details can be found e.g. in [Wal84, Appendix $\mathrm{D}]$. The weighted action of the conformal embedding $\psi$ with weight $\lambda \in \mathbb{N}$ on test functions is given by the map

$$
\psi_{*}^{(\lambda)}: \mathcal{D}\left(M_{1}\right) \rightarrow \mathcal{D}\left(\psi\left(M_{1}\right)\right), \quad \psi_{*}^{(\lambda)} f(x):=\Omega^{-\lambda}(x) f\left(\psi^{-1}(x)\right) .
$$

Pinamonti generalized the notion of lcQFT to locally conformally covariant QFT by using the category CLoc instead of Loc, which uses conformal embeddings instead of hyperbolic embeddings as morphisms. In other words, a conformally locally covariant QFT is a covariant functor $\mathcal{A}$ between CLoc and Alg. Hence, if $\psi: \mathbf{M}_{1} \rightarrow \mathbf{M}_{2}$ is a conformal

\footnotetext{
${ }^{5}$ In the massive case, the thermal functions look more complicated, but the Wick square is still a monotonous function of $\beta$ and can thus be used as a local thermometer. Details are presented in [Hüb05], see also [SV08].
} 
embedding, the following diagram commutes, and we write $\alpha_{\psi}:=\mathcal{A}(\psi)$ :

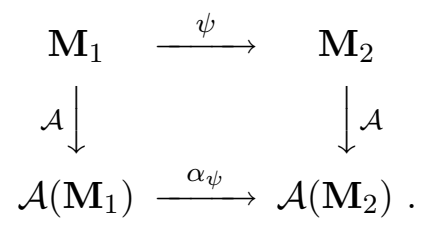

A conformally covariant quantum field of weight $\lambda$ transforms under the action of $\psi$ according to the formula

$$
\alpha_{\psi} \circ \phi_{\mathbf{M}_{1}}^{(\lambda)}=\phi_{\mathbf{M}_{2}}^{(\lambda)} \circ \psi_{*}^{(4-\lambda)}
$$

or, in terms of formal kernels,

$$
\alpha_{\psi} \phi_{\mathbf{M}_{1}}^{(\lambda)}(x)=\Omega^{\lambda}(\psi(x)) \phi_{\mathbf{M}_{2}}^{(\lambda)}(\psi(x))
$$

for $x \in \mathbf{M}_{1}$.

In contrast to lcQFT, this notion of covariance allows one to transplant observables and states from one spacetime to another not only when one is isometrically embedded into the other, but more generally when there is a conformal embedding between them.

Pinamonti has shown that, with a certain choice of renormalization constants, the Wick powers of the massless conformally coupled free scalar field without derivatives are conformally covariant quantum fields. We outline the argument for the case of the Wick square.

Lemma 3.1.3 ([in09]). Consider the locally covariant Wick square $: \phi^{2}:_{\mathrm{M}}=: \phi^{2}:_{h}+\alpha_{0} R_{g}$ for the massless, conformally coupled scalar field. Let $\psi: \mathbf{M}_{1} \rightarrow \mathbf{M}_{2}$ be a conformal embedding, then for $f \in \mathcal{D}\left(M_{1}\right)$

$$
\begin{aligned}
& \alpha_{\psi}: \phi^{2}:_{\mathbf{M}_{1}}(f)-: \phi^{2}:_{\mathbf{M}_{2}}\left(\psi_{*}^{(2)} f\right)=\left(\alpha_{0}-\frac{1}{288 \pi^{2}}\right) . \\
& \cdot \int_{M_{1}}\left(R_{g_{1}}(x)-\Omega^{2}(\psi(x)) R_{\psi_{*} g_{1}}(\psi(x))\right) f(x) \mathrm{d} \mu_{g_{1}}(x) .
\end{aligned}
$$

Therefore, $\mathbf{M} \mapsto: \phi^{2}:_{\mathbf{M}}$ is a conformally covariant quantum field of weight two if and only if the renormalization constant is chosen as $\alpha_{0}=\frac{1}{288 \pi^{2}}$. $^{6}$

Proof. Let $\mathcal{O}_{1}$ be a geodesic normal neighbourhood in $M_{1}$ such that $\psi\left(\mathcal{O}_{1}\right)$ lies inside of a geodesic normal neighbourhood $\mathcal{O}_{2}$ in $M_{2}$. We denote by $h_{1}$ and $h_{2}$ the Hadamard parametrices for the conformally coupled field on $\mathcal{O}_{1}$ and $\mathcal{O}_{2}$ respectively. Consider an

\footnotetext{
${ }^{6}$ Due to two mistakes in the calculations in [Pin09], this result differs from the original. See the proof for an explanation. I am grateful to N. Pinamonti for his support in the identification of these errors.
} 
$f \in \mathcal{D}\left(M_{1}\right)$ with $\left.\operatorname{supp}(f) \subset \mathcal{O}_{1} \cdot\right]^{7}$ Formally, one has

$$
\begin{gathered}
\alpha_{\psi}: \phi^{2}:_{\mathbf{M}_{1}}(f)=\int_{M_{1}}\left(\alpha_{\psi} \phi_{\mathbf{M}_{1}}(x) \alpha_{\psi} \phi_{\mathbf{M}_{1}}(y)-h_{1}(x, y)+c R_{g_{1}}(x)\right) . \\
\cdot f(x) \delta(x, y) \mathrm{d} \mu_{g_{1}}(x) \mathrm{d} \mu_{g_{1}}(y)
\end{gathered}
$$

and

$$
\begin{gathered}
: \phi^{2}:_{\mathbf{M}_{2}}\left(\psi_{*}^{(2)} f\right)=\int_{\psi\left(M_{1}\right)}\left(\phi_{\mathbf{M}_{2}}(\psi(x)) \phi_{\mathbf{M}_{2}}(\psi(y))-h_{2}(\psi(x), \psi(y))+c R_{\psi_{*} g_{1}}(\psi(x))\right) . \\
\cdot \Omega^{-2}(\psi(x)) f(x) \delta(\psi(x), \psi(y)) \mathrm{d} \mu_{\psi_{*} g_{1}}(\psi(x)) \mathrm{d} \mu_{\psi_{*} g_{1}}(\psi(y)) \\
=\int_{M_{1}}\left(\phi_{\mathbf{M}_{2}}(\psi(x)) \phi_{\mathbf{M}_{2}}(\psi(y))-h_{2}(\psi(x), \psi(y))+c R_{\psi_{*} g_{1}}(\psi(x))\right) \\
\cdot \Omega(\psi(x)) \Omega(\psi(y)) f(x) \delta(\psi(x), \psi(y)) \mathrm{d} \mu_{g_{1}}(x) \mathrm{d} \mu_{g_{1}}(y),
\end{gathered}
$$

where we made use of the relation $\mathrm{d} \mu_{\psi_{*} g_{1}}(\psi(x))=\Omega^{4}(\psi(x)) \mathrm{d} \mu_{g_{1}}(x)$.

As the conformally coupled free scalar field $\phi_{M}$ is a conformally covariant quantum field of weight one [Pin09, Prop. 3.2], one obtains

$$
\begin{aligned}
\alpha_{\psi}: \phi^{2}{ }_{\mathbf{M}_{1}}(f)-: \phi^{2}:_{\mathbf{M}_{2}}\left(\psi_{*}^{(2)} f\right) & =\int_{M_{1}}\left(\left[\Omega(\psi(x)) h_{2}(\psi(x), \psi(y)) \Omega(\psi(y))-h_{1}(x, y)\right] \delta(x, y)\right. \\
& \left.+\alpha_{0}\left[R_{g_{1}}(x)-\Omega^{2}(\psi(x)) R_{\psi_{*} g_{1}}(\psi(x))\right]\right) f(x) \mathrm{d} \mu_{g_{1}}(x) \mathrm{d} \mu_{g_{1}}(y) .
\end{aligned}
$$

The coincidence limit of the term involving the Hadamard parametrices has been calculated in [Pin09, Appendix A.2]. Yet there, the Hadamard parametrices are normalized incorrectly; they are in fact one-half of the standard ones which we use here. The thus corrected result reads

$$
\begin{aligned}
\lim _{x \rightarrow y}(\Omega(\psi(x)) & \left.h_{2}(\psi(x), \psi(y)) \Omega(\psi(y))-h_{1}(x, y)\right)= \\
& -\frac{1}{288 \pi^{2}}\left(R_{g_{1}}(x)-\Omega^{2}(\psi(x)) R_{\psi_{*} g_{1}}(\psi(x))\right)
\end{aligned}
$$

and 3.10 follows 8

Pinamonti has also shown that the renormalization constants can be chosen such that the higher Wick powers become conformally covariant. These fields also play an important rôle as thermal observables, which we see in the following.

Let us choose

$$
\mathbf{T}_{2 n}:=\left\{\mathbb{1},: \phi^{2}:, \ldots,: \phi^{2 n}:\right\}
$$

${ }^{7}$ For general $f \in \mathcal{D}\left(M_{1}\right)$ one can use a partition of unity argument in order to reduce the proof to the case at hand.

${ }^{8}$ There is also a sign error in the final result of [Pin09], so that the constant $\alpha_{0}$ is negative while it should be $\alpha_{0}=\frac{1}{288 \pi^{2}}$. This value agrees with the remark in Pag82, below equation (29). 
as the set of thermal observables ${ }^{9}$ In Minkowski spacetime, it holds that

$$
\omega_{0}\left(: \phi^{2 n}:_{0}(x)\right)=(2 n-1) ! ! \omega_{0}\left(: \phi^{2}:_{0}(x)\right)^{n}
$$

when $\omega_{0} \in \mathcal{S}\left(\mathbf{M}_{\mathbf{0}}\right)$ is quasi-free. As was mentioned previously, the reference states $\omega_{\beta}$ at the sharp temperature $\beta$ provide an example of this ${ }^{10}$, so the following simple relation between the corresponding thermal exists:

$$
\omega_{\beta}\left(: \phi^{2 n}:_{0}(0)\right)=(2 n-1) ! ! \omega_{\beta}\left(: \phi^{2}:_{0}(0)\right)^{n}=\frac{(2 n-1) ! !}{12^{n} \beta^{2 n}} .
$$

The following observation is a direct consequence of this fact.

Observation 3.1.4. A quasi-free state in Minkowski spacetime is $\mathbf{T}_{2 n}(x)$-thermal if and only if it is $\mathbf{T}_{2}(x)$-thermal. As a consequence, a $\mathbf{T}_{2}(x)$-thermal quasi-free state has a sharp local temperature.

The first statement indicates that usage of the larger space $\mathbf{T}_{2 n}$ instead of $\mathbf{T}_{2}$ does not provide any additional thermal information in quasi-free states. However, for general states, $\mathbf{T}_{2 n}$-thermality represents a much stronger constraint than $\mathbf{T}_{2}$-thermality. The second item in the observation can be justified as follows: a quasi-free $\mathbf{T}_{2}(x)$-thermal state $\omega_{0}$ is also $\mathbf{T}_{4}(x)$-thermal and thus satisfies $\omega_{0}\left(: \phi^{4}:_{0}(x)\right)=\omega_{\rho_{x}}\left(: \phi^{4}:_{0}(0)\right)$. But by (3.12) this already implies

$$
\int \frac{d \rho_{x}(\beta)}{\left(\beta^{2}\right)^{2}}=\left(\int \frac{d \rho_{x}(\beta)}{\beta^{2}}\right)^{2}
$$

so $\rho_{x}$ must be a Dirac measure.

The validity of these conclusions rests on relation (3.12), which so far is only valid in Minkowski spacetime. In the more general case of a curved spacetime $\mathbf{M}$, we need to take into account the renormalization freedom, as we explain in the following. Let $\omega \in \mathcal{S}(\mathbf{M})$ be a state for the massless, conformally coupled scalar field. If $\omega$ is quasi-free, the Hadamard normal ordered Wick powers with odd exponent vanish and, moreover, the ones with even exponent enjoy a property which corresponds to 3.12 :

$$
\omega\left(: \phi^{2 n}:{ }_{h}(x)\right)=(2 n-1) ! ! \omega\left(: \phi^{2}:{ }_{h}(x)\right)^{n}
$$

However, according to the results by Hollands and Wald [HW01] on the ambiguity in the definition of the full locally covariant Wick powers, see equation 1.31, we have

$$
\omega\left(: \phi^{2 n}:(x)\right)=\sum_{k=0}^{2 n}\left(\begin{array}{c}
2 n \\
k
\end{array}\right) C_{2 n-k}(x) \omega\left(: \phi^{k}: h(x)\right),
$$

\footnotetext{
${ }^{9}$ Note that all odd Wick powers vanish in the reference states. Hence these fields do not represent suitable thermal observables.

${ }^{10}$ General reference states are not quasi-free, as mixtures of quasi-free states need not be quasi-free.
} 
where $C_{0}(x):=1{ }^{11}$ Therefore, the analogue of relation $(3.12)$ is in general more complicated and Observation 3.1 .4 fails to hold in curved spacetime. In particular one finds that quasi-free states generally do not have a sharp temperature. However, one may impose relation 3.13 for the full locally covariant Wick powers up to order $2 n$, in which case the renormalization freedom is greatly reduced. Namely, we have the following result.

Proposition 3.1.5. Let $n \in \mathbb{N}$. If $\omega \in \mathcal{S}(\mathbf{M})$ is quasi-free, then

$$
\omega\left(: \phi^{2 n}:(x)\right)=(2 n-1) ! ! \omega\left(: \phi^{2}:(x)\right)^{n}
$$

if and only if

$$
C_{2 m}=(2 m-1) ! ! C_{2}{ }^{m} \quad \text { for all } m=1,2, \ldots, n \text {. }
$$

Proof. Using that in quasi-free states Hadamard normal ordered Wick powers with odd exponents vanish, we compute

$$
\begin{aligned}
\omega\left(: \phi^{2 n}:(x)\right) & =\sum_{k=0}^{n}\left(\begin{array}{l}
2 n \\
2 k
\end{array}\right) C_{2(n-k)}(x) \omega\left(: \phi^{2 k}: h(x)\right) \\
& \stackrel{3.13}{=} \sum_{k=0}^{n}\left(\begin{array}{l}
2 n \\
2 k
\end{array}\right) C_{2(n-k)}(x)(2 k-1) ! ! \omega\left(: \phi^{2}: h(x)\right)^{k} \\
& =\sum_{k=0}^{n}\left(\begin{array}{l}
2 n \\
2 k
\end{array}\right) C_{2(n-k)}(x)(2 k-1) ! !\left(\omega\left(: \phi^{2}:(x)\right)-C_{2}(x)\right)^{k} \\
& =\sum_{k=0}^{n} \sum_{l=0}^{k} \underbrace{(-1)^{k-l}\left(\begin{array}{c}
2 n \\
2 k
\end{array}\right)\left(\begin{array}{c}
k \\
l
\end{array}\right)(2 k-1) ! !}_{:=M_{k l}^{n}} C_{2(n-k)}(x) C_{2}(x)^{k-l} \omega\left(: \phi^{2}:(x)\right)^{l},
\end{aligned}
$$

where in the last step we used the appropriate binomial formula. To simplify, we order the terms in the sum by powers of $\omega\left(: \phi^{2}:(x)\right)$ :

$$
\omega\left(: \phi^{2 n}:(x)\right)=\sum_{l=0}^{n}\left(\sum_{k=l}^{n} M_{k l}^{n} C_{2(n-k)}(x) C_{2}(x)^{k-l}\right) \omega\left(: \phi^{2}:(x)\right)^{l} .
$$

Therefore, equation 3.15 applies if and only if

$$
\sum_{l=0}^{n}\left(\sum_{k=l}^{n} M_{k l}^{n} C_{2(n-k)}(x) C_{2}(x)^{k-l}\right) \omega\left(: \phi^{2}:(x)\right)^{l}=(2 n-1) ! ! \omega\left(: \phi^{2}:(x)\right)^{n}
$$

for all quasi-free states $\omega \in \mathcal{S}(\mathbf{M})$. We compare coefficients on both sides and find

$$
\sum_{k=l}^{n} M_{k l}^{n} C_{2(n-k)}(x) C_{2}(x)^{k-l}=0 \quad \text { for } l=0,1, \ldots, n-1
$$

\footnotetext{
${ }^{11}$ The $C_{i}(x)$ constitute the renormalization freedom (recall that $C_{2}=\alpha_{0} R$ with $\alpha_{0} \in \mathbb{R}$, for example).
} 
(the coefficients corresponding to the $n$-th power yield no constraints on the $C_{i}$ ).

We now argue by induction. Using the relation for $l=n-2$, it is easy to check from (3.19) that our claim is true for $C_{4}$, i.e. $C_{4}(x)=3 C_{2}(x)^{2}$. Assume now that for some $m<n$ it holds that $C_{2 k}=(2 k-1) ! ! C_{2}{ }^{k}$ for all $k<m$. We need to show that in this case $C_{2 m}=(2 m-1) ! ! C_{2}{ }^{m}$ also.

For this, we use $(3.19)$ with $l=n-m$ and find

$$
\sum_{k=n-m}^{n} \frac{(-1)^{k-(n-m)}}{2^{k-(n-m)}} \frac{C_{2(n-k)} C_{2}^{k-(n-m)}}{(2(n-k)) !(k-(n-m)) !}=0 .
$$

Applying our induction assumption leads to

$$
C_{2 m}=-\frac{(2 m) !}{2^{m}}\left[\sum_{k=1}^{m} \frac{(-1)^{k}}{(m-k) ! k !}\right] C_{2}^{m} .
$$

But the sum in square brackets is simply

$$
\sum_{k=1}^{m} \frac{(-1)^{k}}{(m-k) ! k !}=-\frac{1}{m !}+\sum_{k=0}^{m} \frac{(-1)^{k} 1^{m-k}}{(m-k) ! k !}=-\frac{1}{m !}
$$

and we thus find

$$
C_{2 m}=\frac{(2 m) !}{2^{m} m !} C_{2}^{m}=(2 m-1) ! ! C_{2}{ }^{m},
$$

which proves our claim.

Note that condition 3.16 is non-trivial. For example $C_{4}$ is of the form

$$
C_{4}=\gamma_{0} R^{2}+\gamma_{1} \square R+\gamma_{2} R_{a b} R^{a b}+\gamma_{3} R_{a b c d} R^{a b c d}
$$

with real constants $\gamma_{i}$. Remembering that $C_{2}=\alpha_{0} R$, the proposition states that if 3.15 is to hold, then $\gamma_{1}=\gamma_{2}=\gamma_{3}=0$ and $\gamma_{0}=3 \alpha_{0}^{2}$. More generally,

$$
C_{2 m}=(2 m-1) ! ! \alpha_{0}^{m} R^{m}
$$

for all $m=1,2, \ldots, n$. We therefore conclude that, once we have fixed the Wick square, condition 3.15) completely fixes the renormalization ambiguity for the even Wick powers up to order $2 n$. Choosing $\alpha_{0}=\frac{1}{288 \pi^{2}}$, the Wick square is a conformally covariant quantum field. If we also adopt condition (3.15), all even Wick powers are also fixed. One can employ the methods used in the proof of [Pin09, Theorem 3.1] to show that these fields are also conformally covariant for this choice.

Corollary 3.1.6. If $C_{2 m}=(2 m-1) ! ! \alpha_{0}^{m} R^{m}$ for all $m=1,2, \ldots, n$, then the following statement is true: A quasi-free state $\omega \in \mathcal{S}(\mathbf{M})$ is $\mathbf{T}_{2 n}(x)$-thermal if and only if it is $\mathbf{T}_{2}(x)$-thermal. If $n \geq 2$, then the state has a sharp local temperature. 
Therefore, if condition (3.15) holds, the combinatorics for quasi-free $\mathbf{T}_{2 n}(x)$-thermal states in curved spacetime is the same as in Minkowski spacetime. In this regard, it is interesting to note that all of the examples considered in the next section are quasi-free, so if they are $\mathbf{T}_{2}$-thermal, they are also $\mathbf{T}_{2 n}$-thermal and must have a sharp temperature. This is of importance, because if the local temperature, as measured by $\tilde{\epsilon}_{00}$, for example (see below), deviates from the local temperature as measured by : $\phi^{2}$ : (we refer to a non-linear equation of state, namely the Stefan Boltzmann law), we cannot have local thermality because no sharp temperature is possible. Finally, we would like to point out that Proposition 3.1.5 can be generalized to the massive case straightforwardly.

We now wish to discuss the thermal energy tensor, i.e. we consider the set of thermal observables

$$
\mathbf{T}^{(2)}:=\left\{\mathbb{1},: \phi^{2}:, \epsilon_{a b}\right\},
$$

which was already introduced in equation 2.15 , we now proceed to discuss $\mathbf{T}^{(2)}$-thermality in curved spacetime. Recall that

$$
\epsilon_{a b}:=-: \phi\left(\nabla_{a} \nabla_{b} \phi\right):+\frac{1}{4} \nabla_{a} \nabla_{b}: \phi^{2}:
$$

In a theory with curvature coupling $\xi=1 / 6$ and mass $m^{2}$, in Minkowski spacetime $\mathbf{M}_{0}$, one finds

$$
T_{0, a b}=\left\{\epsilon_{0, a b}-\frac{1}{4} g_{a b}: \phi P \phi:\right\}+\frac{1}{12}\left(\partial_{a} \partial_{b}-\eta_{a b} \square\right): \phi^{2}:_{0}-\frac{1}{4} g_{a b}: \phi P \phi: .
$$

Note that the terms $-\frac{1}{4} g_{a b}: \phi P \phi$ : do not appear in the standard normal ordering procedure for the Wick powers in $\mathbf{M}_{0}$ (which is employed in [BOR02]). However, as we mentioned earlier, for the Hadamard normal ordered Wick powers, these terms generally do not vanish and one has to take them into account, see [SV08] for a discussion. In the case of the conformally coupled massless field, however, both normal ordering prescriptions coincide and : $\phi P \phi$ : vanishes in $\mathbf{M}_{0}$.

In 3.22 , we have written $T_{0, a b}$ such that the term in curly brackets has trace $\left(-\frac{1}{4} \square+\right.$ $\left.m^{2}\right): \phi^{2}:_{0}$ (just like the thermal energy tensor when renormalized as in [BOR02]), the second term has trace $\frac{1}{4} \square: \phi^{2}:_{0}$ and the last term is responsible for the anomaly (which vanishes in the massless case in Minkowski spacetime). Equation (3.22) shows that, for non-homogeneous LTE states in $\mathbf{M}_{0}$, the expectation value of the full energy momentum generally does not coincide with the thermal energy, because additional contributions appear due to transport terms which are not present in $\epsilon_{a b}$ [BOR02.

In curved spacetime, $\epsilon_{a b}$ is related to the energy momentum tensor $T_{a b}$ by

$$
T_{a b}=\left\{\epsilon_{a b}+\frac{1}{6} R_{a b}: \phi^{2}:-\frac{1}{4} g_{a b}: \phi P \phi:\right\}+\frac{1}{12}\left(\nabla_{a} \nabla_{b}-g_{a b} \square\right): \phi^{2}:-\frac{1}{4} g_{a b}: \phi P \phi:,
$$


which follows from the definition of $T_{a b}$, cf. Section 1.2.3 by application of the Leibniz rule. The term in curly brackets includes the thermal observables $\epsilon_{a b},: \phi^{2}$ : and $\mathbb{1}$, while the next one, the transport term, is made up of non-thermal observables plus a contribution that, in a valid renormalization scheme where $\nabla^{a} T_{a b}=0$, is responsible for the anomaly. The split of $T_{a b}$ into a thermal and a non-thermal part suggests that we use

$$
\tilde{\epsilon}_{a b}:=\epsilon_{a b}+\frac{1}{6} R_{a b}: \phi^{2}:-\frac{1}{4} g_{a b}: \phi P \phi:
$$

as a new definition of the thermal energy tensor.12 Accordingly, we define

$$
\tilde{\mathbf{T}}^{(2)}:=\left\{\mathbb{1},: \phi^{2}:, \tilde{\epsilon}_{a b}\right\}
$$

as a new set of thermal observables. In Section 3.2 , we test KMS states in simple stationary curved spacetimes for both, $\tilde{\mathbf{T}}^{(2)}$ - and $\mathbf{T}^{(2)}$-thermality, in order to decide which notion of thermality is physically justified. It is important to emphasize that $\tilde{\mathbf{T}}^{(2)}$ and $\mathbf{T}^{(2)}$ coincide in Minkowski spacetime $\mathbf{M}_{0}$ and therefore give rise to the same thermal functions 3.6 . and 3.7 .

In both cases, thermal energy in LTE states in curved spacetime differs generally from their full energy content. This is in conflict with the usual practise in cosmology, where thermal energy is identified with the full energy momentum. However, as was already pointed out in [Buc03], in view of the discussion above, this identification can only be an approximation, because of the additional "non-thermal" contributions due to transport terms in the energy momentum tensor described before.

We can now discuss the influence of the thermal energy tensor on the dynamics of the temperature. We start by computing the trace of $\epsilon_{a b}$, which yields

$$
\epsilon_{a}^{a}=-\left(-\frac{1}{4} \square+\frac{1}{6} R+m^{2}\right): \phi^{2}:+: \phi P \phi:
$$

This, by (3.24), implies that

$$
\tilde{\epsilon}_{a}^{a}=-\left(-\frac{1}{4} \square+m^{2}\right): \phi^{2}:
$$

For a more detailed understanding of $\epsilon^{a}{ }_{a}$, it is therefore necessary to know the explicit form of the anomaly : $\phi P \phi$ :. As follows from the discussion at the end of section 1.2.3, in a prescription where the quantum energy momentum tensor is divergence-free, one has

$$
: \phi P \phi:=-\left(\frac{1}{4 \pi^{2}}\left[v_{1}\right]+m^{2}\left(\alpha_{0} R+\alpha_{1} m^{2}\right)+g^{a b} T_{a b}^{R F}\right) \mathbb{1} .
$$

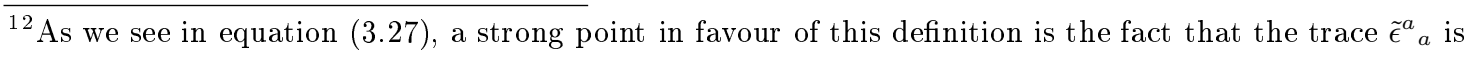
of the same form as in Minkowski spacetime.
} 
Using equation (1.39), we also find that

$$
g^{a b} T_{a b}^{R F}=\left(6 \beta_{1}+2 \beta_{2}\right) \square R-\beta_{3} m^{2} R+4 \beta_{4} m^{4} .
$$

Again, we specialize to the massless $\left(m^{2}=0\right)$ field for simplicity. In this case, the coincidence limit of the first Hadamard coefficient is given by [DF06]:13

$$
\left[v_{1}\right]=\frac{1}{720}\left(\square R+R_{\rho \sigma \tau \kappa} R^{\rho \sigma \tau \kappa}-R_{\rho \sigma} R^{\rho \sigma}\right) .
$$

Alternatively, in terms of the Weyl tensor $C_{\rho \sigma \tau \kappa}: 14$

$$
\left[v_{1}\right]=\frac{1}{720}\left(\square R+C_{\rho \sigma \tau \kappa} C^{\rho \sigma \tau \kappa}+R_{\rho \sigma} R^{\rho \sigma}-\frac{1}{3} R^{2}\right) .
$$

Hence

$$
\begin{aligned}
\epsilon_{a}^{a} & =-\left(-\frac{1}{4} \square+\frac{1}{6} R\right): \phi^{2}: \\
& -\left\{\frac{1}{2880 \pi^{2}}\left(\square R+C_{\rho \sigma \tau \kappa} C^{\rho \sigma \tau \kappa}+R_{\rho \sigma} R^{\rho \sigma}-\frac{1}{3} R^{2}\right)+\left(6 \beta_{1}+2 \beta_{2}\right) \square R\right\} \mathbb{1} .
\end{aligned}
$$

Assume now that $\omega \in \mathcal{S}(\mathbf{M})$ is $\mathbf{T}^{(2)}$-thermal in some open region $\mathcal{O} \subset M$. Since the thermal energy tensor is trace-less in the reference states and $\omega\left(: \phi^{2}(x):\right)=\frac{1}{12} \overline{T^{2}}(x)$ for the local temperature distribution $x \mapsto \overline{T^{2}}(x)$, we find that

$$
\begin{array}{r}
\left(-\frac{1}{4} \square+\frac{1}{6} R\right) \frac{\overline{T^{2}}(x)}{12}=-\frac{1}{2880 \pi^{2}}\left(\square R+C_{\rho \sigma \tau \kappa} C^{\rho \sigma \tau \kappa}+R_{\rho \sigma} R^{\rho \sigma}-\frac{1}{3} R^{2}\right) \mathbb{1} \\
-\left(6 \beta_{1}+2 \beta_{2}\right) \square R \mathbb{1},
\end{array}
$$

for all $x \in \mathcal{O}$, a relation which we already mentioned in Section 2.2.2. Note that not only does the renormalization of the Wick square enter in this equation, but also the constants $\beta_{1}, \beta_{2}$ which appear as renormalization freedom for the energy momentum tensor.

On the one hand, equation 3.30 describes the dynamics of the local temperature in a $\mathbf{T}^{(2)}$-thermal state in curved spacetime and represents a generalization of the corresponding equation in Minkowski spacetime [Buc03]. On the other hand, it is a necessary condition for a state to be $\mathbf{T}^{(2)}$-thermal which can be checked in examples without the need to compute all of the components of the thermal energy tensor. It is useful to rewrite 3.30 in a way that also exposes its dependence on $\alpha_{0}$, the renormalization of the Wick square:

$$
\begin{aligned}
\left(-\frac{1}{4} \square+\frac{1}{6} R\right) \omega\left(: \phi^{2}:_{h}(x)\right)=- & \frac{1}{2880 \pi^{2}}\left(\square R+C_{\rho \sigma \tau \kappa} C^{\rho \sigma \tau \kappa}+R_{\rho \sigma} R^{\rho \sigma}-\frac{1}{3} R^{2}\right) \mathbb{1} \\
& -\left(\left(6 \beta_{1}+2 \beta_{2}-\frac{\alpha_{0}}{4}\right) \square R+\frac{\alpha_{0}}{6} R^{2}\right) \mathbb{1} .
\end{aligned}
$$

\footnotetext{
${ }^{13}$ See also [Hac10] for a clear explanation on how to compute such quantities.

${ }^{14}$ This form is sometimes more convenient, because the Weyl tensor vanishes in conformally flat spacetimes, see [Wal84].
} 
In contrast - using the modified version $\tilde{\epsilon}_{a b}$ of the thermal energy tensor - $\tilde{\mathbf{T}}^{(2)}$-thermality implies the following evolution equation for the temperature:

$$
\square \omega\left(: \phi^{2}:(x)\right)=0, \quad \text { i.e. } \square \omega\left(: \phi^{2}:_{h}(x)\right)=-\alpha_{0} \square R,
$$

which is much simpler and has exactly the same form as in Minkowski spacetime.

We note that neither $\epsilon_{a b}$ nor $\tilde{\epsilon}_{a b}$ is divergence-free in a general curved spacetime (in contrast to Minkowski spacetime). In a static spacetime, by Gauss' law this implies that, while the total energy is conserved, the local thermal energy is not. This means that in LTE states there may be an influx or a loss of thermal energy even in passive states like the KMS states we discuss in the next section.

Using these KMS states, we show that $\tilde{\epsilon}_{a b}$ is a more sensible thermal observable than $\epsilon_{a b}$ when it comes to measuring the thermal energy. It is worth stressing that in cosmology the local temperature is determined by measuring Planck's formula, which is then ad hoc identified with the expectation value of the 00-component of the full energy momentum tensor in a co-moving cosmic frame. Our discussion indicates that the correct choice would be to use $\tilde{\epsilon}_{00}$ instead.

\subsection{Local Thermal Equilibrium and KMS States in Stationary Spacetimes}

In this section, we investigate the LTE properties of various KMS states in simple stationary spacetimes. The latter are treated in many textbooks, e.g. [BD84, Wal84, HE73, MTW73], which is why we are very brief about the geometry of these well-known examples.

The motivation for this discussion stems from a question we have alluded to before: how thermal are KMS states in curved spacetime with regard to our definition of local equilibrium? It is important to realize that, a priori, there is no reason to expect these states to be in LTE, apart from the fact that they are passive with respect to their corresponding dynamics. The observers following these dynamics, however, are in general accelerated and their trajectories can be quite complicated, which makes it hard to draw a direct analogy to the global equilibrium states in Minkowski spacetime. Even as, for a certain set of observers, curvature and acceleration seem to conspire to make the state passive, the rôle of the KMS parameter $\beta$ as a temperature is not clear at all. Therefore, the definition of LTE by means of gauging thermal observables in Minkowski spacetime and using them to probe thermality seems a good way to discern the features which the "curved spacetime KMS states" have in common with the global equilibrium states in Minkowski spacetime. 
We review previous results for the Wick square in Rindler, Einstein static, Static de Sitter, Anti de Sitter, spatially flat Robertson-Walker and Schwarzschild spacetimes. We also add our own result for wedge dynamics in Anti de Sitter spacetime. All of these findings serve to underline the fact, already observed in [BS07], that for the KMS states in these examples the KMS parameter $\beta$ generally differs from the inverse local temperature. Sometimes, these states even fail to be in LTE, depending on the values of $\beta$ and the spacetime point - even for a simple thermal observable such as the Wick square.

We also test the thermality of these states using the thermal energy tensor. As we have explained in Section 3.1, this observable puts a linear constraint on LTE states, see equations (3.31) and 3.32). In the examples, this leads to the conclusion that only a small number of KMS states fulfil such constraints, which shows that in most cases the curved spacetime KMS states have a rather small degree of thermality. Again, this should not come as a surprise, since thermality with respect to the thermal energy tensor is a strong constraint - the resulting condition is not an inequality as in the case of the Wick square, but a sharp equality. Moreover, the thermal energy tensor contains derivatives and is therefore sensitive to curvature effects which modify the original equations of state. The examples lead us to adopt the new thermal energy tensor 3.24 as the correct observable for measuring the thermal energy.

When testing thermal observables in examples, another important problem must be faced, namely the choice of renormalization of the Wick square and the thermal energy tensor. This amounts to a fixing of the constants $\alpha_{0}, \beta_{1}$ and $\beta_{2}$ as discussed in Section 3.1. We propose fixing $\alpha_{0}$ such that the Wick square is a conformally covariant quantum field, which differs from the choice made in [BS07].

The first examples are given by Rindler, static de Sitter and Anti de Sitter spacetime, which are (subsets of) maximally symmetric spaces ${ }^{15}$ On such spacetimes, for each geodesic there is a Killing vector field $K$ for which the geodesic is a flow line 16 In contrast, not every flow line of $K$ is a geodesic, and the non-geodesic flow line of $K$ describe accelerated motion with acceleration given by

$$
A=\frac{\nabla_{K} K}{-g(K, K)}
$$

For future reference, we denote by $a^{2}:=-g(A, A)$ the square of the four accelerations along the flow lines of $K$. If $K$ is time-like Killing, the quantity $-g(K, K)$ is positive and constant along the flow lines of $K$. Moreover, if $g(K, K)=-1$ along the curve, then the flow parameter is the proper time of an observer following this trajectory.

\footnotetext{
${ }^{15}$ In four dimensions, this means that there are ten independent Killing vector fields in these spacetimes.

${ }^{16}$ See [NPT96] for this and for the following claims.
} 
Rindler spacetime We start with Rindler spacetime $\mathbf{R}$. This flat spacetime can be realized as the subset $\left\{x \in \mathbb{R}^{4}\left|x^{1}>\right| x^{0} \mid\right\}$ of Minkowski spacetime, the metric $g$ being the Minkowski metric, restricted to this subset. There is a positive time-like Killing vector $K=x_{1} \partial_{0}-x_{0} \partial_{1}$ which generates the Lorentz boosts in $x^{1}$-direction. Petersen [Pet07] has investigated the corresponding KMS states $\omega_{\beta}$. His result for the Wick square reads:

$$
\omega_{\beta}\left(: \phi^{2}:(x)\right)=\frac{1}{-g_{x}(K, K)}\left(\frac{1}{12 \beta^{2}}-\frac{1}{12(2 \pi)^{2}}\right) .
$$

Note that this is equal to the expectation value of the Hadamard normal ordered Wick square, because the renormalization freedom is not present due to the flatness of Rindler spacetime. Note also that

$$
\frac{1}{-g_{x}(K, K)}=a^{2}(x)
$$

where $a^{2}(x)$ is the square of the four acceleration of the trajectory passing through $x . \omega_{2 \pi}$ is the Unruh state (i.e. the restriction of the Minkowski vacuum), which has $\omega_{2 \pi}\left(: \phi^{2}:\right)=0$, i.e. a local temperature of zero. KMS-States with $\beta>2 \pi$ are not in local equilibrium, because in that case $\omega_{\beta}\left(: \phi^{2}:\right)<0$.

Applying the linear constraint arising from $\epsilon_{a b}$ (3.30) or $\tilde{\epsilon}_{a b} 3.32$ to 3.34 immediately yields the constraint $\beta \stackrel{!}{=} 2 \pi$ in both cases, which shows that of all the $\beta$-KMS states with respect to the dynamics of an uniformly accelerated observer in Minkowski spacetime, only the Unruh state can be in LTE with regard to the thermal energy tensor. Already in this example we witness the restrictive nature of the linear constraint which arises from the trace of the thermal energy tensor.

de Sitter spacetime The next example is de Sitter spacetime $\mathbf{d} \mathbf{S}_{\alpha}$, which is maximally symmetric with constant positive curvature. $\mathbf{d S}_{\alpha}$ can be represented as hyperboloid, embedded in $\mathbb{R}^{5}$,

$$
\mathrm{dS}_{\alpha}:=\left\{x \in \mathbb{R}^{5} \mid-x_{0}^{2}+x_{1}^{2}+x_{2}^{2}+x_{3}^{2}+x_{4}^{2}=\alpha^{2}\right\}
$$

where $\alpha \in \mathbb{R} \backslash\{0\}$ is the radius of $\mathrm{dS}_{\alpha}$. The metric $g$ is given by the Minkowski metric of ambient $\mathbb{R}^{5}$, restricted to $d S_{\alpha}$.

In de Sitter spacetime, there are no time-like vector fields which are also geodesic. Moreover, a freely falling observer following a geodesic world line $\gamma$ does not have a global notion of dynamics, because the corresponding Killing vector field is not time-like everywhere and hence the union of the past and the future of $\gamma$ is not all of $\mathbf{d S}_{\alpha}$. By symmetry ${ }^{17}$, we can focus attention to the Killing vector field which can be written as

\footnotetext{
${ }^{17}$ The symmetry group of $\mathbf{d} \mathbf{S}_{\alpha}$ is the restriction of the proper orthochronous Lorentz group of ambient $\mathbb{R}^{5}$ to $d S_{\alpha}$.
} 
$K_{1}:=x_{1} \partial_{0}-x_{0} \partial_{1} . K_{1}$ is positive time-like only in the wedge

$$
W^{+}=\left\{x \in d S_{\alpha}|| x_{0} \mid<x_{1}\right\}
$$

The Killing flow generated by $K_{1}$ can be represented as

$$
\chi: t \mapsto\left(\begin{array}{ccc}
\cosh (t) & \sinh (t) & \\
\sinh (t) & \cosh (t) & \\
& & \mathbb{1}_{3}
\end{array}\right) .
$$

$W_{1}^{+}$is the causal completion of the geodesic $\gamma_{0}(t):=\chi_{t}(y)$. Here, $y:=(0, \alpha, 0,0,0)$ and $t$ is the Killing flow parameter, which is related to the proper time $s$, as measured by an observer following $\gamma_{0}$, by $s=\alpha t$. We note that $W_{1}^{+}$is also the causal completion of any other time-like curve contained in it and moreover it is bounded by a bifurcate event horizon at $x_{0}=x_{1}$. As a globally hyperbolic spacetime, the interior of the wedge is called static de Sitter spacetime, which we denote by $\mathbf{d} \mathbf{S}_{\alpha \text {,stat }}$.

We denote by $\left\{\alpha_{t}\right\}_{t \in \mathbb{R}}$ the one-parameter group of automorphisms induced by $\left\{\chi_{t}\right\}_{t \in \mathbb{R}}$, acting on the extended algebra of observables $\mathcal{W}\left(\mathbf{d} \mathbf{S}_{\alpha, \text { stat }}\right)$. For $\beta>0$, one may construct $\left(\beta, \alpha_{t}\right)$-KMS states $\omega_{\beta}$, and Buchholz and Schlemmer [BS07, Sch05] have calculated the expectation value of the Wick square in these states: ${ }^{18}$

$$
\omega_{\beta}\left(: \phi^{2}:(x)\right)=\frac{1}{-g_{x}\left(K_{1}, K_{1}\right)}\left(\frac{1}{12 \beta^{2}}-\frac{1}{12(2 \pi)^{2}}\right)-\frac{1}{24 \pi^{2} \alpha^{2}}+\alpha_{0} \frac{12}{\alpha^{2}} .
$$

Note that apart from the special case $\beta=2 \pi$, the Gibbons-Hawking state, the $\omega_{\beta}$ cannot be extended to Hadamard states on $\mathcal{W}\left(\mathbf{d} \mathbf{S}_{\alpha}\right)$ [NPT96].

The quantity $-g_{x}\left(K_{1}, K_{1}\right)>0$, being constant along the flow lines of $K_{1}$, is a characteristic of the Killing trajectory through $x \in W^{+}$. It is related to the acceleration $a^{2}(x):=g_{x}(A, A)$, cf. equation 3.33 , by

$$
\frac{1}{-g_{x}\left(K_{1}, K_{1}\right)}=a^{2}(x)+\frac{1}{\alpha^{2}}
$$

\footnotetext{
${ }^{18}$ In fact, there has been some confusion regarding this result as presented in [BS07]. On page 5, as Stottmeister [Sto09, p. 64] has pointed out, there is an error in the expansion of the Hadamard parametrix (it should read $\cdots+\frac{R}{192 \pi^{2}}$ instead of the value given there), which then reappears in the equations on page 6 (namely in the calculation preceding eqn. (11)). However, Schlemmer calculated the expectation values of the Hadamard normal ordered Wick square correctly in his Diploma thesis [Sch05 p. 53], on which [BS07, eqn. (11)] and the value of $\alpha_{0}=\frac{1}{192 \pi^{2}}$ given there are based. Thus the errors mentioned here merely occur in the presentation of the steps leading to these final results, which are indeed correct. Unfortunately, Stottmeister took these mistakes into account when he gave a "corrected" constant $\alpha_{0}$ and used it in the interpretation of his results for Einstein Static and Anti de Sitter Spacetime. Even though he computed the expectation values of the Hadamard normal ordered Wick square correctly in these examples, his conclusions therefore need to be re-examined. I am grateful for the support of the authors in the identification of these errors.
} 
Note that an observer following the Killing trajectory through $x \in W_{1}^{+}$has proper time $s(x)=\sqrt{-g_{x}\left(K_{1}, K_{1}\right)} t$, where $t$ is the Killing flow parameter of $K_{1}$. The dynamics of these observers is implemented by the one parameter group of automorphisms $\left\{\alpha_{s(x)}\right\}_{t}$ and it is easy to see that the $\omega_{\beta}$ are KMS states with respect to this one parameter group of automorphisms, albeit with new parameter

$$
\tilde{\beta}(x):=\frac{\beta}{\sqrt{-g_{x}\left(K_{1}, K_{1}\right)}} .
$$

Therefore, these observers feel a shift in the KMS parameter of these states; sometimes called the Tolman redshift.

Using $R=12 / \alpha^{2}, R_{\rho \sigma} R^{\rho \sigma}-\frac{1}{3} R^{2}=-12 / \alpha^{4}$ and the fact that $\mathbf{d} \mathbf{S}_{\alpha, \text { stat }}$ is conformally flat (i.e. the Weyl tensor vanishes), we find the following constraint resulting from the dynamics of the temperature, 3.31:

$$
\frac{\alpha^{2}}{-g_{x}\left(K_{1}, K_{1}\right)}\left(\frac{1}{-g_{x}\left(K_{1}, K_{1}\right)}+\frac{1}{2 \alpha^{2}}\right)\left(\frac{1}{12 \beta^{2}}-\frac{1}{12(2 \pi)^{2}}\right)-\frac{1}{12 \pi^{2} \alpha^{2}}=\frac{1}{240 \pi^{2} \alpha^{2}}-\frac{24}{\alpha^{2}} \alpha_{0} .
$$

This equation seems quite restrictive, but if we use $\tilde{\epsilon}_{a b}$ instead, we obtain the condition

$$
\left(\frac{1}{-g_{x}\left(K_{1}, K_{1}\right)}+\frac{1}{2 \alpha^{2}}\right)\left(\frac{1}{12 \beta^{2}}-\frac{1}{12(2 \pi)^{2}}\right)=0,
$$

which shows that the Gibbons Hawking state is the only KMS state which is in LTE with respect to this thermal observable, because the first factor is always positive in $\mathbf{d} \mathbf{S}_{\alpha, \text { stat }}$. Recall that in Rindler spacetime, we have the same result for the Unruh state. This leads us to adopt $\tilde{\epsilon}_{a b}$ as the correct choice for the thermal energy tensor.

Anti de Sitter spacetime The next example is Anti de Sitter spacetime. Proper Anti de Sitter spacetime $\mathbf{A d} \mathbf{S}_{\alpha}^{\prime}$ is the maximally symmetric spacetime with constant negative curvature. As de Sitter spacetime, $\mathbf{A d S}_{\alpha}^{\prime}$ is realized as a hyperquadric in $\mathbb{R}^{5}$ :

$$
\operatorname{AdS}_{\alpha}^{\prime}:=\left\{x \in \mathbb{R}^{5} \mid-\left(x^{0}\right)^{2}-\left(x^{1}\right)^{2}+\left(x^{2}\right)^{2}+\left(x^{3}\right)^{2}+\left(x^{4}\right)^{2}=-\alpha^{2}\right\},
$$

where $\alpha \in \mathbb{R} \backslash\{0\}$ again denotes the radius. The metric $g$ is the Minkowski metric of ambient $\mathbb{R}^{5}$, restricted to $\operatorname{AdS}^{\prime}{ }_{\alpha}$. However, unlike the other maximally symmetric spacetimes $\mathbf{M}_{\mathbf{0}}$ and $\mathbf{d} \mathbf{S}_{\alpha}$, proper Anti de Sitter spacetime $\mathbf{A} \mathbf{d} \mathbf{S}_{\alpha}^{\prime}$ is not globally hyperbolic (cf. Theorem 1.1.3. It is neither causal (quite obviously, there are closed time-like curves) nor does equation (1.1) hold. The first problem can be avoided by passing to the universal cover $\mathbf{A d S}_{\alpha}$, to which we refer as Anti de Sitter spacetime. However, it can be shown that the second problem cannot be avoided. Therefore, even $\mathbf{A d S}_{\alpha}$ is not globally hyperbolic. However, QFT in Anti de Sitter spacetime has received much attention for many years now, and this provides motivation to perform our investigation despite these 
causal pathologies. Moreover, due to its simple structure and its similarity to de Sitter spacetime, $\mathbf{A d S}_{\alpha}$ provides an interesting testing ground for the ideas discussed here 19

There are two kinds of (locally) time-like Killing vector fields in $\operatorname{AdS}_{\alpha}$. The first we call $L$; it is time-like everywhere and gives rise to the Killing flow

$$
\phi: t \mapsto\left(\begin{array}{ccc}
\cos (t) & \sin (t) & \\
-\sin (t) & \cos (t) & \\
& & \mathbb{1}_{3}
\end{array}\right)
$$

The corresponding trajectories are not geodesic except for $\gamma_{0}(t):=\phi_{t}(y)$ with $y:=$ $(0, \alpha, 0,0,0)$.

Stottmeister [Sto09] has computed the expectation value of the Wick square for $\beta$-KMS states with respect to this flow. His result is

$$
\begin{aligned}
\omega_{\beta}\left(: \phi^{2}:(x)\right)=\frac{1}{-g_{x}(L, L)} \cdot \frac{1}{4 \pi^{2}}\left(\sum_{n=0}^{\infty} \frac{n}{e^{n \beta}-1}+\right. & \left.\frac{1}{2} \sum_{n=1}^{\infty} \frac{1}{\cosh (\beta n)-1}\right) \\
& +\frac{1}{24 \pi^{2} \alpha^{2}}-\frac{12}{\alpha^{2}} \alpha_{0} .
\end{aligned}
$$

The series appearing in this formula tend to zero as $\beta \rightarrow \infty$, so the expectation value in the corresponding ground state reads

$$
\lim _{\beta \rightarrow \infty} \omega_{\beta}\left(: \phi^{2}:(x)\right)=\frac{1}{24 \pi^{2} \alpha^{2}}-\frac{12}{\alpha^{2}} \alpha_{0}
$$

Using $\tilde{\epsilon}_{a b}$, the dynamics of the local temperature 3.32 yields the constraint:

$$
\left(\frac{1}{-g_{x}(L, L)}+\frac{1}{\alpha^{2}}\right) \frac{1}{4 \pi^{2}}\left(\sum_{n=0}^{\infty} \frac{n}{e^{n \beta}-1}+\frac{1}{2} \sum_{n=1}^{\infty} \frac{1}{\cosh (\beta n)-1}\right)=0
$$

which shows that the ground state $\beta \rightarrow \infty$ is in local thermal equilibrium.

The second type of Killing fields is time-like only in wedge-shaped regions, similar to the ones appearing in de Sitter spacetime. Of the several possibilities, we focus our attention

\footnotetext{
${ }^{19}$ Given a lcQFT $\mathcal{A}$, there is a method of constructing an algebra of observables $\mathcal{A}(\mathbf{M})$ on a non-globally hyperbolic spacetime $\mathbf{M}$. It essentially requires that every point in $\mathbf{M}$ possesses a globally hyperbolic neighbourhood $\mathbf{N}$ (with some choice of time orientation) such that the subalgebra $\mathcal{A}(\mathbf{M} ; \mathbf{N})$ of $\mathcal{A}(\mathbf{M})$ consisting of field polynomials smeared with test functions with support in $\mathbf{N}$ coincides with the "intrinsic" algebra $\mathcal{A}(\mathbf{N})$, obtained by the functor $\mathcal{A}$. See [FH96] and references therein for details. We do not comment further on this approach, but assume that the free scalar quantum field and its Wick powers have been constructed in $\mathbf{A d S}_{\alpha}$, choosing "transparent" boundary conditions for the Green's operators of the field, see [Sto09] for discussion.
} 
on the Killing vector field $K_{2}$, for reasons of symmetry ${ }^{20} K_{2}$ induces the Killing flow

$$
\chi: \eta \mapsto\left(\begin{array}{llll}
\cosh (\eta) & & \sinh (\eta) & \\
& 1 & & \\
\sinh (\eta) & & \cosh (\eta) & \\
& & & \mathbb{1}_{2}
\end{array}\right)
$$

and it is positive time-like only in the wedge

$$
W_{2}^{+}=\left\{x \in A d S_{\alpha}|| x_{0} \mid<x_{2}\right\} .
$$

There are no geodesics for this flow. We consider the corresponding $\beta$-KMS states in the interior of $W_{2}^{+}$. Computing the expectation value of the Wick square, one finds ${ }^{21}$

$$
\omega_{\beta}\left(: \phi^{2}:(x)\right)=\frac{1}{-g_{x}\left(K_{2}, K_{2}\right)}\left(\frac{1}{12 \beta^{2}}-\frac{1}{12(2 \pi)^{2}}\right)+\frac{1}{24 \pi^{2} \alpha^{2}}-\frac{12}{\alpha^{2}} \alpha_{0} .
$$

Note that, apart from the + sign in the second term and the - sign in the third term, this expression has the same form as the corresponding expectation value 3.36 in the wedge region of de Sitter spacetime.

The evolution equation for the local temperature 3.32 yields the constraint:

$$
\left(\frac{1}{-g_{x}\left(K_{1}, K_{1}\right)}+\frac{1}{2 \alpha^{2}}\right)\left(\frac{1}{12 \beta^{2}}-\frac{1}{12(2 \pi)^{2}}\right)=0,
$$

which shows that the $\beta=2 \pi$ state is in LTE. This state corresponds to the $\beta \rightarrow \infty$ state for the dynamics induced by $L$ (i.e. the "vacuum" [BFS00]). ${ }^{22}$

Einstein Static spacetime The next example is Einstein static Universe E, which is an exact solution of the Einstein equations with cosmological constant $\Lambda>0$ and energy momentum tensor of a perfect fluid. It can be viewed as $\mathbb{R} \times S^{3}$, with metric

$$
\mathrm{d} s^{2}=\frac{1}{\Lambda}\left(-\mathrm{d} t^{2}+\mathrm{d} \chi^{2}+\sin ^{2}(\chi)\left(\mathrm{d} \theta^{2}+\sin ^{2}(\theta) \mathrm{d} \phi^{2}\right)\right),
$$

where we have set the sectional curvature equal to 1. Stottmeister [Sto09] has calculated the two-point function of $\beta$-KMS states with respect to the dynamics given by translations of $t$. His result for the expectation value of the Wick square in these states is

$$
\omega_{\beta}\left(: \phi^{2}:\right)=\frac{\Lambda}{4 \pi^{2}}\left(\sum_{n=0}^{\infty} \frac{n}{e^{\beta n}-1}+\frac{1}{2} \sum_{n=1}^{\infty} \frac{1}{\cosh (\beta n)-1}-\frac{1}{12}\right)+\alpha_{0} 6 \Lambda,
$$

\footnotetext{
${ }^{20}$ The symmetry group of $\mathbf{A d S _ { \alpha }}$ is the restriction of the symmetry group $O(2,3)$ of ambient $\mathbb{R}^{5}$ to $A d S_{\alpha}$. ${ }^{21}$ This result is new. However, we do not put the calculation on record here, because there are no new ideas involved. The methods used are those developed in [Sto09].

${ }^{22} \mathrm{Had}$ we used the original definition of the thermal energy tensor instead, the resulting constraint would have exactly the same undesirable form as the corresponding expression in de Sitter spacetime.
} 
which is constant over spacetime. The value in the corresponding ground state is [Sto09, eqn. (5.11)]

$$
\lim _{\beta \rightarrow \infty} \omega_{\beta}\left(: \phi^{2}:\right)=\left(-\frac{1}{288 \pi^{2}}+\alpha_{0}\right) 6 \Lambda,
$$

The Einstein static universe is locally conformally flat and $R=6 \Lambda$ is constant, moreover $R^{\alpha \beta} R_{\alpha \beta}=12 \Lambda^{2}$, which shows that there is no anomaly. However, equation (3.31) still has a rather undesirable form, while using the modified thermal energy tensor $\tilde{\epsilon}_{a b}$ instead shows that all of the KMS states are in LTE, because $\omega_{\beta}\left(: \phi^{2}:\right)$ is constant.

Discussion We would like to draw some conclusions from the previous examples at this point. The first point concerns the choice of $\alpha_{0}$, the renormalization of the Wick square. We propose the choice

$$
\alpha_{0}=\frac{1}{288 \pi^{2}},
$$

such that $: \phi^{2}$ : is a conformally covariant quantum field (see last section). With this choice, the Gibbons Hawking state in de Sitter, the Unruh Hawking type state in Anti de Sitter (i.e. $\beta=2 \pi$ for the $K_{2}$-induced dynamics and the ground state for the $L$-induced dynamics) and the ground state in Einstein static spacetime all have a local temperature of zero. This is not surprising, since all of these states are conformal vacua [BD84] in their respective spacetimes. This then establishes a close analogy to the Rindler case, where the Unruh state also has local temperature zero, regardless of the choice of renormalization constant.

Buchholz and Schlemmer [BS07] have proposed choosing $\alpha_{0}$ such that, in static de Sitter spacetime (cf. equation (3.36), the ground state $\beta \rightarrow \infty$ has local temperature zero on the geodesic, where $-g\left(K_{1}, K_{1}\right)=\alpha^{2} \cdot{ }^{23}$ The motivation ${ }^{24}$ behind this choice is that, while the time-like Killing trajectories in $\mathbf{d} \mathbf{S}_{\alpha \text {,stat }}$ are geometrically quite unlike time translations in Minkowski spacetime, at least on the geodesic, the KMS states should behave like reference states when tested with scalar observables. The corresponding choice is $\alpha_{0}^{B S}=\frac{1}{192 \pi^{2}}$. However, the argument of Buchholz and Schlemmer only works in de Sitter spacetime: it can be seen that with $\alpha_{0}^{B S}$, for Anti de Sitter (cf. equations 3.44) and (3.47), the Unruh Hawking type state is not in LTE (not even on the geodesic). What is more, in Einstein static spacetime (cf. equation (3.50), the ground state is also out of local equilibrium everywhere. In other words, except for de Sitter spacetime, all the conformal vacua in the curved spacetime examples discussed so far fail to be in LTE, even on the geodesics (if present).

Having knowledge of all the other examples, it seems unnatural to justify a choice of $\alpha_{0}$ based only on the geodesics in de Sitter spacetime. We therefore introduce the

\footnotetext{
${ }^{23}$ On the geodesic, the inverse local temperature then agrees with the KMS parameter for all KMS states.

${ }^{24}$ I would like to thank J. Schlemmer for discussions on this point.
} 
conformal renormalization here - conformal vacua should have a local temperature of zero. ${ }^{25}$ This choice, equation (3.51), entails an interesting relation between the KMS parameter $T_{\mathrm{KMS}}=\beta^{-1}$ and the local temperature in the wedges of de Sitter and Anti de Sitter spacetime. If $T_{\mathrm{KMS}}(x)$ denotes the KMS parameter, corrected for the Tolman redshift, equation (3.38), and $T(x)$ is the local temperature as measured by the Wick square, then it is easy to see that ${ }^{26}$

$$
T_{\mathrm{KMS}}(x)=\sqrt{ \pm \frac{1}{(2 \pi \alpha)^{2}}+\frac{a^{2}(x)}{(2 \pi)^{2}}+T^{2}(x)},
$$

where the + sign holds for de Sitter, while the - sign holds for Anti de Sitter. In fact, this relation also holds for Rindler spacetime, if one takes into account that the first term under the square root is absent in that case.

This formula underlines an important point raised before: there are several components which combine in order to render a curved spacetime state KMS and hence passive with regard to the dynamics in question. Here, this is embodied by the fact that the KMS parameter with respect to which the state is passive decomposes into a geometric contribution due to spacetime curvature (corresponding to the first term under the square root), a part which encodes the effect of acceleration (the second term) and, finally, a contribution due to local thermality (the third term).

Note that equation 3.52 is a generalization of formulas for the Unruh-Hawking states first found and discussed in [NPT96, DL97, Jac98]. In [BS07], a different formula for de Sitter spacetime was presented based on their choice of $\alpha_{0}$ (their eqn. (11)). In our view, however, formula 3.52 is favourable, since it displays a contribution due to the curvature, which should play a rôle in the passivity and KMS property of states in curved spacetime.

At this point we would like to comment on the meaning of conformally covariant local thermometers. The measurement of global temperature usually requires procedures which break the conformal symmetry, because a time scale, and hence an energy scale, needs to be fixed in advance, while a conformally covariant local thermometer means that the local temperature scales with the conformal factor. This should be seen as an indication that additional thermal observables are required in order to determine other local thermal

\footnotetext{
${ }^{25}$ We attach more significance here to the local thermality of the highly symmetric Hawking-Unruh type states than to ground states with respect to wedge dynamics as in Rindler, de Sitter and Anti de Sitter spacetime. In fact, these particular ground states are out of local equilibrium with our choice of $\alpha_{0}$. ${ }^{26}$ In Anti de Sitter spacetime, the relation corresponding to 3.37 reads
}

$$
\frac{1}{-g_{x}\left(K_{1}, K_{1}\right)}=a^{2}(x)-\frac{1}{\alpha^{2}} .
$$

Therefore, the acceleration is bounded below and the sum of the first two terms on the right hand side of 3.52 is always positive in the wedge. 
functions in conjunction with $T(x)$ - such as the thermal energy tensor, which is not conformally covariant. We comment on how our choice of renormalization constants can be generalized to theories which are not conformally covariant, such as the massive scalar field, in Condition 2

The second point we wish to discuss concerns the thermal energy tensor. We realize that the original definition of the thermal energy tensor $\epsilon_{a b}$ in curved spacetime, equation 3.21, leads to very strict constraints in the KMS states, which for example entail that the conformal vacua are not $\mathbf{T}^{(2)}$-thermal anywhere (except, of course, in Rindler spacetime). However, due to high degree of symmetry in these states, we expect some degree of thermality, also with regard to their thermal energy. While for the Wick square, we could choose $\alpha_{0}$ accordingly, for the thermal energy tensor nothing can be done using the renormalization freedom in the spacetimes with $\square R=0$. However, we have seen that if the modified thermal energy tensor $\tilde{\epsilon}_{a b}$ is used instead, see equation 3.24 , the conformal vacua are indeed locally thermal.

We emphasize again that we have fixed the renormalization and energy momentum tensor by testing KMS states in some particularly symmetric curved spacetimes, but these choices in fact apply to all spacetimes. From now on, we use conformal renormalization and the modified stress energy tensor.

Definition 3.2.1. In curved spacetime, the Wick square, serving as a local thermometer, is the locally conformally covariant quantum field of weight 2 which is given by

$$
: \phi^{2}:=: \phi^{2}:_{h}+\frac{R}{288 \pi^{2}} .
$$

The thermal energy tensor is given by

$$
\begin{aligned}
\tilde{\epsilon}_{a b} & :=-: \phi\left(\nabla_{a} \nabla_{b} \phi\right):+\frac{1}{4} \nabla_{a} \nabla_{b}: \phi^{2}:+\frac{1}{6} R_{a b}: \phi^{2}:-\frac{1}{4} g_{a b}: \phi P \phi: \\
& =\tilde{\epsilon}_{h, a b}-\frac{1}{12}\left(\nabla_{a} \nabla_{b}-g_{a b} \square\right) \frac{R}{288 \pi^{2}}+T_{a b}^{R F}-\frac{g_{a b}}{4}\left(T^{R F}\right)^{a}{ }_{a},
\end{aligned}
$$

where $\tilde{\epsilon}_{h, a b}$ includes only Hadamard normal ordered Wick products.

Recall that the renormalization constants $\beta_{1}$ and $\beta_{2}$ enter the definition of $\tilde{\epsilon}_{a b}$ via $T_{a b}^{R F}$, cf. equation (1.39). We test our choices in two more examples of physical interest.

Cosmological Spacetime Our next example is of importance in cosmology. According to the standard paradigm, on large scales, the universe can be described by a homogeneous and isotropic solution of Einstein's equations. The corresponding class of models is given by the Friedmann-Robertson-Walker spacetimes. The only degree of freedom in the metric of these spacetimes is the scale factor $a(t)$, where $t$ denotes the cosmological time, which ranges over an open interval $I \subset \mathbb{R}$. Recent measurements of the evolution of the Hubble 
function suggest that a model with flat spatial sections is appropriate, in contrast to spherical or hyperbolic models.

A spatially flat Friedmann-Robertson-Walker spacetime $\mathbf{F}_{a}$ is given by $I \times \mathbb{R}^{3}$ with metric

$$
g_{\mathbf{F}_{a}}=-\mathrm{d} t^{2}+a^{2}(t)\left(\mathrm{d} x^{2}+\mathrm{d} y^{2}+\mathrm{d} z^{2}\right) .
$$

Introducing the conformal time $\tau$ from the differential relation $\mathrm{d} \tau:=\frac{\mathrm{d} t}{a(t)}$ and writing $a(\tau):=a(t(\tau))$, the metric becomes

$$
g_{\mathbf{F}_{a}}=a^{2}(\tau)\left(-\mathrm{d} \tau^{2}+\mathrm{d} x^{2}+\mathrm{d} y^{2}+\mathrm{d} z^{2}\right),
$$

which shows that $\mathbf{F}_{a}$ is conformally embedded into Minkowski spacetime. Clearly, the conformal embedding $\psi: \mathbf{F}_{a} \hookrightarrow \mathbf{M}_{0}$ is given by $\psi(\tau, \vec{x})=(\tau, \vec{x})$ and the conformal factor is $\Omega(\psi(\tau, \vec{x}))=1 / a(\tau)$.

One can now introduce so-called conformal KMS states for the massless, conformally coupled field by using KMS states in Minkowski spacetime. ${ }^{27}$ Using $e=\partial_{\tau} \in V^{+}$we use the KMS state $\omega_{\beta}^{0}$ in Minkowski spacetime to define the state

$$
\omega_{\beta}=\omega_{\beta}^{0} \circ \alpha_{\psi}
$$

in $\mathbf{F}_{a}$, which is Hadamard [Pin09, DHP11].

Since the Wick square is a conformally covariant quantum field, it is easy to see that

$$
\omega_{\beta}\left(: \phi^{2}:(\tau)\right)=\frac{1}{12 a^{2}(\tau) \beta^{2}},
$$

which means that the inverse local temperature in these states scales with $a(\tau)$, i.e. $\beta(\tau)=$ $a(\tau) \beta$, which is expected from a conformally covariant theory in zeroth order cosmology.

In the states $\omega_{\beta}$, the dynamics of the local temperature 3.32 results in the condition

$$
\ddot{a}=0
$$

for all $\beta$. Here, 'denotes the derivative with respect to cosmological time ${ }^{28}$ Depending on the initial conditions, this condition can accommodate power law inflation and also Minkowski spacetime. This result shows that only in a special cosmology, the conformal KMS states can be in LTE with respect to the thermal energy tensor. Of course, the model of the massless, conformally coupled free scalar field is too simple to be able to fully

\footnotetext{
${ }^{27}$ This works in the same way as for conformal vacua, as discussed in [BD84]. Note that conformal KMS states are well known and recently appeared as special cases of the approximate KMS states defined in [DHP11].

${ }^{28} \mathrm{Had}$ we used $\epsilon_{a b}$ instead of $\tilde{\epsilon}_{a b}$, the resulting equation would be a non-linear third order differential equation in the Hubble parameter $H:=\dot{a} / a$, depending on the inverse temperature $\beta$ and a linear combination of the renormalization constants $\beta_{1}$ and $\beta_{2}$.
} 
describe quantum effects in the early universe. However, our discussion shows that linear constraints arising from the LTE condition and equations of state might have a bearing on cosmological parameters in more realistic models. See also [Ver11] for a discussion on LTE in cosmology.

Schwarzschild spacetime Our last example is Schwarzschild spacetime $\mathbf{S}_{M}$ surrounding a static black hole of mass $M . \mathbf{S}_{M}$ proves to be an interesting testing ground for LTE, since it is a curved spacetime which is not conformally flat (in contrast to our previous examples) and is a vacuum solution to Einstein's equations. The latter point implies that the renormalization ambiguity of the Wick square is not present, as $R=0$.

This paragraph is based on an interesting early attempt to define local temperature in QFT by measuring the Wick square, namely Page's work in the eighties Pag82.29 The author calculates the expectation value of the Wick square and the energy momentum tensor in thermal states for the conformally coupled free scalar field in backgrounds with static Einstein metric 30 , where he uses the so-called Bekenstein-Parker Gaussian approximation 31

Exterior Schwarzschild spacetime can be covered by coordinates $(t, r, \theta, \varphi) \in \mathbb{R} \times$ $(2 M, \infty) \times S^{2}$ and the metric is given by

$$
g=-\left(1-\frac{2 M}{r}\right) \mathrm{d} t^{2}+\left(1-\frac{2 M}{r}\right)^{-1} \mathrm{~d} r^{2}+r^{2}\left(\mathrm{~d} \theta^{2}+\sin (\theta)^{2} \mathrm{~d} \varphi^{2}\right),
$$

which is obviously static with time-like Killing vector $K:=\partial_{t}$. Schwarzschild spacetime, as a vacuum solution, is an Einstein spacetime with $\Lambda=0$. Page thus used his result to compute the Wick square in the Hartle Hawking state, which is KMS with respect to the dynamics induced by $K$, at the Hawking Temperature $\beta=8 \pi M$ [Wa194]. The Wick square in this state takes the value

$$
\omega_{8 M \pi}\left(: \phi^{2}:(x)\right) \approx \frac{1}{12(2 \pi)^{2}}\left(1+\frac{2 M}{r}+\left(\frac{2 M}{r}\right)^{2}+\left(\frac{2 M}{r}\right)^{3}\right),
$$

which approaches $\frac{1}{12(2 \pi)^{2}}$ for $r \rightarrow \infty$, i.e. the inverse local temperature of $\omega_{8 M \pi}$ at infinity is $\beta(\infty)=2 \pi$.

Turning to the thermal energy tensor and applying equation 3.32 to 3.59 yields the constraint

$$
1-\frac{4 M}{r}-\frac{36 M^{2}}{r^{2}} \approx 0
$$

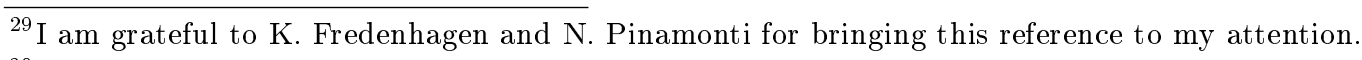

${ }^{30}$ Recall that a spacetime $\mathbf{M}$ with metric $g$ is called Einstein, if there is a constant $\Lambda \in \mathbb{R}$ such that $R_{a b}=\Lambda g_{a b}$. All of the previous examples are Einstein spacetimes, except for Robertson Walker spacetimes with non-constant $a(\tau)$.

${ }^{31}$ The corresponding approximation was slightly improved in [CH84], but this does not change our discussion here. 
which entails that only at $r \approx 9 M$ can the Hartle Hawking state be in LTE. ${ }^{32}$ This result shows that even for the modified thermal energy tensor, the Hartle Hawking state is almost nowhere in LTE. However, this is not unexpected: as we have mentioned several times before, there is no reason to expect that KMS states in curved spacetime have a high degree of local thermality, especially with respect to non-scalar observables. These simply measure the degree to which the state resembles a reference state.

We can interpret the result for the Wick square as follows: for the Hartle Hawking state at spatial infinity, the local temperature agrees with the Tolman redshifted KMS Temperature, up to a factor of $\frac{1}{4 M}$ (the surface gravity of exterior Schwarzschild spacetime). This resembles the situation for the Unruh state in Rindler spacetime, where at spatial infinity the Tolman shifted KMS temperature is zero, agreeing with the local temperature, cf. equation 3.34. Recall that for both spacetimes, these findings are not influenced by the choice of $\alpha_{0}$. At spatial infinity, in both spacetimes, the trajectories with respect to which the states in question are KMS resemble inertial time-like trajectories in Minkowski spacetime (here, the asymptotic flatness of Schwarzschild spacetime comes into play). These results are therefore consistent.

Choosing coordinates appropriate for the "wedge-dynamics" induced by $K_{2}$ in Anti de Sitter spacetime, one can show that the Tolman redshifted KMS temperature for the Hawking-Unruh type state $(\beta=2 \pi)$ tends to zero at infinity (which is time-like for Antide Sitter). This can be seen as a confirmation of our choice of renormalization constant $\alpha_{0}$, because the local temperature in this state is zero at infinity (in fact, everywhere). This therefore establishes an analogy to the Schwarzschild and Rindler spacetime and provides support for our choice of $\alpha_{0}$.

In general, when there is no conformal symmetry available, like in the massive case, we propose the following prescription for fixing the corresponding renormalization constants. This requirement supplements Condition 1 in Section 2.2.1 as criterion for the choice of thermal observables.

Condition 2. Let there be given KMS states for a Killing flow for which there exist sequences of Killing trajectories, which in some limit resemble inertial trajectories in Minkowski spacetime. In that limit the inverse local temperature must resemble the Tolman redshifted KMS parameter for Hawking-Unruh type states in spacetimes where the latter exist.

We also want to reiterate an important point: as the examples have shown, KMS states in curved spacetime do not always possess a local temperature, i.e. in certain regions and for certain ranges of the corresponding KMS parameter, these states are out of local

\footnotetext{
${ }^{32}$ We note that there exist time-like geodesics with closed orbits at that radius.
} 
equilibrium even with regard to the Wick square. However, our abstract results in Chapter 2 and also Corollary 3.1.6 allow us to draw the conclusion that even in such regions there are states which are $\mathbf{T}_{2 n}(x)$-thermal for any $n$. Here, we display two results of this type.

Proposition 3.2.2. Let $\mathcal{O} \subset M$ be a compact subset of some spacetime $\mathbf{M}$ and let $n \in \mathbb{N}$. If the Wick powers are chosen such that $C_{2 m}=(2 m-1) ! ! \alpha_{0}^{m} R^{m}$ for all $m=1,2, \ldots, n$, then there are (not necessarily quasi-free) $\mathbf{T}_{2 n}(\mathcal{O})$-thermal states in $\mathcal{S}(\mathbf{M})$.

Proof. A state is $\mathbf{T}_{2}(x)$-thermal if the corresponding expectation value of $: \phi^{2}:(x)$ is positive. We can construct a state with this property for all $x \in \mathcal{O}$ as follows. We pick any state $\omega \in \mathcal{S}(\mathbf{M})$ with non-vanishing one-point function $\omega^{(1)}$ and modify its two-point function $\omega^{(2)}$ by adding another term

$$
\tilde{\omega}_{c}^{(2)}:=\omega^{(2)}+c \omega^{(1)} \otimes \omega^{(1)}
$$

with some $c \in \mathbb{R}$. Recall that since $\omega \in \mathcal{S}(\mathbf{M})$, the $\omega^{(1)}$ are smooth. $\tilde{\omega}_{c}^{(2)}$ is a valid two-point function and we can consider the corresponding quasi-free state $\tilde{\omega}_{c}$. Clearly

$$
\tilde{\omega}_{c}\left(: \phi^{2}:(x)\right)=\omega\left(: \phi^{2}:(x)\right)+c\left(\omega^{(1)}(x)\right)^{2} .
$$

Due to the compactness of $\mathcal{O}$, we may choose the constant $c$ such that $\tilde{\omega}_{c}\left(: \phi^{2}:(x)\right)>0$ for all $x \in \mathcal{O}$. There are obviously many states which satisfy this inequality and are consequently $\mathbf{T}_{2}(\mathcal{O})$-thermal. By Corollary 3.1.6 this means that these states, being quasi-free, are $\mathbf{T}_{2 n}(\mathcal{O})$-thermal as well.

Now we use the fact that $\mathbf{T}_{2 n}(\mathcal{O})$-thermality is stable under convex combinations. We simply mix the so-constructed quasi-free states and thus construct states which are not quasi-free but still $\mathbf{T}_{2 n}(\mathcal{O})$-thermal.

The method discussed in Section 2.2.3 provides an alternative way to show the existence of $\mathbf{T}_{2 n}(x)$-thermal states for $x \in \mathbf{M}$. Namely, the results in Chapter 2 entail that there exist $\mathbf{T}_{2 n}(x)$-thermal states if any linear combination

$$
\psi(x):=\sum_{i=0}^{n} c_{i}: \phi^{2 i}:(x), \quad c_{i} \in \mathbb{R},
$$

has a positive scaling limit, cf. Proposition 2.2.9.

Lemma 3.2.3. The scaling limit of a quasi-free Hadamard state $\omega \in \mathcal{S}(\mathbf{M})$ at $x \in \mathbf{M}$ exists for the Wick powers : $\phi^{2 n}:$ for all $n \in \mathbb{N}$. The scaling limit two-point function is non-trivial and the dimension of the field is $2 n 33$

\footnotetext{
${ }^{33}$ Since derivatives only increase the dimension, we note that a similar result holds for differentiated Wick powers : $\left(\nabla^{o(1)} \phi\right)\left(\nabla^{o(2)} \phi\right) \ldots\left(\nabla^{o(n)} \phi\right)$ :.
} 
Proof. One starts by computing the $m$-point functions of $\omega$ with respect to the field : $\phi^{2 n}$ :, making use of Wick's theorem 1.16. On finds a sum of expectation values of certain Wick powers in $\omega$, the highest of which is : $\phi^{2 n m}$ :. The most singular term is thus the one given by a product of $n m$ Hadamard parametrices $h$. Therefore, we only need to know the scaling limit of $h$, but this follows from [SV01, Lemma A.3]. One finds that the scaling function for the Wick powers : $\phi^{2 n}$ : is given by $N(\lambda)=\lambda^{2 n-4}$, i.e. $d=2 n$. We omit the details of the calculation.

This lemma, together with Wick's theorem 1.16, allows us to compute the scaling limit of the field $\psi$ in some quasi-free Hadamard state. As there are no relations in the limit and no fields sharing the same dimension (see the discussion in Section 2.2.3), we deduce that the scaling limit is determined by the highest Wick power which appears in the linear combination $\psi$ and is thus positive. By Proposition 2.2.9, all elements of $\operatorname{span}\left\{\mathbf{T}_{2 n}\right\}$ are therefore unbounded and Proposition 2.2.7 allows us to conclude that there are many $\mathbf{T}_{2 n}(x)$-thermal states. $\mathbf{T}_{2 n}$-thermality pertains in a neighbourhood of $x$, because there are no linear constraints, see the argument at the end of Section 2.2.2. 


\section{Conclusion \& Perspectives}

In this thesis, we were concerned with the conceptual question under which circumstances states in quantum field theory in curved spacetime are in thermal equilibrium and when it is therefore meaningful to attach local thermal parameters to them. For the purpose of illustrating our results, we have predominantly considered the massless, conformally coupled, free scalar field. However, most of our conclusions also hold in realistic models and it would be worthwhile to consider LTE states in perturbative QFT, for example.

We have given arguments explaining that thermal observables do not form a vector space in curved spacetime and, moreover, must be chosen to be linearly independent 1 We have seen that in certain cases there are relations between corresponding local thermal parameters which are induced by linear equations of state. It is interesting that these relations lead to evolution equations for the local thermal parameters.

Choosing thermal observables for which no such relations hold, we have shown that there exist states to which the corresponding local thermal parameters can be assigned in bounded regions of Minkowski spacetime. Moreover, with a natural assumption regarding the numerical range of the thermal observables, there exist states which are in equilibrium at any given point in a curved spacetime. Again, in the absence of linear constraints, the thermality of these states extends to neighbourhoods.

Furthermore, we have argued that KMS states in stationary curved spacetimes, though passive with respect to certain observers, may not be viewed in analogy to global thermal equilibrium states in Minkowski spacetime. Indeed, not all of these states are in local equilibrium. For those that are, the parameter $\beta$ cannot necessarily be interpreted as an inverse temperature. In fact, $\beta$ decomposes into contributions from the curvature, the acceleration of the observer and the local temperature. Usage of the thermal observables to obtain information on the KMS states in curved spacetime also allowed us to fix the renormalization freedom exhibited by these fields in terms of physically meaningful parameters.

Finally, we wish to point out possible directions for further research. Regarding our existence proof for LTE states in Minkowski spacetime, it would be interesting to clarify whether it can be extended to thermal observables for which there are constraints arising

\footnotetext{
${ }^{1}$ Taking advantage of the freedom inherent to the choice of thermal observables in curved spacetime, in some cases it is possible to recover a linear structure on these sets.
} 
from linear equations of state. Additionally, a deeper understanding of the requirements for local equilibrium in regions of curved spacetime is, of course, desirable. In particular, this involves showing that in case of linear constraints, there are states which satisfy the resulting evolution equations in open regions in curved spacetime. While we have seen that these evolution equations are satisfied in some KMS states in certain spacetimes, a more general result has yet to be obtained. 


\section{Bibliography}

[Ara00] H. Araki, Mathematical Theory of Quantum Fields, Oxford University Press, 2000.

[Bah06] B. Bahr, The Hot Bang State of massless Fermions, Lett.Math.Phys (2006), no. $78,39-54$.

[BD84] N. D. Birrell and P. Davies, Quantum Fields in Curved Space, Cambridge University Press, 1984.

[BEE96] J. K. Beem, P. Ehrlich, and K. Easley, Global Lorentzian Geometry, 2nd ed., Pure and Applied Mathematics, CRC Press, 1996.

[BF00] R. Brunetti and K. Fredenhagen, Microlocal Analysis and Interacting Quantum Field Theories: Renormalization on Physical Backgrounds, Comm. Math. Phys. 208 (2000), 623-661.

[BF09] C. Bär and K. Fredenhagen, Quantum Field Theory on Curved Spacetime: Concepts and Mathematical Foundations, Lecture Notes in Physics, no. 786, Springer Heidelberg Berlin, 2009.

[BFK96] R. Brunetti, K. Fredenhagen, and M. Köhler, The microlocal spectrum condition and Wick polynomials of free fields on curved spacetimes, Comm. Math. Phys. 180 (1996), no. 3, 633-652.

[BFS00] D. Buchholz, M. Florig, and S. J. Summers, Hawking-Unruh temperature and Einstein causality in anti-de Sitter space-time, Class. Quant. Grav. 17 (2000), L31-L37.

[BFV03] R. Brunetti, K. Fredenhagen, and R. Verch, The Generally Covariant Locality Principle - A New Paradigm for Local Quantum Field Theory, Comm. Math. Phys. 237 (2003), 31-68.

[BGP07] C. Bär, N. Ginoux, and F. Päffle, Wave equations on Lorentzian manifolds and quantization, ESI Lectures in Mathematics and Physics, European Mathematical Society Publishing House, 2007. 
[BOR02] D. Buchholz, I. Ojima, and H. Roos, Thermodynamic Properties of Nonequilibrium States in Quantum Field Theory, Ann. Physics 297 (2002), 219242.

[Bos00] H. Bostelmann, Lokale Algebren und Operatorprodukte am Punkt, Ph.D. thesis, Universität Göttingen, 2000.

[BR96] O. Bratelli and D.W. Robinson, Operator algebras and quantum statistical mechanics. Vol. 2: Equilibrium states. Models in quantum statistical mechanics, Springer Berlin, Germany, 1996.

[BR07] R. Brunetti and G. Ruzzi, Superselection sectors and general covariance I, Comm. Math. Phys. 270 (2007), 69-108.

[BR09] — Quantum charges and spacetime topology: The emergence of new superselection sectors, Comm. Math. Phys. 287 (2009), 523-563.

[BS05] A. N. Bernal and M. Sánchez, Smoothness of Time Functions and the Metric Splitting of Globally Hyperbolic Spacetimes, Comm. Math. Phys. 257 (2005), $43-50$.

[BS07] D. Buchholz and J. Schlemmer, Local Temperature in Curved Spacetime, Classical Quantum Gravity 24 (2007), F25-F31.

[Buc03] D. Buchholz, On Hot Bangs and the Arrow of Time in Relativistic Quantum Field Theory, Comm. Math. Phys. 237 (2003), 271-288.

[CF09] B. Chilian and K. Fredenhagen, The Time Slice Axiom in Perturbative Quantum Field Theory on Globally Hyperbolic Spacetimes, Comm. Math. Phys. 287 (2009), 513-522.

[CH84] P. Candelas and K. W. Howard, Vacuum $\left\langle\varphi^{2}\right\rangle$ in Schwarzschild spacetime, Phys. Rev. D 29 (1984), 1618-1625.

[Coh80] D.L. Cohn, Measure Theory, Birkhäuser, Boston, 1980.

[DB60] B.S. DeWitt and R.W. Brehme, Radiation Damping in a Gravitational Field, Ann. Phys. 9 (1960), 220.

[DF01] M. Dütsch and K. Fredenhagen, Perturbative algebraic field theory, and deformation quantization, Mathematical Physics in Mathematics and Physics: Quantum and Operator Algebraic Aspects (R. Longo, ed.), Fields Institute Communications, Providence, RI, 2001. 
[DF06] Y. Décanini and A. Folacci, Off-diagonal coefficients of the DeWitt-Schwinger and Hadamard representations of the Feynman propagator, Phys. Rev. D 73 (2006), no. 4.

[DFP08] C. Dappiaggi, K. Fredenhagen, and N. Pinamonti, Stable cosmological models driven by a free quantum scalar field, Phys. Rev. D77 (2008).

[DHMP10] C. Dappiaggi, T.-P. Hack, J. Möller, and N. Pinamonti, Dark Energy from Quantum Matter, arXiv:1007.5009v1 [astro-ph.CO], 2010.

[DHP11] C. Dappiaggi, T.-P. Hack, and N. Pinamonti, Approximate KMS states for scalar and spinor fields in Friedmann-Robertson-Walker spacetimes, Annales Henri Poincare 12 (2011), 1449-1489.

[Dim80] J. Dimock, Algebras of Local Observables on a Manifold, Comm. Math. Phys. 77 (1980), 219-228.

[DL97] S. Deser and O. Levin, Accelerated detectors and temperature in (anti-) de Sitter spaces, Class. Quant. Grav. 14 (1997), 163-168.

[DMP09] C. Dappiaggi, V. Moretti, and N. Pinamonti, Distinguished quantum states in a class of cosmological spacetimes and their Hadamard property, J. Math. Phys. 50 (2009).

[DV10] A. Degner and R. Verch, Cosmological particle creation in states of low energy, Journal of Mathematical Physics 51 (2010).

[EGJ65] H. Epstein, V Glaser, and A. Jaffe, Nonpositivity of the Energy Density in Quantized Field Theories, Nuovo Cimento (1965), no. 36, 1016-1022.

[Few05] C.J. Fewster, Energy Inequalities in Quantum Field Theory, ArXiv: math$\mathrm{ph} / 0501073 \mathrm{v} 1,2005$.

[Few07] C. J. Fewster, Quantum energy inequalities and local covariance II: categorical formulation, Gen. Rel. Grav. 39 (2007), 1855-1890.

[Few11] C.J. Fewster, On the notion of "the same physics in all spacetimes", Quantum Field Theory and Gravity. Conceptual and mathematical advances in the search for a unified framework. (F. Finster, O. Müller, M. Nardmann, J. Tolksdorf, and E. Zeidler, eds.), Birkhäuser, 2011.

[FH81] K. Fredenhagen and J. Hertel, Local algebras of observables and pointlike localized fields, Comm. Math. Phys. 80 (1981), 555-561. 
[FH87] K. Fredenhagen and R. Haag, Generally covariant quantum field theory and scaling limits, Comm. Math. Phys. 108 (1987), 91-115.

[FH96] C.J. Fewster and A. Higuchi, Quantum field theory on certain nonglobally hyperbolic space-times, Class. Quant. Grav. 13 (1996), 51-62.

[FNW81] S.A. Fulling, F.J. Narcowich, and R.M. Wald, Singularity Structure of the Two Point Function in Quantum Field Theory in Curved Space-Time II, Annals Phys. (1981), no. 136, 243.

[FP06] C. J. Fewster and M. J. Pfenning, Quantum energy inequalities and local covariance. I. Globally hyperbolic spacetimes, Journal of Mathematical Physics 47 (2006).

[Ful89] S. A. Fulling, Aspects of Quantum Field Theory in Curved Spacetime, Cambridge University Press, 1989.

[FV11] C. J Fewster and R. Verch, Dynamical locality and covariance: What makes a physical theory the same in all spacetimes?, ArXiv: math-ph/1106.4785, 2011.

[Haa96] R. Haag, Local Quantum Physics, 2nd ed., Springer, Berlin, 1996.

[Hac10] T. P. Hack, On the Backreaction of Scalar and Spinor Quantum Fields in Curved Spacetimes, Ph.D. thesis, Universität Hamburg, 2010.

[Haw75] S.W. Hawking, Particle Creation by Black Holes, Comm. Math. Phys. 43 (1975), 199-220.

[HB74] R. Haag and Trych-Pohlmeyer B., Stability and equilibrium states, Comm. Math. Phys. 38 (1974), no. 3, 173-193.

[HE73] S. W. Hawking and G. F. Ellis, The large scale structure of space-time, University Press, Cambridge [Eng.], 1973.

[HHW67] R. Haag, N. Hugenholtz, and M. Winnik, On the equilibrium states in quantum statistical mechanics, Comm. Math. Phys. (1967), no. 5, 215-236.

[HL10] S. Hollands and G. Leiler, On the derivation of the Boltzmann equation in quantum field theory: Flat spacetime, ArXiv: cond-mat.statmech/1003.1621v1, 2010.

[Hör90] L. Hörmander, The Analysis of Linear Partial Differential Operators I, Springer, 1990. 
[Hör94] — Notions of Convexity, Birkhäuser, 1994.

[HR02] S. Hollands and W. Ruan, The State Space of Perturbative Quantum Field Theory in Curved Spacetimes, Ann. Henri Poincaré 3 (2002), 635-657.

[Hüb05] R. Hübener, Lokale Gleichgewichtszustände massiver Bosonen, Diploma Thesis, Universität Göttingen, 2005.

[HW01] S. Hollands and R. M. Wald, Local Wick Polynomials and Time Ordered Products of Quantum Fields in Curved Spacetime, Comm. Math. Phys. 223 (2001), 289-326.

[HW02] _ Existence of Local Covariant Time Ordered Products of Quantum Fields in Curved Spacetime, Comm. .Math. Phys. 231 (2002), 309-345.

[HW05]__ Conservation of the Stress Tensor in Perturbative Interacting Quantum Field Theory in Curved Spacetimes, Reviews in Mathematical Physics 17 (2005), 227-311.

[HW10] S. Hollands and R.M. Wald, Axiomatic Quantum Field Theory in Curved Spacetime, Comm. Math. Phys. 293 (2010), 85-125.

[IZ80] C. Itzykson and J.B. Zuber, Quantum Field Theory, McGraw-Hill Inc., New York, 1980.

[Jac98] T. Jacobson, Comment on: Accelerated detectors and temperature in (anti-) de Sitter spaces, Class. Quant. Grav. 15 (1998), 251-253.

[KFGV77] A. Kossakowski, A. Frigerio, V. Gorini, and M. Verri, Quantum Detailed Balance and KMS Condition, Comm. Math. Phys. 57 (1977), 97-110.

[KT90] E.W. Kolb and M.S. Turner, The early universe, Frontiers in Physics, AdisonWesley Publishing Company, 1990.

[Küs08] M. Küskü, A class of almost equilibrium states in Robertson-Walker spacetimes, Ph.D. thesis, Universität Hamburg, 2008.

[KW91] B. S. Kay and R. M. Wald, Theorems on the uniqueness and thermal properties of stationary, nonsingular, quasifree states on spacetimes with a bifurcate killing horizon, Phys. Rep. 207 (1991), 49-136.

[Mor03] V. Moretti, Comments on the Stress-Energy Tensor Operator in Curved Spacetime, Comm. Math. Phys. 232 (2003), 189-221. 
[MTW73] C. Misner, K. Thorne, and J. Wheeler, Gravitation, W. H. Freeman, 1973.

[NPT96] H. Narnhofer, I. Peter, and W. Thirring, How hot is the de Sitter space?, Int. J. Mod. Phys. B 10 (1996), 1507-1520.

[Olb07] H. Olbermann, States of Low Energy on Robertson-Walker Spacetimes, Class. Quant. Grav. 24 (2007), 5011-5030.

[O'N83] B. O’Neill, Semi-Riemannian Geometry With Applications to Relativity, Pure and Applied Mathematics, Academic Press, 1983.

[Pag82] D. N. Page, Thermal stress tensors in static Einstein spaces, Phys. Rev. D 25 (1982), 1499-1509.

[Par69] L. Parker, Quantized Fields and Particle Creation in Expanding Universes. I, Phys. Rev. 183 (1969), 1057-1068.

[Pet07] R. Petersen, Lokale Temperaturen auf gekrümmten Raumzeiten, Diploma Thesis, Universität Hamburg, 2007.

[Pin09] N. Pinamonti, Conformal Generally Covariant Quantum Field Theory: The Scalar Field and its Wick Products, Comm. Math. Phys. 288 (2009), 11171135.

[PPV11] E. Poisson, A. Pound, and I. Vega, The Motion of Point Particles in Curved Spacetime, Living Reviews in Relativity 14 (2011), no. 7.

[PW78] W. Pusz and S.L. Woronowicz, Passive states and KMS states for general quantum systems, Comm. Math. Phys. 58 (1978), no. 3, 273-290.

[Rad96a] M.J. Radzikowski, A local to global singularity theorem for quantum field theory on curved space-time, Comm. Math. Phys. 180 (1996), 1.

[Rad96b]_, Micro-Local Approach to the Hadamard Condition in Quantum Field Theory on Curved Space-Time, Comm. Math. Phys. 179 (1996), 529.

[RS75] M. Reed and B. Simon, Methods of Modern Mathematical Physics II, Academic Press, New York, 1975.

[San08] K. Sanders, Aspects of locally covariant quantum field theory, Ph.D. thesis, University of York (U.K.), 2008.

[San09] K. Sanders, On the Reeh-Schlieder property in curved spacetime, Comm. Math. Phys. 288 (2009), 271-285. 
[San10] _ Equivalence of the (generalised) Hadamard and microlocal spectrum condition for (generalised) free fields in curved spacetime, Comm. Math. Phys. 295 (2010), 485-501.

[Sch90] K. Schmüdgen, Unbounded operator algebras and representation theory, Operator theory, advances and applications, Birkhäuser, 1990.

[Sch05] J. Schlemmer, Gleichgewichtszustände auf der de Sitter-Raumzeit, Diploma Thesis, Universität Göttingen, 2005.

[Sch10] J. Schlemmer, Local Thermal Equilibrium on Cosmological Spacetimes, Ph.D. thesis, Universität Leipzig, 2010.

[Sol10] C. Solveen, Local Thermal Equilibrium in Quantum Field Theory on Flat and Curved Spacetimes, Class. Quant. Grav. 27 (2010), 235002.

[Sto09] A. Stottmeister, Zur Thermodynamik gekrümmter Raumzeiten, Diploma Thesis, Universität Göttingen, 2009.

[Str06] N. Straumann, From primordial quantum fluctuations to the anisotropies of the cosmic microwave background radiation, Ann. Phys. 15 (2006), 701-847.

[Str09] A. Strohmeier, Microlocal Analysis, Quantum Field Theory on Curved Spacetime: Concepts and Mathematical Foundations (C. Bär and K. Fredenhagen, eds.), Lecture Notes in Physics, no. 786, Springer Heidelberg Berlin, 2009.

[SV01] H. Sahlmann and R. Verch, Microlocal Spectrum Condition and Hadamard Form for Vector-Valued Quantum Fields In Curved Spacetime, Rev. Math. Phys. 13 (2001), 1203-1246.

[SV08] J. Schlemmer and R. Verch, Local Thermal Equilibrium States and Quantum Energy Inequalities, Ann. Henri Poincaré 9 (2008), 945-978.

[Uec05] M. Uecker, Lokale Gleichgewichtszustände des elektromagnetischen Feldes, Diploma Thesis, Universität Göttingen, 2005.

[Unr76] W.G. Unruh, Notes on Black Hole Evaporation, Phys. Rev. D14 (1976), 870.

[Ver01] R. Verch, A spin-statistics theorem for quantum fields on curved spacetime manifolds in a generally covariant framework, Comm. Math. Phys. 223 (2001), 261-288.

[Ver11]__ Local covariance, renormalization ambiguity, and local thermal equilibrium in cosmology, Quantum Field Theory and Gravity. Conceptual and 
mathematical advances in the search for a unified framework. (F. Finster, O. Müller, M. Nardmann, J. Tolksdorf, and E. Zeidler, eds.), Birkhäuser, 2011.

[Wal84] R.M. Wald, General Relativity, University Of Chicago Press, 1984.

[Wal94]_, Quantum Field Theory in Curved Spacetime and Black Hole Thermodynamics, University of Chicago Press, Chicago, 1994.

[Wei08] S. Weinberg, Cosmology, Oxford University Press, Oxford, 2008.

[ZMR96] D. Zubarev, V. Morozov, and G. Röpke, Statistical Mechanics of Nonequilibrium Processes, vol. 1, Akademie Verlag, Berlin, 1996. 


\section{Acknowledgements}

First, I would like to thank Prof. D. Buchholz for giving me the opportunity to work on this inspiring topic. His ideas on local thermal equilibrium form the basis of this thesis and without his constant help and encouragement this work would not have been possible. I would also like to thank Prof. K.-H. Rehren for agreeing to become part of my committee and for finding funding for me during the last part of this thesis.

I have greatly benefited from discussions with many people, but in particular I would like to mention K. Sanders, who was always glad to answer my questions on QFT in curved spacetime during his time in Göttingen. A. Stottmeister and J. Schlemmer have also been valuable partners for discussion. Their work on particular examples of KMS states in curved spacetime helped me to draw some of my conclusions in Chapter 3. R. Verch was helpful for his criticism on my results about the structure of sets of thermal observables. I would also like to thank K. Fredenhagen for pointing out to me an important reference on thermal states in Schwarzschild spacetime. N. Pinamonti provided this reference and also helped in finding an error in one of his works. I am grateful to T.-P. Hack for taking interest in my work and our discussion on LTE in cosmological spacetime. C. Fewster helped me to better understand scaling limits at an early stage of this work, while H. Bostelmann corrected a misconception of mine on operator product expansions and scaling limits. Special thanks go to J. Fish for proof-reading the whole manuscript.

Several other people have contributed in one way or the other, if only by making me feel comfortable at the institute. In no particular order: P. Martinetti, D. Cadamuro, T. Fischer, K. Daoulas, A. Braun, F. Dradi, G. Tedesco, Y. Tanimoto, L. Wallenhorst, S. Filor, M. Bischoff, J. Slowik, A. Kukhtina, C. Köhler, S. Becker, J. Queva and others.

Financial support from GrK 1493 "Mathematische Strukturen in der Modernen Quantenphysik" is gratefully acknowledged.

On a personal note, I would like to thank Maria, Charly, Gösta and Jonathan. I am privileged to call them my friends. Finally, I would like to thank my parents. Without them, I would be nowhere. 


\title{
Lebenslauf
}

\author{
Name: Christoph Solveen \\ Geboren: 25. April 1980 in Bernburg (Saale) \\ Adresse: $\quad$ Tieckweg 4, 37075 Göttingen \\ Staatsangehörigkeit: Deutsch
}

\section{Ausbildung und Beruflicher Werdegang}

$06 / 1999$

$09 / 1999-07 / 2000$

$10 / 2000-06 / 2002$

$10 / 2002-04 / 2008$

10/2005 - 06/2006 Part Three Mathematics, University of Cambridge (United Kingdom) Master of Advanced Studies in Mathematics: Merit

07/2008 - Promotion Physik an der Georg-August-Universität Göttingen

\section{Publikation}

- C. Solveen, Local Thermal Equilibrium in Quantum Field Theory on Flat and Curved Spacetimes, Class. Quant. Grav. 27235002 (2010) 